\title{
Weak LQG metrics and Liouville first passage percolation
}

\author{
Julien Dubédat ${ }^{1} \cdot$ Hugo Falconet $^{1} \cdot$ Ewain $^{\text {Gwynne }}{ }^{2}$ (D) Joshua Pfeffer ${ }^{3}$. \\ Xin Sun ${ }^{1}$
}

Received: 18 July 2019 / Revised: 7 May 2020 / Published online: 18 June 2020

(C) The Author(s) 2020

\begin{abstract}
For $\gamma \in(0,2)$, we define a weak $\gamma$-Liouville quantum gravity ( $L Q G)$ metric to be a function $h \mapsto D_{h}$ which takes in an instance of the planar Gaussian free field and outputs a metric on the plane satisfying a certain list of natural axioms. We show that these axioms are satisfied for any subsequential limits of Liouville first passage percolation. Such subsequential limits were proven to exist by Ding et al. (Tightness of Liouville first passage percolation for $\gamma \in(0,2), 2019$. ArXiv e-prints, arXiv:1904.08021). It is also known that these axioms are satisfied for the $\sqrt{8 / 3}$-LQG metric constructed by Miller and Sheffield (2013-2016). For any weak $\gamma$-LQG metric, we obtain moment bounds for diameters of sets as well as point-to-point, set-to-set, and point-to-set distances. We also show that any such metric is locally bi-Hölder continuous with respect to the Euclidean metric and compute the optimal Hölder exponents in both directions. Finally, we show that LQG geodesics cannot spend a long time near a straight line or the boundary of a metric ball. These results are used in subsequent work by Gwynne and Miller which proves that the weak $\gamma$-LQG metric is unique for each $\gamma \in(0,2)$, which in turn gives the uniqueness of the subsequential limit of Liouville first passage percolation. However, most of our results are new even in the special case when $\gamma=\sqrt{8 / 3}$
\end{abstract}

Keywords Liouville quantum gravity · Gaussian free field · LQG metric · Liouville first passage percolation

Mathematics Subject Classification 60D05 (geometric probability) · 60 G60 (random fields)

\section{Ewain Gwynne}

eg558@cam.ac.uk

Extended author information available on the last page of the article 


\section{Contents}

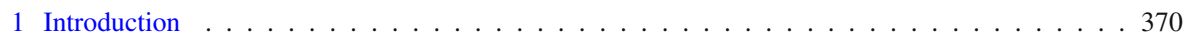

1.1 Overview . . . . . . . . . . . . . . . . . . . . 370

1.2 Weak LQG metrics and subsequential limits of LFPP . . . . . . . . . . . . . . . . . . 372

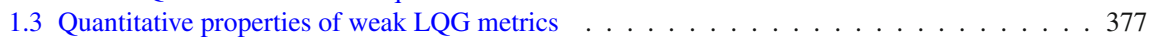

1.4 Outline . . . . . . . . . . . . . . . . . . . . . . . . . 381

1.5 Basic notation . . . . . . . . . . . . . . . . . . . . . . 381

2 Subsequential limits of LFPP are weak LQG metrics . . . . . . . . . . . . . . . . . 382

2.1 A localized version of LFPP _ . . . . . . . . . . . . . . . . . . . . . . . 382

2.2 Subsequential limits . . . . . . . . . . . . . . . . . . . . . . . . . 386

2.3 Weyl scaling . . . . . . . . . . . . . . . . . . . . . . . . . . . 391

2.4 Tightness across scales . . . . . . . . . . . . . . . . . . . . . . . . . . . 392

2.5 Locality . . . . . . . . . . . . . . . . . . . . . . . . . . . . . . . . . 394

2.6 Measurability . . . . . . . . . . . . . . . . . . . . . . . . . . . . . . . . 398

3 Proofs of quantitative properties of weak LQG metrics . . . . . . . . . . . . . . . 400

3.1 Estimate for the distance between sets . . . . . . . . . . . . . . . . . . . . 401

3.2 Asymptotics of the scaling constants . . . . . . . . . . . . . . . . . 405

3.3 Moment bound for diameters . . . . . . . . . . . . . . . . . . . . . . . 409

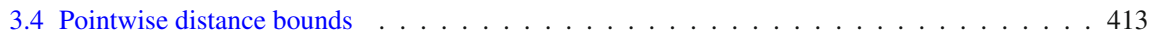

3.5 Hölder continuity . . . . . . . . . . . . . . . . . . . . . . . . . 422

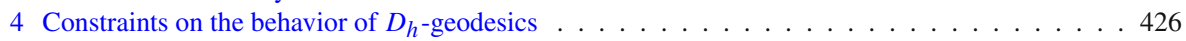

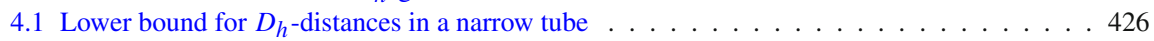

$4.2 D_{h}$-geodesics cannot trace the boundaries of $D_{h}$-metric balls $\ldots \ldots \ldots \ldots$. . . . . . . 429

References . . . . . . . . . . . . . . . . . . . . . . . . 433

\section{Introduction}

\subsection{Overview}

Let $\gamma \in(0,2)$, let $U \subset \mathbb{C}$ be open, and let $h$ be some variant of the Gaussian free field (GFF) on $U$. The $\gamma$-Liouville quantum gravity ( $L Q G)$ surface corresponding to $(U, h)$ is, heuristically speaking, the random two-dimensional Riemannian manifold with metric tensor $e^{\gamma h}\left(d x^{2}+d y^{2}\right)$, where $d x^{2}+d y^{2}$ denotes the Euclidean metric tensor. LQG surfaces are the scaling limits of various types of random planar maps: the case when $\gamma=\sqrt{8 / 3}$ corresponds to uniform random planar maps. Other values of $\gamma$ correspond to random planar maps weighted by the partition function of a statistical mechanics model on the map, e.g., the uniform spanning tree for $\gamma=\sqrt{2}$ or the critical Ising model for $\gamma=\sqrt{3}$. More generally, convergence to $\gamma$-LQG is expected if the planar map is weighted by the partition function of a critical statistical mechanics model with central charge $\mathbf{c}=25-6(2 / \gamma+\gamma / 2)^{2}$; see, e.g., [22, Section 3.1] and the references therein for further discussion.

The above definition of a $\gamma$-LQG surface does not make rigorous sense since the GFF is a random distribution, not a function. In particular, it does not have well-defined pointwise values and so cannot be exponentiated. Therefore, one needs to use various regularization procedures to make rigorous sense of LQG surfaces. For example, one can construct a random measure $\mu_{h}$ on $U$, called the $\gamma$-LQG area measure, as a limit of regularized versions of " $e^{\gamma h} d z$ ", where $d z$ denotes Lebesgue measure $[16,33,44]$. This measure can be thought of as the volume form associated with the $\gamma$-LQG surface. 
One way to construct $\mu_{h}$ is as follows. Let $p_{s}(z, w)=\frac{1}{2 \pi s} \exp \left(-\frac{|z-w|^{2}}{2 s}\right)$ be the heat kernel on $\mathbb{C}$. For $\varepsilon>0$, we define a mollified version of the GFF by

$$
h_{\varepsilon}^{*}(z):=\left(h * p_{\varepsilon^{2} / 2}\right)(z)=\int_{U} h(w) p_{\varepsilon^{2} / 2}^{U}(z, w) d w, \quad \forall z \in U,
$$

where the integral is interpreted in the sense of distributional pairing (see Remark 1.1 for some discussion on the particular choice of mollifier). One can then define the $\gamma$-LQG measure $\mu_{h}$ as the a.s. weak limit $[6,44]$

$$
\lim _{\varepsilon \rightarrow 0} \varepsilon^{\gamma^{2} / 2} e^{\gamma h_{\varepsilon}^{*}(z)} d z
$$

The LQG measure $\mu_{h}$ satisfies a conformal coordinate change formula: if $\phi: \widetilde{U} \rightarrow$ $U$ is a conformal map and

$$
\tilde{h}:=h \circ \phi+Q \log \left|\phi^{\prime}\right|, \text { where } Q=\frac{2}{\gamma}+\frac{\gamma}{2}
$$

then $\mu_{h}(A)=\mu_{\widetilde{h}}\left(\phi^{-1}(A)\right)$ for each Borel set $A \subset U$. We think of two pairs $(U, h)$ and $(\widetilde{U}, \widetilde{h})$ which are related by a conformal map as in (1.3) as being two different parametrizations of the same LQG surface. Thus the coordinate change formula for $\mu_{h}$ says that this measure depends only on the quantum surface, not on the particular choice of parametrization.

Since $\gamma$-LQG surfaces are thought of as random Riemannian manifolds, one expects that such a surface also gives rise to a random metric $D_{h}$ on $U$. Constructing such a metric is a much harder problem than constructing the measure $\mu_{h}$. Miller and Sheffield $[38,39,43]$ constructed such a metric in the special case when $\gamma=\sqrt{8 / 3}$ by using a process called quantum Loewner evolution [41] to define $\sqrt{8 / 3}$-LQG metric balls. They also showed that in this case, the metric space $\left(U, D_{h}\right)$ for certain special choices of $U$ and $D_{h}$ is isometric to a known Brownian surface-like the Brownian map [35,36] or the Brownian disk [7]. Brownian surfaces are random metric spaces which arise as the scaling limits of uniform random planar maps with respect to the Gromov-Hausdorff topology.

This paper is part of a program whose eventual goal is to construct a metric on $\gamma$-LQG for all $\gamma \in(0,2)$ as a limit of regularized metrics analogous to (1.2). These regularized metrics are called Liouville first passage percolation (LFPP). We recall the precise definition of LFPP just below. It was previously shown by Ding et al. [10] that LFPP admits non-degenerate subsequential limits in law w.r.t. the local uniform topology (i.e., the topology of uniform convergence on compact sets). The main contributions of this paper are as follows.

- Properties of subsequential limits of LFPP We prove, using a general theorem from [26], that every subsequential limit of LFPP can be realized as a measurable function of the field, so the convergence occurs in probability, not just in distribution. We also check that every subsequential limit of LFPP satisfies a certain natural list of axioms which one would expect any reasonable notion of a metric 
on $\gamma$-LQG to satisfy (see Sect. 1.2). We call a metric satisfying these axioms a weak $L Q G$ metric. A closely related list of axioms appeared previously in [37].

- Properties of weak LQG metrics We prove several quantitative properties for a general weak LQG metric. We compute the optimal Hölder exponents between the LQG metric and the Euclidean metric in both directions. We also give moment bounds for LQG diameters and for point-to-point, set-to-set, and point-to-set distances; these bounds are analogous to known moment bounds for the $\gamma$-LQG measure (see, e.g., [44]). See Sect. 1.3 for precise statements. Since our list of axioms is satisfied for the Miller-Sheffield $\sqrt{8 / 3}-\mathrm{LQG}$ metric, our results apply to this metric as well. Even in this special case, most of our results are new.

The results in this paper are used to prove further properties of weak LQG metrics (including subsequential limits of LFPP) in [23-25], eventually culminating in the proof in [25] that there is only one weak $\gamma$-LQG metric for each $\gamma \in(0,2)$, which establishes the existence and uniqueness of the $\gamma$-LQG metric for all $\gamma \in(0,2)$. However, even after this program is completed, we expect that our results will continue to be a useful tool in the study of the $\gamma$-LQG metric. For example, our estimates for the LQG metric are used in [31] to prove a version of the KPZ formula [16,34] for this metric. Moreover, as explained in Remark 1.1, our results for subsequential limits of LFPP apply to variants of LFPP defined using different continuous approximations for the GFF (other than convolution with the heat kernel) once tightness is established for these variants.

We remark that versions of some of the estimates for weak LQG metrics which are proven in this paper (including tail estimates for the distance across a rectangle, the first moment bound for diameters, and Hölder continuity) were previously proven for subsequential limits of LFPP in [10]. However, it is important to have these estimates for general weak $\gamma$-LQG metrics: indeed, such estimates will be used in [25] to show the uniqueness of the weak $\gamma$-LQG metric (which is a stronger statement than just the uniqueness of the subsequential limit for the variant of LFPP considered in [10]). Many of our estimates are also new for subsequential limits of LFPP, e.g., the optimality of the Hölder exponents in Theorem 1.7, the moment bounds in Theorems 1.8, 1.10, and 1.11, and the estimates for geodesics in Sect. 4.

Due to our axiomatic approach, our proofs do not require any outside input besides the existence of LFPP subsequential limits from [10] and a general theorem about local metrics from [26] (both of which can be taken as black boxes). To understand the paper, the reader only needs to be familiar with basic properties of the GFF, as reviewed, e.g., in [46] and the introductory sections of [40,42,47].

\subsection{Weak LQG metrics and subsequential limits of LFPP}

Let us now discuss the approximations of LQG metrics which we will be interested in. We first need to introduce an exponent which plays a fundamental role in the study of $\gamma$-LQG distances. It is shown in [12] that for each $\gamma \in(0,2)$, there is an exponent $d_{\gamma}>2$ which arises in various approximations of LQG distances. For example, for certain random planar maps in the $\gamma$-LQG universality class, a graph-distance ball of radius $r \in \mathbb{N}$ in the map typically has of order $r^{d_{\gamma}+o_{r}(1)}$ vertices. It is shown 
in [31] that $d_{\gamma}$ is the Hausdorff dimension of the $\gamma$-LQG metric. The value of $d_{\gamma}$ is not known explicitly except for $d_{\sqrt{8 / 3}}=4$, but reasonably tight upper and lower bounds are available; see [12]. We define

$$
\xi=\xi_{\gamma}:=\frac{\gamma}{d_{\gamma}}
$$

For concreteness, we will primarily focus on the whole-plane case. We say that a random distribution $h$ on $\mathbb{C}$ is a whole plane GFF plus a continuous function if there exists a coupling of $h$ with a random continuous function $f: \mathbb{C} \rightarrow \mathbb{R}$ such that the law of $h-f$ is that of a whole-plane GFF. If such a coupling exists for which $f$ is bounded, then we say that $h$ is a whole-plane GFF plus a bounded continuous function. ${ }^{1}$ Note that the whole-plane GFF is defined only modulo a global additive constant, but these definitions do not depend on the choice of additive constant.

If $h$ is a whole-plane GFF, or more generally a whole-plane GFF plus a bounded continuous function, we define the mollified GFF $h_{\varepsilon}^{*}(z)$ for $\varepsilon>0$ and $z \in \mathbb{C}$ as in (1.1). For $z, w \in \mathbb{C}$ and $\varepsilon>0$, we define the $\varepsilon$-LFPP metric by ${ }^{2}$

$$
D_{h}^{\varepsilon}(z, w):=\inf _{P: z \rightarrow w} \int_{0}^{1} e^{\xi h_{\varepsilon}^{*}(P(t))}\left|P^{\prime}(t)\right| d t
$$

where the infimum is over all piecewise continuously differentiable paths from $z$ to $w$. One should think of LFPP as the metric analog of the approximations of the LQG measure in (1.2).

Remark 1.1 The reason why we define LFPP using $h_{\varepsilon}^{*}$ instead of some other continuous approximation of the GFF is that this is the approximation for which tightness is proven in [10]. If we had a tightness result similar to those in [10] for LFPP defined using a different approximation (such as the circle average process of [16, Section 3.1] or the convolution of $h$ with $\varepsilon^{-1} \phi(|z-w| / \sqrt{\varepsilon})$, where $\phi$ is a continuous non-negative radially symmetric function with total integral one), then similar arguments to those in Sect. 2 would show that the subsequential limits are also weak LQG metrics. Together with the uniqueness of weak LQG metrics proven in [25], this means that in order to show that such approximations converge to the $\gamma$-LQG metric one only needs to prove tightness.

For $\varepsilon>0$, let $\mathfrak{a}_{\varepsilon}$ be the median of the $D_{h}^{\varepsilon}$-distance between the left and right boundaries of the unit square along paths which stay in the unit square. It follows from results in [10] (see Lemma 2.5) that the laws of the metrics $\left\{\mathfrak{a}_{\varepsilon}^{-1} D_{h}^{\varepsilon}\right\}_{\varepsilon>0}$ are tight with respect to the local uniform topology on $\mathbb{C} \times \mathbb{C}$ and every subsequential limit induces the Euclidean topology on $\mathbb{C}$.

\footnotetext{
1 The reason why we sometimes restrict to bounded continuous functions is that it ensures that the convolution with the whole-plane heat kernel is finite (so $D_{h}^{\varepsilon}$ is defined) and it makes parts of the proof of Theorem 1.2 simpler.

2 The intuitive reason why we look at $e^{\xi h_{\varepsilon}^{*}(z)}$ instead of $e^{\gamma h_{\varepsilon}^{*}(z)}$ to define the metric is as follows. By (1.2), we can scale LQG areas by a factor of $C>0$ by adding $\gamma^{-1} \log C$ to the field. By (1.5), this results in scaling distances by $C^{\xi / \gamma}=C^{1 / d}$, which is consistent with the fact that the "dimension" should be the exponent relating the scaling of areas and distances.
} 
Building on this, we will prove that in fact the metrics $\mathfrak{a}_{\varepsilon}^{-1} D_{h}^{\varepsilon}$ admit subsequential limits in probability and that every subsequential limit satisfies a certain natural list of axioms. To state these axioms, we need some preliminary definitions. Let $(X, D)$ be a metric space.

For a curve $P:[a, b] \rightarrow X$, the $D$-length of $P$ is defined by

$$
\operatorname{len}(P ; D):=\sup _{T} \sum_{i=1}^{\# T} D\left(P\left(t_{i}\right), P\left(t_{i-1}\right)\right)
$$

where the supremum is over all partitions $T: a=t_{0}<\cdots<t_{\# T}=b$ of $[a, b]$. Note that the $D$-length of a curve may be infinite.

For $Y \subset X$, the internal metric of $D$ on $Y$ is defined by

$$
D(x, y ; Y):=\inf _{P \subset Y} \operatorname{len}(P ; D), \quad \forall x, y \in Y
$$

where the infimum is over all paths $P$ in $Y$ from $x$ to $y$. Then $D(\cdot, \cdot ; Y)$ is a metric on $Y$, except that it is allowed to take infinite values.

We say that $(X, D)$ is a length space if for each $x, y \in X$ and each $\varepsilon>0$, there exists a curve of $D$-length at most $D(x, y)+\varepsilon$ from $x$ to $y$.

A continuous metric on a domain $U \subset \mathbb{C}$ is a metric $D$ on $U$ which induces the Euclidean topology on $U$, i.e., the identity map $(U,|\cdot|) \rightarrow(U, D)$ is a homeomorphism. We equip the space of continuous metrics on $U$ with the local uniform topology for functions from $U \times U$ to $[0, \infty)$ and the associated Borel $\sigma$-algebra. We allow a continuous metric to have $D(u, v)=\infty$ if $u$ and $v$ are in different connected components of $U$. In this case, in order to have $D^{n} \rightarrow D$ w.r.t. the local uniform topology we require that for large enough $n, D^{n}(u, v)=\infty$ if and only if $D(u, v)=\infty$.

Let $\mathcal{D}^{\prime}(\mathbb{C})$ be the space of distributions (generalized functions) on $\mathbb{C}$, equipped with the usual weak topology. For $\gamma \in(0,2)$, a weak $\gamma-L Q G$ metric is a measurable function $h \mapsto D_{h}$ from $\mathcal{D}^{\prime}(\mathbb{C})$ to the space of continuous metrics on $\mathbb{C}$ such that the following is true whenever $h$ is a whole-plane GFF plus a continuous function.

I. Length space Almost surely, $\left(\mathbb{C}, D_{h}\right)$ is a length space, i.e., the $D_{h}$-distance between any two points of $\mathbb{C}$ is the infimum of the $D_{h}$-lengths of $D_{h}$-continuous paths (equivalently, Euclidean continuous paths) between the two points.

II. Locality Let $U \subset \mathbb{C}$ be a deterministic open set. The $D_{h}$-internal metric $D_{h}(\cdot, \cdot ; U)$ is determined a.s. by $\left.h\right|_{U}$.

III. Weyl scaling Let $\xi$ be as in (1.4) and for each continuous function $f: \mathbb{C} \rightarrow \mathbb{R}$, define

$$
\left(e^{\xi f} \cdot D_{h}\right)(z, w):=\inf _{P: z \rightarrow w} \int_{0}^{\operatorname{len}\left(P ; D_{h}\right)} e^{\xi f(P(t))} d t, \quad \forall z, w \in \mathbb{C},
$$

where the infimum is over all continuous paths from $z$ to $w$ parametrized by $D_{h^{-}}$ length. Then a.s. $e^{\xi f} \cdot D_{h}=D_{h+f}$ for every continuous function $f: \mathbb{C} \rightarrow \mathbb{R}$.

IV. Translation invariance For each deterministic point $z \in \mathbb{C}$, a.s. $D_{h(\cdot+z)}=D_{h}(\cdot+$ $z, \cdot+z)$. 
V. Tightness across scales Suppose that $h$ is a whole-plane GFF and let $\left\{h_{r}(z)\right\}_{r>0, z \in \mathbb{C}}$ be its circle average process. For each $r>0$, there is a deterministic constant $\mathfrak{c}_{r}>0$ such that the set of laws of the metrics $\mathfrak{c}_{r}^{-1} e^{-\xi h_{r}(0)} D_{h}(r \cdot, r \cdot)$ for $r>0$ is tight (w.r.t. the local uniform topology). Furthermore, the closure of this set of laws w.r.t. the Prokhorov topology on continuous functions $\mathbb{C} \times \mathbb{C} \rightarrow[0, \infty)$ is contained in the set of laws on continuous metrics on $\mathbb{C}$ (i.e., every subsequential limit of the laws of the metrics $\mathfrak{c}_{r}^{-1} e^{-\xi h_{r}(0)} D_{h}(r \cdot r \cdot)$ is supported on metrics which induce the Euclidean topology on $\mathbb{C}$ ). Finally, there exists $\Lambda>1$ such that for each $\delta \in(0,1)$,

$$
\Lambda^{-1} \delta^{\Lambda} \leq \frac{\mathfrak{c}_{\delta r}}{\mathfrak{c}_{r}} \leq \Lambda \delta^{-\Lambda}, \quad \forall r>0
$$

We emphasize that the definition of a weak $\gamma$-LQG metric depends on $\gamma$ only via the parameter $\xi$ in Axiom III. We will therefore sometimes say that a metric satisfying the above axioms is a weak $L Q G$ metric with parameter $\xi$.

It is easy to see, at least heuristically, why Axioms I through V should be satisfied for subsequential limits of LFPP, although there is some subtlety involved in checking these axioms rigorously. The first main result of this paper is the following statement, whose proof builds on results from [10,26].

Theorem 1.2 Let $\gamma \in(0,2)$. For every sequence of $\varepsilon$ 's tending to zero, there is a weak $\gamma$-LQG metric D and a subsequence $\left\{\varepsilon_{n}\right\}_{n \in \mathbb{N}}$ for which the following is true. Let $h$ be a whole-plane GFF, or more generally a whole-plane GFF plus a bounded continuous function. Then the re-scaled LFPP metrics $\mathfrak{a}_{\varepsilon_{n}}^{-1} D_{h}^{\varepsilon_{n}}$ from (1.5) converge in probability to $D_{h}$.

We will explain why we get convergence in probability, instead of just in law, in Theorem 1.2 just below. Let us first discuss the axioms for a weak LQG metric. Axioms I through IV are natural from the perspective that $\gamma$-LQG is a "random two-dimensional Riemannian manifold" obtained by exponentiating $h$. Axiom V is a substitute for exact scale invariance of the metric. To explain this, it is expected (and will be proven in $[24,25]$ ) that the $\gamma$-LQG metric, like the $\gamma$-LQG measure, is invariant under coordinate changes of the form (1.3). In particular, it should be the case that for any $a \in \mathbb{C} \backslash\{0\}$, a.s.

$$
D_{h}(a \cdot, a \cdot)=D_{h(a \cdot)+Q \log |a|}(\cdot, \cdot), \quad \text { for } \quad Q=\frac{2}{\gamma}+\frac{\gamma}{2} .
$$

Under Axiom III, the formula (1.9) together with the scale invariance of the law of $h$, modulo an additive constant, implies Axiom $\mathrm{V}$ with $\mathfrak{c}_{r}=r^{\xi Q}$. We define a strong $L Q G$ metric to be a mapping $h \mapsto D_{h}$ which satisfies Axioms I through IV as well as (1.9).

A similar definition of a strong LQG metric has appeared in earlier literature. Indeed, the paper [37] proved several properties of geodesics for any metric associated with $\gamma$-LQG which satisfies a similar list of axioms to the ones in our definition of a 
strong LQG metric; however, at that point such a metric had only been constructed for $\gamma=\sqrt{8 / 3}$. $^{3}$

It far from obvious that subsequential limits of LFPP satisfy (1.9). The reason for this is that scaling space results in scaling the value of $\varepsilon$ in (1.5), which in turn changes the subsequence which we are working with. It will eventually be proven in [25] that every weak LQG metric satisfies (1.9), i.e., every weak LQG metric is a strong LQG metric, but the proof requires all of the results of the present paper as well as those of $[23,26]$.

Nevertheless, Axiom V can be used in place of (1.9) in many situations. Basically, this axiom allows us to compare distance quantities at the same Euclidean scale. For example, Axiom $\mathrm{V}$ implies that if $U \subset \mathbb{C}$ is open and $K \subset U$ is compact, then the laws of

$$
\left(\mathfrak{c}_{r}^{-1} e^{-\xi h_{r}(0)} D_{h}(r K, r \partial U)\right)^{-1} \text { and } \mathfrak{c}_{r}^{-1} e^{-\xi h_{r}(0)} \sup _{u, v \in r K} D_{h}(u, v ; r U)
$$

as $r$ varies are tight.

Part of the proof of Theorem 1.2 is to show that for any joint subsequential limit ( $h, D_{h}$ ) of the laws of the pairs $\left(h, \mathfrak{a}_{\varepsilon}^{-1} D_{h}^{\varepsilon}\right)$, the limiting metric $D_{h}$ is a measurable function of $h$. This is not obvious since convergence in law does not in general preserve measurability. In our setting, we will prove that $D_{h}$ is determined by $h$ by checking the conditions of [26, Corollary 1.8], which gives a list of conditions under which a random metric coupled with the GFF is determined by the GFF. The reason why we have convergence in probability, instead of convergence in law, in Theorem 1.2 is the following elementary probabilistic lemma (see e.g. [47, Lemma 4.5]). ${ }^{4}$

Lemma 1.3 Let $\left(\Omega_{1}, d_{1}\right)$ and $\left(\Omega_{2}, d_{2}\right)$ be complete separable metric spaces. Let $X$ be a random variable taking values in $\Omega_{1}$ and let $\left\{Y^{n}\right\}_{n \in \mathbb{N}}$ and $Y$ be random variables taking values in $\Omega_{2}$, all defined on the same probability space, such that $\left(X, Y^{n}\right) \rightarrow$ $(X, Y)$ in law. If $Y$ is a.s. determined by $X$, then $Y^{n} \rightarrow Y$ in probability.

Theorem 1.2 will be proven in Sect. 2. Once this is done, throughout the rest of the paper we will only ever work with a weak $\gamma$-LQG metric-we will not need to make explicit reference to LFPP. An important advantage of this approach is that the Miller-Sheffield $\sqrt{8 / 3}-\mathrm{LQG}$ metric from $[38,39,43]$ is known to satisfy the axioms

\footnotetext{
3 Although the axioms in [37] are formulated in a slightly different way from our axioms for a strong LQG metric, it can be proven, with some work, that the two notions are equivalent. The analog of Axiom II in [37], which asserts that metric balls are local sets, is proven to be equivalent to our Axiom II in [26, Lemma 2.2]. The analog of Axiom III in [37] is stated only for constant functions, but it is easy to check that this axiom implies Axiom III. For example, this is explained in [29, Section 2.4] in the special case when $\gamma=\sqrt{8 / 3}$, and the same argument works for general $\gamma \in(0,2)$. In [37, Assumption 1.1], the authors allow for fields on any open domain in $\mathbb{C}$ and assume that the metric satisfies a LQG coordinate change formula for general conformal maps, not just complex affine maps. It is shown in [24] that a strong LQG metric in the sense of this paper gives rise to a metric associated with a GFF on any proper sub-domain of $\mathbb{C}$ which satisfies the LQG coordinate change formula for general conformal maps.

4 Since the space of continuous metrics is not complete w.r.t. any natural choice of metric which induces the local uniform topology, we apply the lemma with $\left(\Omega_{2}, d_{2}\right)$ equal to the larger space of continuous functions $\mathbb{C} \times \mathbb{C} \rightarrow[0, \infty)$ equipped with the local uniform topology, which is completely metrizable.
} 
for a weak $\sqrt{8 / 3}-$ LQG metric. See $[29$, Section 2.4] for a careful explanation of why this is the case. Note that [29, Section 2.4] checks the coordinate change relation (1.9) for the Miller-Sheffield metric which (as discussed above) implies Axiom V. Hence all of our results for weak $\gamma$-LQG metrics apply to both this $\sqrt{8 / 3}$-LQG metric and to subsequential limits of LFPP. ${ }^{5}$

Remark 1.4 (Liouville graph distance) Besides LFPP, there is another natural scheme for approximating LQG metrics called Liouville graph distance (LGD). The $\varepsilon$-LGD distance between two points in $\mathbb{C}$ is defined to be the minimum number of Euclidean balls with LQG mass $\varepsilon$ whose union contains a path between the two points. It has been proven in [9] that for each $\gamma \in(0,2)$, the $\varepsilon$-LGD metric, appropriately renormalized, admits subsequential limiting metrics as $\varepsilon \rightarrow 0$ which induce the Euclidean topology. In the contrast to LFPP, for subsequential limits of LGD the coordinate change relation (1.9) is easy to verify but Weyl scaling (Axiom III) appears to be very difficult to verify, so these subsequential limits are not known to be weak LQG metrics in the sense of this paper. It is still an open problem to establish uniqueness of the scaling limit for LGD. Similar considerations apply to variants of LGD defined using embedded planar maps (such as maps constructed from LQG square subdivision [16,19] or mated-CRT maps $[21,27]$ ) instead of Euclidean balls, although for these variants tightness has not been checked.

\subsection{Quantitative properties of weak LQG metrics}

In what follows, we assume that $D$ is a weak $\gamma$-LQG metric and $h$ is a whole-plane GFF. Perhaps surprisingly, the axioms for a weak LQG metric imply much sharper bounds on the scaling constants $\mathfrak{c}_{r}$ than (1.8).

Theorem 1.5 Let $\xi$ be as in (1.4) and let $Q=2 / \gamma+\gamma / 2$. Then for $r>0$, the scaling constants satisfy

$$
\frac{\mathfrak{c}_{\delta r}}{\mathfrak{c}_{r}}=\delta^{\xi Q+o_{\delta}(1)} \quad \text { as } \delta \rightarrow 0
$$

at a rate which is uniform over all $r>0$.

The definition of a weak LQG metric uses only the parameter $\xi$. Theorem 1.5 connects this definition to the coordinate change parameter $Q$. This will be important for the proof in [25] that any weak LQG metric satisfies the coordinate change formula (1.9). Theorem 1.5 will be proven in Sect. 3.2 by comparing $D_{h}$-distances to LFPP distances and using the fact that the $\delta$-LFPP distance between two fixed points is typically of order $\delta^{1-\xi Q+o_{\delta}(1)}$ [12, Theorem 1.5] [for convenience, for this argument we will work with a variant of LFPP which is defined in a slightly different manner than the version in (1.5)].

\footnotetext{
5 The uniqueness of the weak LQG metric proven in [25] implies that the Miller-Sheffield $\sqrt{8 / 3}$-LQG metric is the limit of LFPP for $\gamma=\sqrt{8 / 3}$.
} 
Remark 1.6 Theorem 1.5 gives a proof purely in the continuum that the exponent $d_{\sqrt{8 / 3}}$ of $[12,18]$ is equal to 4 . Previously, this was proven in [12] (building on [20]) using the known ball volume growth exponent for random triangulations [1]. To see why Theorem 1.5 implies that $d_{\sqrt{8 / 3}}=4$, we observe that the $\sqrt{8 / 3}$-LQG metric of $[38,39,43]$ satisfies the axioms for a weak LQG metric with parameter $\xi=1 / \sqrt{6}$. Moreover, by the LQG coordinate change formula for the $\sqrt{8 / 3}-\mathrm{LQG}$ metric, Axiom V holds for this metric with with $\mathfrak{c}_{r}=r^{5 / 6}$. Theorem 1.5 therefore implies that if $\gamma \in$ $(0,2)$ is chosen so that $\gamma / d_{\gamma}=1 / \sqrt{6}$, then the associated parameter $Q=2 / \gamma+\gamma / 2$ satisfies $Q / \sqrt{6}=5 / 6$, i.e., $Q=5 / \sqrt{6}$ which is equivalent to $\gamma=\sqrt{8 / 3}$. Hence $\gamma / d_{\gamma}=1 / \sqrt{6}$ when $\gamma=\sqrt{8 / 3}$, so $d_{\sqrt{8 / 3}}=4$.

Our next main result gives the optimal Hölder exponents for $D_{h}$ with respect to the Euclidean metric.

Theorem 1.7 (Optimal Hölder exponents) Let $U \subset \mathbb{C}$ be open and bounded. Almost surely, the identity map from $U$, equipped with the Euclidean metric, to $\left(U, D_{h}\right)$ is locally Hölder continuous with any exponent smaller than $\xi(Q-2)$ and is not locally Hölder continuous with any exponent larger than $\xi(Q-2)$. Furthermore, the inverse of this map is a.s. locally Hölder continuous with any exponent smaller than $\xi^{-1}(Q+2)^{-1}$ and is not locally Hölder continuous with any exponent larger than $\xi^{-1}(Q+2)^{-1}$.

For $\gamma=\sqrt{8 / 3}$, one has $\xi=1 / \sqrt{6}$ and $Q=5 / \sqrt{6}$, so the optimal Hölder exponents are given by

$\xi(Q-2)=\frac{1}{6}(5-2 \sqrt{6}) \approx 0.0168$ and $\xi^{-1}(Q+2)^{-1}=30-12 \sqrt{6} \approx 0.6061$.

The intuitive reason why Theorem 1.7 is true is as follows. If $z$ is an $\alpha$-thick point for $h$, i.e., the circle average satisfies $h_{\varepsilon}(z)=\left(\alpha+o_{\varepsilon}(1)\right) \log \varepsilon^{-1}$ as $\varepsilon \rightarrow 0$, then we can show that the $D_{h}$-distance from $z$ to $\partial B_{\varepsilon}(z)$ behaves like $\varepsilon^{\xi(Q-\alpha)+o_{\varepsilon}(1)}$ as $\varepsilon \rightarrow 0$. Indeed, this is an easy consequence of the estimates in Sect. 3.4. Almost surely, $\alpha$-thick points exist for $\alpha \in(-2,2)$ but not for $|\alpha|>2$ [32].

We next state some basic moment estimates for distances which are metric analogues of the well-known fact that the $\gamma$-LQG measure has finite moments of all orders in $\left(-\infty, 4 / \gamma^{2}\right)$ [44, Theorems 2.11 and 2.12].

Theorem 1.8 (Moment bounds for diameters) Let $U \subset \mathbb{C}$ be open and let $K \subset U$ be a compact connected set with more than one point. Then the $U$-internal diameter of $K$ satisfies

$$
\mathbb{E}\left[\left(\sup _{z, w \in K} D_{h}(z, w ; U)\right)^{p}\right]<\infty, \quad \forall p \in\left(-\infty, \frac{4 d_{\gamma}}{\gamma^{2}}\right)
$$

For $\gamma=\sqrt{8 / 3}$, we get finite moments up to order 6 . We also have the following bound for distances between sets. In this case, we get finite moments of all orders. 
Theorem 1.9 (Distance between sets) Let $U \subset \mathbb{C}$ be an open set (possibly all of $\mathbb{C}$ ) and let $K_{1}, K_{2} \subset U$ be connected, disjoint compact sets which are not singletons. Then

$$
\mathbb{E}\left[\left(D_{h}\left(K_{1}, K_{2} ; U\right)\right)^{p}\right]<\infty, \quad \forall p \in \mathbb{R}
$$

The results of [10] show that if $D_{h}$ is a subsequential scaling limit of the LFPP metrics (1.5), then one has the following slightly stronger version of Theorem 1.9:

$$
\mathbb{P}\left[A^{-1} \leq \mathfrak{a}_{\varepsilon}^{-1} D_{h}^{\varepsilon}\left(K_{1}, K_{2} ; U\right) \leq A\right] \geq 1-c_{0} e^{-c_{1}(\log A)^{2} / \log \log A}, \quad \forall A>2 e^{e}
$$

for constants $c_{0}, c_{1}>0$ allowed to depend on $K_{1}, K_{2}, U$. A posteriori, one gets (1.15) for every weak LQG metric since [25] proves that the weak LQG metric is unique for each $\gamma \in(0,2)$, so in particular it is the limit of LFPP.

We now turn our attention to point-to-point distances. These estimates also work if we allow the field to have a log singularity. To make sense of the metric in this case, we note that since $\log |\cdot|$ is continuous away from 0 , we can define $D_{h-\alpha \log |\cdot|}$ as a continuous length metric on $\mathbb{C} \backslash\{0\}$ by $D_{h-\alpha \log |\cdot|}=|\cdot|^{-\alpha \xi} \cdot D_{h}$, in the notation (1.7). We can then extend $D_{h-\alpha \log |\cdot|}$ to a metric defined on all of $\mathbb{C}$ which is allowed to take

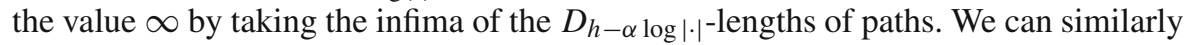
define the metric associated with fields with two or more log singularities.

Theorem 1.10 (Distance from a point to a circle) Let $\alpha \in \mathbb{R}$ and let $h^{\alpha}:=h-\alpha \log |\cdot|$. If $\alpha \in(-\infty, Q)$, then

$$
\mathbb{E}\left[\left(D_{h^{\alpha}}(0, \partial \mathbb{D})\right)^{p}\right]<\infty, \quad \forall p \in\left(-\infty, \frac{2 d_{\gamma}}{\gamma}(Q-\alpha)\right) .
$$

If $\alpha>Q$, then a.s. $D_{h^{\alpha}}(0, z)=\infty$ for every $z \in \mathbb{C} \backslash\{0\}$.

For example, if $\gamma=\sqrt{8 / 3}$ and $\alpha=0$, we get finite moments up to order 10. If instead $\gamma=\sqrt{8 / 3}$ and $\alpha=\gamma$ (which corresponds to the case when 0 is a "quantum typical" point, see, e.g., [16, Proposition 3.4]) we only get finite moments up to order 2 . In the critical case when $\alpha=Q$, our estimates at this point are not sufficiently sharp to determine whether $D_{h} Q(0, \partial \mathbb{D})$ is finite. However, once we know that every weak LQG metric is a strong LQG metric (which is proven in [25]) it is not hard to check that a.s. $D_{h Q}(0, z)=\infty$ for every $z \in \mathbb{C} \backslash\{0\}$. Similar comments apply in the case when $\alpha=Q$ or $\beta=Q$ in Theorem 1.11 just below.

Theorem 1.11 (Distance between two points) Let $\alpha, \beta \in \mathbb{R}$, let $z, w \in \mathbb{C}$ be distinct, and let $h^{\alpha, \beta}:=h-\alpha \log |\cdot-z|-\beta \log |\cdot-w|$. If $\alpha, \beta \in(-\infty, Q)$, then

$$
\mathbb{E}\left[\left(D_{h^{\alpha}}\left(z, w ; B_{4|z-w|}(z)\right)\right)^{p}\right]<\infty, \quad \forall p \in\left(-\infty, \frac{2 d_{\gamma}}{\gamma}(Q-\max \{\alpha, \beta\})\right) .
$$


If either $\alpha>Q$ or $\beta>Q$, then a.s. $D_{h^{\alpha, \beta}}(z, w)=\infty$.

As applications of our main results, in Sect. 4 we will also prove some estimates which constrain the behavior of $D_{h}$-geodesics and which will be important in [25]. To be more precise, the first main estimate of Sect. 4 is Proposition 4.1, which gives an upper bound for the amount of time that a $D_{h}$-geodesic can spend in a small neighborhood of a line segment or a circular arc. Intuitively, one expects that this amount of time is small since LQG geodesics should be fractal and hence should look very different from smooth curves. The particular bound given in Proposition 4.1 is used in [25, Section 3] to prevent a geodesic from spending a long time in an annulus with a small aspect ratio; and in [25, Section 5] in order to force a geodesic to enter a "good" region of the plane in which certain distance bounds hold.

The other main estimate in Sect. 4 is Proposition 4.3, which is an upper bound for how much time an LQG geodesic can spend near the boundary of an LQG metric ball centered at its starting point. Intuitively, this amount of time should be small since if $P$ is a $D_{h}$-geodesic, then $D_{h}(P(0), P(t))=t$ but $D_{h}(P(0), \cdot)$ is constant on the boundary of a $D_{h}$-ball centered at $P(0)$. The bound given in Proposition 4.3 is used in [25, Lemma 4.7].

Remark 1.12 (The case when $\xi>2 / \mathbf{d}_{2}$ ) Throughout this paper, we focus on the case of weak $\gamma$-LQG metrics. Since $\gamma \mapsto \gamma / d_{\gamma}$ is increasing [12, Proposition 1.7], weak $\gamma$-LQG metrics have parameter $\xi \in\left(0,2 / d_{2}\right)$ (here, $\left.d_{2}:=\lim _{\gamma \rightarrow 2^{-}} d_{\gamma}\right)$. It is natural to wonder whether one can say anything about weak LQG metrics which satisfy the same axioms but with a parameter $\xi \geq 2 / d_{2}$. In the critical case when $\xi=2 / d_{2}$ (i.e., $\gamma=2$ ), we expect that a weak LQG metric still exists and is the scaling limit of LFPP with parameter $2 / d_{2}$. This metric should be the $\gamma$-LQG metric with $\gamma=2$ (the $\gamma=2$ metric should also be the limit as $\gamma \nearrow 2$ of the $\gamma$-LQG metrics, appropriately renormalized). We expect that all of the theorem statements in this section still hold for $\xi=2 / d_{2}$, except that the metric $D_{h}$ is not Hölder continuous w.r.t. the Euclidean metric.

For $\xi>2 / d_{2}$, we do not expect that any weak LQG metrics with parameter $\xi$ exist. However, there should be metrics which satisfy a similar list of properties except that such metrics no longer induce the Euclidean topology. Instead, there should be an uncountable, dense set of points $z \in \mathbb{C}$ such that $D_{h}(z, w)=\infty$ for every $w \in \mathbb{C} \backslash\{z\}$. More precisely, let $\lambda(\xi)$ be the exponent for the typical LFPP distance between the left and right sides of $[0,1]^{2}$ and let $Q(\xi)=(1-\lambda(\xi)) / \xi$. By [12, Theorem 1.5], $Q\left(\gamma / d_{\gamma}\right)=2 / \gamma+\gamma / 2>2$. By [30, Lemma 4.1] and [14, Theorem 1.1], $Q(\xi) \in(0,2)$ for $\xi>2 / d_{2}$. For $\xi>2 / d_{2}$, the points $z \in \mathbb{C}$ which lie at infinite $D_{h}$-distance from every other point should correspond to so-called thick points of $h$ (as defined in [32]) with thickness $\alpha>Q$.

It is shown in [13] that LFPP with parameter $\xi>2 / d_{2}$ admits subsequential scaling limits in law w.r.t. the topology on lower semicontinuous functions. We expect that the subsequential limit is unique, satisfies the properties discussed in the preceding paragraph, and is related to LQG with matter central charge $\mathbf{c} \in(1,25)$ (LQG with $\gamma \in(0,2]$ corresponds to $\mathbf{c} \in(-\infty, 1])$. In particular, with $Q(\xi)$ as above, the central charge should be related to $\xi$ by $\mathbf{c}=25-6 Q(\xi)^{2}$. See $[3,13,14,19,30]$ for 
further discussion of this extended phase of LQG and some justification for the above predictions.

\subsection{Outline}

In Sect. 2, we prove Theorem 1.2, which says that subsequential limits of LFPP are weak $\gamma$-LQG metrics, taking [10] as a starting point. Throughout the rest of the paper, we work with an arbitrary weak $\gamma$-LQG metric (not necessarily assumed to arise as a subsequential limit of LFPP). Section 3 contains the proofs of the results stated in Sect. 1.3. In fact, for most of these results, we will prove more quantitative versions which are required to be uniform over all Euclidean scales. At this point, these statements are not implied by the statements in Sect. 1.3 since we are working with a weak $\gamma$-LQG metric, which is only known to be "tight across scales" (Axiom V) instead of exactly scale invariant.

The first result that we prove for a weak $\gamma$-LQG metric is the estimate for the distance between two sets from Theorem 1.9; this is the content of Sect. 3.1. In Sect. 3.2, we use this estimate to relate $D_{h}$-distances to LFPP distances and thereby prove Theorem 1.5. Once Theorem 1.5 is established, we have some ability to compare $D_{h}$-distances at different Euclidean scales. This allows us to prove the moment estimate (1.13) of Theorem 1.8 in Sect. 3.3 as well as the moment estimates of Theorems 1.10 and 1.11 in Sect. 3.4. Using these moment estimates, we then prove Theorem 1.7 in Sect. 3.5.

In Sect. 4, we apply the estimates of Sect. 1.3 to prove some bounds for $D_{h^{-}}$ geodesics.

\subsection{Basic notation}

We write $\mathbb{N}=\{1,2,3, \ldots\}$ and $\mathbb{N}_{0}=\mathbb{N} \cup\{0\}$.

For $a<b$, we define the discrete interval $[a, b]_{\mathbb{Z}}:=[a, b] \cap \mathbb{Z}$.

If $f:(0, \infty) \rightarrow \mathbb{R}$ and $g:(0, \infty) \rightarrow(0, \infty)$, we say that $f(\varepsilon)=O_{\varepsilon}(g(\varepsilon))$ (resp. $\left.f(\varepsilon)=o_{\varepsilon}(g(\varepsilon))\right)$ as $\varepsilon \rightarrow 0$ if $f(\varepsilon) / g(\varepsilon)$ remains bounded (resp. tends to zero) as $\varepsilon \rightarrow 0$. We similarly define $O(\cdot)$ and $o(\cdot)$ errors as a parameter goes to infinity.

If $f, g:(0, \infty) \rightarrow[0, \infty)$, we say that $f(\varepsilon) \preceq g(\varepsilon)$ if there is a constant $C>0$ (independent from $\varepsilon$ and possibly from other parameters of interest) such that $f(\varepsilon) \leq$ $C g(\varepsilon)$. We write $f(\varepsilon) \asymp g(\varepsilon)$ if $f(\varepsilon) \preceq g(\varepsilon)$ and $g(\varepsilon) \preceq f(\varepsilon)$.

Let $\left\{E^{\varepsilon}\right\}_{\varepsilon>0}$ be a one-parameter family of events. We say that $E^{\varepsilon}$ occurs with

- polynomially high probability as $\varepsilon \rightarrow 0$ if there is a $p>0$ (independent from $\varepsilon$ and possibly from other parameters of interest) such that $\mathbb{P}\left[E^{\varepsilon}\right] \geq 1-O_{\varepsilon}\left(\varepsilon^{p}\right)$.

- superpolynomially high probability as $\varepsilon \rightarrow 0$ if $\mathbb{P}\left[E^{\varepsilon}\right] \geq 1-O_{\varepsilon}\left(\varepsilon^{p}\right)$ for every $p>0$.

We similarly define events which occur with polynomially or superpolynomially high probability as a parameter tends to $\infty$.

We will often specify any requirements on the dependencies on rates of convergence in $O(\cdot)$ and $o(\cdot)$ errors, implicit constants in $\preceq$, etc., in the statements of 
lemmas/propositions/theorems, in which case we implicitly require that errors, implicit constants, etc., appearing in the proof satisfy the same dependencies.

For $z \in \mathbb{C}$ and $r>0$, we write $B_{r}(z)$ for the Euclidean ball of radius $r$ centered at $z$. We also define the open annulus

$$
\mathrm{A}_{r_{1}, r_{2}}(z):=B_{r_{2}}(z) \backslash \overline{B_{r_{1}}(z)}, \quad \forall 0<r_{r}<r_{2}<\infty .
$$

We write $\mathbb{S}=(0,1)^{2}$ for the open Euclidean unit square.

\section{Subsequential limits of LFPP are weak LQG metrics}

The goal of this section is to deduce Theorem 1.2 from the tightness result of [10]. We start in Sect. 2.1 by introducing a "localized" variant of LFPP, defined using the convolution of $h$ with a truncated version of the heat kernel, which (unlike the $\varepsilon$-LFPP metric $D_{h}^{\varepsilon}$ defined in (1.5)) depends locally on $h$. We then show that this localized variant of LFPP is a good approximation for $D_{h}^{\varepsilon}$ (Lemma 2.1). In Sect. 2.2, we explain why the results of [10] imply that the re-scaled LFPP metrics $\mathfrak{a}_{\varepsilon}^{-1} D_{h}^{\varepsilon}$ as well as the associated internal metrics on certain domains in $\mathbb{C}$ are tight w.r.t. the local uniform topology and that every subsequential limit is a continuous length metric on $\mathbb{C}$. In Sections 2.3, 2.4, and 2.5, respectively, we will prove versions of Weyl scaling, tightness across scales, and locality for the subsequential limits (i.e., Axioms III, V, and II). In Sect. 2.6, we use a theorem from [26] to show that subsequential limits of LFPP can be realized as measurable functions of $h$. We then conclude the proof of Theorem 1.2.

Throughout this section, we will frequently need to switch between working with a whole-plane GFF and working with a whole-plane GFF plus a continuous function. As such, we will always write $h$ for a whole-plane GFF (with some choice of additive constant, specified as needed) and $\mathrm{h}$ for a whole-plane GFF plus a continuous function (usually, this will be a whole-plane GFF plus a bounded continuous function). Note that this differs from the convention elsewhere in the paper, where $h$ is sometimes used to denote a whole-plane GFF plus a continuous function.

\subsection{A localized version of LFPP}

Let $\mathrm{h}$ be a whole-plane GFF plus a bounded continuous function. The mollified field $\mathrm{h}_{\varepsilon}^{*}(z)$ of $(1.1)$ does not depend on $\mathrm{h}$ in a local manner, and hence $D_{\mathrm{h}}^{\varepsilon}$-distances do not depend on $\mathrm{h}$ in a local manner. However, as $\varepsilon \rightarrow 0$ the heat kernel $p_{\varepsilon^{2} / 2}(z, w)$ concentrates around the diagonal, so we expect that $h_{\varepsilon}^{*}(z)$ "almost" depends locally on $\mathrm{h}$ when $\varepsilon$ is small. To quantify this, we will introduce an approximation $\widehat{h}_{\varepsilon}^{*}$ of $h_{\varepsilon}^{*}$ which depends locally on $h$ and prove a lemma (Lemma 2.1 ) to the effect that $\widehat{h}_{\varepsilon}^{*}$ and $\mathrm{h}_{\varepsilon}^{*}$ are close when $\varepsilon$ are small. This will be useful at several places in this section, especially for the proof of locality (essentially, Axiom II) in Sect. 2.5.

For $\varepsilon>0$, let $\psi_{\varepsilon}: \mathbb{C} \rightarrow[0,1]$ be a deterministic, smooth, radially symmetric bump function which is identically equal to 1 on $B_{\varepsilon^{1 / 2} / 2}(0)$ and vanishes outside of 
$B_{\varepsilon^{1 / 2}}(0)$ [in fact, the power $1 / 2$ could be replaced by any $\left.p \in(0,1)\right]$. We can choose $\psi_{\varepsilon}$ in such a way that $\varepsilon \mapsto \psi_{\varepsilon}$ is a continuous mapping from $(0, \infty)$ to the space of continuous functions on $\mathbb{C}$, equipped with the uniform topology. Recalling that $p_{s}(z, w)$ denotes the heat kernel, we define

$$
\widehat{\mathrm{h}}_{\varepsilon}^{*}(z):=\int_{\mathbb{C}} \psi_{\varepsilon}(z-w) \mathrm{h}(w) p_{\varepsilon^{2} / 2}(z, w) d w,
$$

with the integral interpreted in the sense of distributional pairing. Since $\psi_{\varepsilon}$ vanishes outside of $B_{\varepsilon^{1 / 2}}(0)$, we have that $\widehat{h}_{\varepsilon}^{*}(z)$ is a.s. determined by $\left.\mathrm{h}\right|_{B_{\varepsilon^{1 / 2}}(z)}$. It is easy to see that $\widehat{h}_{\varepsilon}^{*}$ a.s. admits a continuous modification (see Lemma 2.1). We henceforth assume that $\widehat{h}_{\varepsilon}^{*}$ is replaced by such a modification.

As in (1.5), we define the localized LFPP metric

$$
\widehat{D}_{\mathrm{h}}^{\varepsilon}(z, w):=\inf _{P: z \rightarrow w} \int_{0}^{1} e^{\xi \widehat{\mathrm{h}}_{\varepsilon}^{*}(P(t))}\left|P^{\prime}(t)\right| d t
$$

where the infimum is over all piecewise continuously differentiable paths from $z$ to $w$. By the definition of $\widehat{h}_{\varepsilon}^{*}$,

for any open $U \subset \mathbb{C}$, the internal metric $\widehat{D}_{\mathrm{h}}^{\varepsilon}(\cdot, \cdot ; U)$ is a.s. determined by $\left.\mathrm{h}\right|_{B_{\varepsilon^{1 / 2}}(U)}$.

Lemma 2.1 Let $h$ be a GFF plus a bounded continuous function. Then a.s. $(z, \varepsilon) \mapsto$ $\widehat{h}_{\varepsilon}^{*}(z)$ is continuous. Furthermore, for each bounded open set $U \subset \mathbb{C}$, a.s.

$$
\lim _{\varepsilon \rightarrow 0} \sup _{z \in \bar{U}}\left|h_{\varepsilon}^{*}(z)-\widehat{h}_{\varepsilon}^{*}(z)\right|=0
$$

In particular, a.s.

$$
\lim _{\varepsilon \rightarrow 0} \frac{\widehat{D}_{h}^{\varepsilon}(z, w ; U)}{D_{h}(z, w ; U)}=1, \quad \text { uniformly over all } z, w \in U \text { with } z \neq w
$$

To prove Lemma 2.1, we will need the following elementary estimate for the circle average process, whose proof we postpone until after the proof of Lemma 2.1.

Lemma 2.2 Let $h$ be a whole-plane GFF (with any choice of additive constant) and let $\left\{h_{r}\right\}_{r \geq 0}$ be its circle average process. For each $R>0$ and $\zeta>0$, a.s.

$$
\sup _{z \in B_{R}(0)} \sup _{r>0} \frac{\left|h_{r}(z)\right|}{\max \left\{(2+\zeta) \log (1 / r),(\log r)^{1 / 2+\zeta}, 1\right\}}<\infty .
$$


Proof of Lemma 2.1 We first consider the case when $\mathrm{h}=h$ is a whole-plane GFF normalized so that $h_{1}(0)=0$. The functions $w \mapsto \psi_{\varepsilon}(z-w)$ and $w \mapsto p_{\varepsilon^{2} / 2}(z, w)$ are each radially symmetric about $z$, i.e., they depend only on $|z-w|$. Using the circle average process $\left\{h_{r}\right\}_{r>0}$, we may therefore write in polar coordinates

$$
h_{\varepsilon}^{*}(z)=\frac{2}{\varepsilon^{2}} \int_{0}^{\infty} r h_{r}(z) e^{-r^{2} / \varepsilon^{2}} d r \text { and } \widehat{h}_{\varepsilon}^{*}(z)=\frac{2}{\varepsilon^{2}} \int_{0}^{\varepsilon^{1 / 2}} r h_{r}(z) \psi_{\varepsilon}(r) e^{-r^{2} / \varepsilon^{2}} d r
$$

From this representation and the continuity of the circle average process, we infer that $(z, \varepsilon) \mapsto \widehat{h}_{\varepsilon}^{*}(z)$ a.s. admits a continuous modification.

Since $\psi_{\varepsilon} \equiv 1$ on $B_{\varepsilon^{1 / 2} / 2}(z)$ and $\psi_{\varepsilon}$ takes values in $[0,1]$,

$$
\left|h_{\varepsilon}^{*}(z)-\widehat{h}_{\varepsilon}^{*}(z)\right| \leq \frac{2}{\varepsilon^{2}} \int_{\varepsilon^{1 / 2} / 2}^{\infty} r\left|h_{r}(z)\right| e^{-r^{2} / \varepsilon^{2}} d r .
$$

By Lemma 2.2 (applied with $\zeta=1 / 2$, say), there is a random constant $C=C(U)>0$ such that $\left|h_{r}(z)\right| \leq C \max \{\log (1 / r), \log r, 1\}$ for each $z \in U$ and $r>0$. Plugging this into (2.8) shows that a.s.

$$
\sup _{z \in U}\left|h_{\varepsilon}^{*}(z)-\widehat{h}_{\varepsilon}^{*}(z)\right| \leq \frac{2 C}{\varepsilon^{2}} \int_{\varepsilon^{1 / 2}}^{\infty} r \max \{\log (1 / r), \log r, 1\} e^{-r^{2} / \varepsilon^{2}} d r
$$

which tends to zero exponentially fast as $\varepsilon \rightarrow 0$. This gives (2.4) in the case of a whole-plane GFF with $h_{1}(0)=0$.

If $f: \mathbb{C} \rightarrow \mathbb{R}$ is a bounded continuous function, we similarly obtain a.s. $\lim _{\varepsilon \rightarrow 0} \sup _{z \in U}\left|f_{\varepsilon}^{*}(z)-\widehat{f}_{\varepsilon}^{*}(z)\right|=0$, using the notation (1.1) and (2.1) with $f$ in place of $h$ or $\mathrm{h}$. This gives (2.4) in the case of a whole-plane GFF plus a bounded continuous function. The relation (2.5) is immediate from (2.2) and the definition of LFPP.

To conclude the proof of Lemma 2.1 we still need to prove Lemma 2.2. To deal with large values of $r$, we will use the following lemma.

Lemma 2.3 Let h be a whole-plane GFF. For each $R>0$ and $\zeta>0$, a.s.

$$
\lim _{r \rightarrow \infty} \sup _{z \in B_{R}(0)} \frac{\left|h_{r}(z)\right|}{(\log r)^{1 / 2+\zeta}}=0
$$

Proof The process $\left\{h_{r}(z)-h_{r}(0): z \in B_{R}(0), r \in[1 / 2,1]\right\}$ is centered Gaussian with variances bounded above by a constant depending only on $R$. Furthermore, this process a.s. admits a continuous modification [16, Proposition 3.1], so if we replace it by such a modification then a.s. $\sup _{z \in B_{R}(0)} \sup _{r \in[1 / 2,1]}\left|h_{r}(z)-h_{r}(0)\right|<$ $\infty$. By the Borel-TIS inequality [8,45] (see, e.g., [4, Theorem 2.1.1]), we have 
$\mathbb{E}\left[\sup _{z \in B_{R}(0)} \sup _{r \in[1 / 2,1]}\left|h_{r}(z)-h_{r}(0)\right|\right]<\infty$ and there are constants $c_{0}, c_{1}>0$ depending only on $R$ such that for each $A>0$,

$$
\mathbb{P}\left[\sup _{z \in B_{R}(0)} \sup _{r \in[1 / 2,1]}\left|h_{r}(z)-h_{r}(0)\right|>A\right] \leq c_{0} e^{-c_{1} A^{2}} .
$$

Note that we absorbed the $R$-dependent constant $\mathbb{E}\left[\sup _{z \in B_{R}(0)} \sup _{r \in[1 / 2,1]} \mid h_{r}(z)\right.$ $\left.-h_{r}(0) \mid\right]$ into $c_{0}$.

By the scale invariance of the law of $h$, viewed modulo an additive constant, we infer from (2.11) that for each $k \in \mathbb{N}_{0}$ and $A>0$,

$$
\mathbb{P}\left[\sup _{z \in B_{R 2^{k}}(0)} \sup _{r \in\left[2^{k-1}, 2^{k}\right]}\left|h_{r}(z)-h_{r}(0)\right|>A\right] \leq c_{0} e^{-c_{1} A^{2}}
$$

By applying this with $A$ equal to a universal constant times $k^{1 / 2+\zeta / 2}$, say, then using the Borel-Cantelli lemma, we get that a.s.

$$
\lim _{k \rightarrow \infty} \sup _{z \in B_{R 2^{k}}(0)} \sup _{r \in\left[2^{k-1}, 2^{k}\right]} \frac{\left|h_{r}(z)-h_{r}(0)\right|}{(\log r)^{1 / 2+\zeta}}=0 .
$$

Each $z \in K$ is contained in $B_{R 2^{k}}(0)$ for each $k \in \mathbb{N}$ and each $r \geq 1 / 2$ is contained in $\left[2^{k-1}, 2^{k}\right]$ for some $k \in \mathbb{N}$. Hence, (2.13) implies that a.s.

$$
\lim _{r \rightarrow \infty} \sup _{z \in B_{R}(0)} \frac{\left|h_{r}(z)-h_{r}(0)\right|}{(\log r)^{1 / 2+\zeta}}=0 .
$$

Since $t \mapsto h_{e^{t}}(0)$ is a standard two-sided linear Brownian motion [16, Section 3], it follows that a.s. $\left|h_{r}(0)\right| /(\log r)^{1 / 2+\zeta} \rightarrow 0$ as $r \rightarrow \infty$. Combining this with (2.14) yields (2.10).

Proof of Lemma 2.2 Standard estimates for the maximum of the circle average process (see, e.g., the proof of [32, Lemma 3.1]) show that a.s.

$$
\sup _{z \in B_{R}(0)} \sup _{r \in(0,1 / 2]} \frac{\left|h_{r}(z)\right|}{(2+\zeta) \log (1 / r)}<\infty .
$$

By the continuity of the circle average process, a.s. for any $r_{0}>1 / 2, \sup _{z \in B_{R}(0)}$ $\sup _{r \in\left[1 / 2, r_{0}\right]}\left|h_{r}(z)\right|<\infty$. By Lemma 2.3, it is a.s. the case that for each large enough $r_{0}>0$,

$$
\sup _{z \in B_{R}(0)} \sup _{r \geq r_{0}} \frac{\left|h_{r}(z)\right|}{(\log r)^{1 / 2+\zeta}}<\infty
$$

Combining these estimates gives (2.6). 


\subsection{Subsequential limits}

In this subsection we explain why the results of [10] imply that the laws of the re-scaled LFPP metrics $\mathfrak{a}_{\varepsilon}^{-1} D_{\mathrm{h}}^{\varepsilon}$ are tight (this is not entirely immediate since [10] considers a slightly different class of fields and only looks at metrics on bounded domains). We will in fact obtain a stronger convergence statement which also includes the convergence of internal metrics of $\mathfrak{a}_{\varepsilon}^{-1} D_{\mathrm{h}}^{\varepsilon}$ on a certain class of sub-domains of $\mathbb{C}$.

Definition 2.4 (Dyadic domain) A closed square $S \subset \mathbb{C}$ is dyadic if $S$ has side length $2^{k}$ and corners in $2^{k} \mathbb{Z}^{2}$ for some $k \in \mathbb{Z}$. We say that $W \subset \mathbb{C}$ is a dyadic domain if there exists a finite collection of dyadic squares $\mathcal{S}$ such that $W$ is the interior of $\bigcup_{S \in \mathcal{S}} S$. Note that a dyadic domain is a bounded open set.

Lemma 2.5 Let $\mathrm{h}$ be a whole-plane GFF plus a bounded continuous function.

A. The laws of the metrics $\mathfrak{a}_{\varepsilon}^{-1} D_{h}^{\varepsilon}$ are tight w.r.t. the local uniform topology on $\mathbb{C} \times \mathbb{C}$ and any subsequential limit of these laws is supported on continuous length metrics on $\mathbb{C}$.

B. Let $\mathcal{W}$ be the (countable) set of all dyadic domains. For any sequence of positive $\varepsilon$ 's tending to zero, there is a subsequence $\mathcal{E}$ and a coupling of a continuous length metric $D_{h}$ on $\mathbb{C}$ and a length metric $D_{h, W}$ on $\bar{W}$ for each $W \in \mathcal{W}$ which induces the Euclidean topology on $\bar{W}$ such that the following is true. Along $\mathcal{E}$, we have the convergence of joint laws

$$
\left(\mathfrak{a}_{\varepsilon}^{-1} D_{h}^{\varepsilon},\left\{\mathfrak{a}_{\varepsilon}^{-1} D_{h}^{\varepsilon}(\cdot, \cdot ; \bar{W})\right\}_{W \in \mathcal{W}}\right) \rightarrow\left(D_{h},\left\{D_{h, W}\right\}_{W \in \mathcal{W}}\right)
$$

where the first coordinate is given the local uniform topology on $\mathbb{C} \times \mathbb{C}$ and each element of the collection in the second coordinate is given the uniform topology on $\bar{W} \times \bar{W}$. Furthermore, for each $W \in \mathcal{W}$ we have the a.s. equality of internal metrics $D_{h, W}(\cdot, \cdot ; W)=D_{h}(\cdot, \cdot ; W)$.

In the setting of Assertion $\mathrm{A}$, we note that the space of continuous functions $\mathbb{C} \times \mathbb{C} \rightarrow \mathbb{R}$, equipped with the local uniform topology, is separable and completely metrizable, which means that we can apply Prokhorov's theorem in this space. Assertion B of Lemma 2.5 does not give that $D_{\mathrm{h}}^{\varepsilon}(\cdot, \cdot ; \bar{W}) \rightarrow D_{\mathrm{h}}(\cdot, \cdot ; \bar{W})$ in law along $\mathcal{E}$ for each $W \in \mathcal{W}$. The reason why we do not prove this statement is to avoid worrying about possible pathologies near $\partial W$ (see Lemma 2.11). We now proceed with the proof of Lemma 2.5. At several places in this section, we will use the following elementary scaling relation for LFPP.

Lemma 2.6 Let $h$ be a whole-plane GFF normalized so that $h_{1}(0)=0$. Let $r>0$ and let $h^{r}:=h(r \cdot)-h_{r}(0)$, so that $h^{r} \stackrel{d}{=} h$. The LFPP metrics defined as in (1.5) for $h$ and $h^{r}$ are related by

$D_{h^{r}}^{\varepsilon / r} \stackrel{d}{=} D_{h}^{\varepsilon / r} \quad$ and $\quad D_{h^{r}}^{\varepsilon / r}(z, w)=r^{-1} e^{-\xi h_{r}(0)} D_{h}^{\varepsilon}(r z, r w), \quad \forall \varepsilon>0, \quad \forall z, w \in \mathbb{C}$ 
Proof Using the notation (1.1), we get from a standard change of variables that the convolutions of $h^{r}$ and $h$ with the heat kernel satisfy $h_{\varepsilon / r}^{r, *}(z)=h_{\varepsilon}^{*}(r z)-h_{r}(0)$ for each $\varepsilon>0$ and $z \in \mathbb{C}$. Using the definition (1.5) of LFPP, we now compute

$$
\begin{aligned}
e^{-\xi h_{r}(0)} D_{h}^{\varepsilon}(r z, r w) & =\inf _{P: r z \rightarrow r w} \int_{0}^{1} e^{\xi\left(h_{\varepsilon}^{*}(P(t))-h_{r}(0)\right)}\left|P^{\prime}(t)\right| d t \\
& =\inf _{P: r z \rightarrow r w} \int_{0}^{1} e^{\xi h_{\varepsilon / r}^{r, *}(P(t) / r)}\left|P^{\prime}(t)\right| d t \\
& =r \inf _{\widetilde{P}: z \rightarrow w} \int_{0}^{1} e^{\xi h_{\varepsilon / r}^{r, *}(\widetilde{P}(t))}\left|\widetilde{P}^{\prime}(t)\right| d t \quad(\text { set } \widetilde{P}=P / r) \\
& =r D_{h^{r}}^{\varepsilon / r}(z, w) .
\end{aligned}
$$

To check that our limiting metrics are length metrics, we will need the following standard fact from metric geometry.

Lemma 2.7 Let $X$ be a compact topological space and let $\left\{D^{n}\right\}_{n \in \mathbb{N}}$ be a sequence of length metrics on $X$ which converge uniformly to a metric $D$ on $X$. Then $D$ is a length metric on $X$.

Proof This is [5, Exercise 2.4.19], which in turn is an easy consequence of [5, Corollary 2.4.17].

Let us now record what we get from [10].

Lemma 2.8 Let $S \subset \mathbb{C}$ be a closed square and let $h$ be a whole-plane GFF plus a bounded continuous function. The laws of the internal metrics $\mathfrak{a}_{\varepsilon}^{-1} D_{h}^{\varepsilon}(\cdot, \cdot ; S)$ for $\varepsilon \in(0,1)$ are tight w.r.t. the uniform topology on $S \times S$ and any subsequential limit of these laws is supported on length metrics which induce the Euclidean topology on $S$.

Proof We first consider the case when $S=[0,1]^{2}$ is the Euclidean unit square and $\mathrm{h}=h$ is a whole-plane GFF normalized so that $h_{1}(0)=0$. Let $\stackrel{\circ}{h}$ be a zero-boundary GFF on $(-1,2)^{2}$. By the Markov property of the whole-plane GFF, we can couple $h$ and $h$ in such a way that $h-\grave{h}$ is a.s. harmonic, hence continuous, on $(-1,2)^{2}$.

Recall the heat kernel $p_{s}(z, w)=\frac{1}{2 \pi s} e^{-|z-w| /(2 s)}$. For $z \in[0,1]^{2}$ and $\varepsilon \in(0,1)$, we define the convolution $\stackrel{\circ}{h}_{\varepsilon}^{*}=\stackrel{\circ}{h} * p_{\varepsilon^{2} / 2}$ as in (1.1). For $z, w \in(-1,2)^{2}$, define $D_{h}^{\varepsilon}(z, w)$ as in (1.5) with $h_{\varepsilon}^{*}$ in place of $h_{\varepsilon}^{*}$. It is shown in [10, Theorem 1] (see also [10, Section 6.1]) that there are constants $\left\{\lambda_{\varepsilon}\right\}_{\varepsilon>0}$ such that the internal metrics $\lambda_{\varepsilon}^{-1} D_{h}^{\varepsilon}\left(\cdot, \cdot ;[0,1]^{2}\right)$ are tight w.r.t. the uniform topology on $[0,1]^{2} \times[0,1]^{2}$ and any subsequential limit of these laws is supported on length metrics which induce the Euclidean topology on $[0,1]^{2}$.

We now want to compare $D_{h}^{\varepsilon}$ and $D_{h}^{\varepsilon}$ using the fact that $\left.(h-h)\right|_{(-1,2)^{2}}$ is a continuous function. However, we cannot do this directly since we only have a uniform bound for $h-\stackrel{\circ}{h}$ on compact subsets of $(-1,2)^{2}$ and the convolution (1.1) does not depend 
locally on the field. To this end, we define the localized LFPP metrics $\widehat{D}_{h}^{\varepsilon}$ and $\widehat{D}_{h}^{\varepsilon}$ as in (2.2) with $\mathrm{h}=h$ and with $h$ in place of $h$, respectively. Then Lemma 2.1 remains true with $D_{h}^{\varepsilon}$ and $\widehat{D}_{\stackrel{h}{\varepsilon}}^{\varepsilon}$ in place of $D_{h}^{\varepsilon}$ and $\widehat{D}_{h}^{\varepsilon}$ and with $U$ any open set satisfying $\bar{U} \subset(-1,2)^{2}$, with the same proof (actually, the proof is simpler since one does not need Lemma 2.3). Therefore, a.s. $\widehat{D}_{h}^{\varepsilon}(z, w ; U) / D_{h}^{\varepsilon}(z, w ; U) \rightarrow 1$ uniformly over all distinct $z, w \in U$ and the conclusion of the preceding paragraph is true with $\widehat{D}_{h}^{\varepsilon}$ in place of $D_{h}^{\varepsilon}$.

Since $h-\stackrel{\circ}{h}$ is a.s. equal to a continuous function on a neighborhood of $[0,1]^{2}$, we infer from $(2.3)$ that a.s. the metrics $\widehat{D}_{h}^{\varepsilon}\left(\cdot, \cdot ;[0,1]^{2}\right)$ and $\widehat{D}_{h}^{\varepsilon}\left(\cdot, \cdot ;[0,1]^{2}\right)$ are biLipschitz equivalent with (random) $\varepsilon$-independent Lipschitz constants. By combining this with the conclusion of the preceding paragraph and Lemma 2.7, we get that the laws of the internal metrics $\lambda_{\varepsilon}^{-1} D_{h}^{\varepsilon}(\cdot, \cdot ; S)$ for $\varepsilon \in(0,1)$ are tight w.r.t. the uniform topology on $[0,1]^{2} \times[0,1]^{2}$ and any subsequential limit of these laws is supported on length metrics which induce the Euclidean topology on $S$. In particular, this implies that $\lambda_{\varepsilon}$ is bounded above and below by $\varepsilon$-independent constants times the median $\widehat{D}_{h}^{\varepsilon}$-distance between the left and right sides of $[0,1]^{2}$. By Lemma 2.1 (for $h$ ), we now get that $\left\{\mathfrak{a}_{\varepsilon} / \lambda_{\varepsilon}\right\}_{\varepsilon \in(0,1)}$ is bounded above and below by positive, finite constants and the statement of the lemma holds in the special case when $\mathrm{h}=h$ and $S=[0,1]^{2}$.

By Lemma 2.6 and the scale and translation invariance of the law of $h$, modulo additive constant, this implies the statement of the lemma for a general choice of $S$, but still with $\mathrm{h}=h$. If $h$ is a whole-plane GFF and $f$ is a bounded continuous function, then the metrics $D_{h+f}^{\varepsilon}$ and $D_{h}^{\varepsilon}$ are bi-Lipschitz equivalent, with Lipschitz constants $e^{ \pm \xi\|f\|_{\infty}}$. Hence the case of a whole-plane GFF implies the case of a whole-plane GFF plus a continuous function.

We now upgrade from internal metrics on closed squares to internal metrics on closures of dyadic domains.

Lemma 2.9 Let $W \subset \mathbb{C}$ be a dyadic domain. The laws of the internal metrics $\mathfrak{a}_{\varepsilon}^{-1} D_{h}^{\varepsilon}(\cdot, \cdot ; \bar{W})$ for $\varepsilon \in(0,1)$ are tight w.r.t. the uniform topology on $\bar{W} \times \bar{W}$ and any subsequential limit of these laws is supported on length metrics which induce the Euclidean topology on $\bar{W}$.

Proof If $W$ is a dyadic domain, then $\bar{W}$ has finitely many connected components and these connected components are the closures of dyadic domains which lie at positive Euclidean distance from each other. By considering each connected component separately, we can assume without loss of generality that $\bar{W}$ is connected.

For a connected set $X \subset \mathbb{C}$, a collection $\mathcal{D}$ of random metrics on $X$ is tight w.r.t. the local uniform topology if and only if for each $\zeta>0$, there exists $\delta>0$ such that for each $d \in \mathcal{D}$, it holds with probability at least $1-\zeta$ that

$$
d(z, w) \leq \zeta, \quad \forall z, w \in X \quad \text { such that }|z-w| \leq \delta
$$

Indeed, this is an easy consequence of the Arzéla-Ascoli theorem, the Prokhorov theorem, and the triangle inequality. 
For any closed square $S \subset \bar{W}$, the restriction of $D_{\mathrm{h}}^{\varepsilon}(\cdot, \cdot ; \bar{W})$ to $S$ is bounded above by the internal metric of $D_{\mathrm{h}}^{\varepsilon}(\cdot, \cdot ; \bar{W})$ on $S$, which equals $D_{\mathrm{h}}^{\varepsilon}(\cdot, \cdot ; S)$. By Lemma 2.8 and the above tightness criterion, the laws of the restrictions of $\left\{\mathfrak{a}_{\varepsilon}^{-1} D_{\mathrm{h}}^{\varepsilon}(\cdot, \cdot ; \bar{W})\right\}_{\varepsilon \in(0,1)}$ to $S$ are tight. Since $W$ is a dyadic domain, we can choose a finite collection $\mathcal{S}$ of closed squares such that $\bigcup_{S \in \mathcal{S}} S=\bar{W}$.

By the above tightness criterion applied to each square in $\mathcal{S}$, for each $\zeta>0$, there exists $\delta>0$ such that for each $\varepsilon \in(0,1)$, it holds with probability at least $1-\zeta$ that

$$
\mathfrak{a}_{\varepsilon}^{-1} D_{\mathrm{h}}^{\varepsilon}(z, w ; \bar{W}) \leq \zeta, \quad \forall z, w \in \bar{W} \quad \text { s.t. } \quad|z-w| \leq \delta \quad \text { and } \quad z, w \in S \text { for some } S \in \mathcal{S} .
$$

Now assume that (2.20) holds and consider points $z, w \in \bar{W}$ such that $|z-w| \leq \delta / 2$ but $z$ and $w$ do not lie in the same square of $\mathcal{S}$. If $\delta$ is sufficiently small (depending only on the collection of squares $\mathcal{S}$ ), then we can find squares $S, S^{\prime} \in \mathcal{S}$ such that $z \in S, w \in S^{\prime}$, and $S \cap S^{\prime} \neq \emptyset$. Since $S$ and $S^{\prime}$ are closed squares, geometric considerations show that there is a $u \in S \cap S^{\prime}$ such that $|z-u| \leq \delta$ and $|w-u| \leq \delta$. By (2.20) and the triangle inequality this implies that $\mathfrak{a}_{\varepsilon}^{-1} D_{\mathrm{h}}^{\varepsilon}(z, w ; \bar{W}) \leq 2 \zeta$. Therefore, $\forall \varepsilon \in(0,1)$ it holds with probability at least $1-\zeta$ that

$$
\mathfrak{a}_{\varepsilon}^{-1} D_{\mathrm{h}}^{\varepsilon}(z, w ; \bar{W}) \leq 2 \zeta, \quad \forall z, w \in \bar{W} \text { such that }|z-w| \leq \delta / 2
$$

Since $\zeta$ is arbitrary, the above tightness criterion applied on all of $\bar{W}$ now shows that the laws of the metrics $\mathfrak{a}_{\varepsilon}^{-1} D_{\mathrm{h}}^{\varepsilon}(\cdot, \cdot ; W)$ for $\varepsilon \in(0,1)$ are tight w.r.t. the uniform topology on $\bar{W} \times \bar{W}$.

Let $\widetilde{D}$ be a subsequential limit of $\mathfrak{a}_{\varepsilon}^{-1} D_{\mathrm{h}}^{\varepsilon}(\cdot, \cdot ; W)$ in law w.r.t. the local uniform topology. A priori $\widetilde{D}$ might be a pseudometric, not a metric. We need to show that $\widetilde{D}$ is in fact a length metric and that it induces the Euclidean topology on $\bar{W}$. To this end, consider two squares (not necessarily dyadic) $S_{1} \subset S_{2} \subset \bar{W}$ such that $S_{1}$ lies at positive Euclidean distance from $\partial S_{2} \backslash \partial W$. For each $\varepsilon>0$, we have $D_{\mathrm{h}}^{\varepsilon}\left(S_{1}, W \backslash S_{2} ; \bar{W}\right)=$ $D_{\mathrm{h}}^{\varepsilon}\left(S_{1}, \partial S_{2} \backslash \partial W ; S_{2}\right)$ and $D_{\mathrm{h}}^{\varepsilon}\left(S_{1}, W \backslash S_{2} ; \bar{W}\right) \rightarrow \widetilde{D}\left(S_{1}, W \backslash S_{2}\right)$ in law. From this and Lemma 2.8, we infer that a.s. $\widetilde{D}\left(S_{1}, W \backslash S_{2}\right)>0$. By considering an appropriate countable collection of such square annuli whose inner squares $S_{1}$ cover $\bar{W}$, we infer that a.s. $\widetilde{D}(u, v)>0$ whenever $u, v \in \bar{W}$ with $u \neq v$. This implies that $\widetilde{D}$ is a metric. Since $\bar{W}$ is compact, it follows that $\widetilde{D}$ induces the Euclidean topology on $\bar{W}$. By Lemma 2.7, $\widetilde{D}$ is a length metric.

The following lemma will allow us to extract tightness of $\mathfrak{a}_{\varepsilon}^{-1} D_{\mathrm{h}}^{\varepsilon}$ from tightness of $\mathfrak{a}_{\varepsilon}^{-1} D_{\mathrm{h}}^{\varepsilon}(\cdot, \cdot ; S)$ for squares $S \subset \mathbb{C}$.

Lemma 2.10 For $r>0$, let $S_{r}(0)$ be the closed square of side length $r$ centered at zero. Let $h$ be a whole-plane GFF plus a bounded continuous function. For each $p \in(0,1)$ and each $C>0$, there exists $R=R(p, C)>1$ (depending on $p, C$ and the law of $h$ ) such that for each fixed $r>0$,

$$
\liminf _{\varepsilon \rightarrow 0} \mathbb{P}\left[\sup _{u, v \in S_{r}(0)} D_{h}^{\varepsilon}(u, v)<\frac{1}{C} D_{h}^{\varepsilon}\left(S_{r}(0), \partial S_{R r}(0)\right)\right] \geq p .
$$


Proof We first consider the case when $\mathrm{h}=h$ is a whole-plane GFF normalized so that $h_{1}(0)=0$. By Lemma 2.8 applied with $\bar{W}=S_{1}(0)$, there exists $R=R(p, C)>1$ such

$$
\liminf _{\varepsilon \rightarrow 0} \mathbb{P}\left[\sup _{u, v \in S_{1 / R}(0)} D_{h}^{\varepsilon}(u, v)<\frac{1}{C} D_{h}^{\varepsilon}\left(S_{1 / R}(0), \partial S_{1}(0)\right)\right] \geq p .
$$

The occurrence of the event in (2.22) is unaffected by re-scaling $D_{h}^{\varepsilon}$ by a constant factor. By Lemma 2.6 applied with $R r$ in place of $r$, we see that (2.22) implies that for each fixed $r>0$,

$$
\liminf _{\varepsilon \rightarrow 0} \mathbb{P}\left[\sup _{u, v \in S_{r}(0)} D_{h}^{\varepsilon}(u, v)<\frac{1}{C} D_{h}^{\varepsilon}\left(S_{r}(0), \partial S_{R r}(0)\right)\right] \geq p .
$$

Now suppose that $\mathrm{h}=h+f$ is a whole-plane GFF plus a bounded continuous function. If $f$ is a (possibly random) bounded continuous function, then $D_{h+f}^{\varepsilon}$ and $D_{h}^{\varepsilon}$ are a.s. bi-Lipschitz equivalent with Lipschitz constants $e^{-\xi\|f\|_{\infty}}$ and $e^{\xi\|\|_{\infty}}$. Furthermore, since $f$ is a.s. bounded exists a deterministic $A>1$ such that $\mathbb{P}\left[e^{\xi\|f\|_{\infty}} \leq A\right] \geq p$. By (2.23) with $A^{2} C$ in place of $C$, we get (2.21) but with $1-2(1-p)$ in place of $p$. Since $p$ can be made arbitrarily close to 1 , this yields (2.21).

The last lemma we need for the proof of Lemma 2.5 is the following deterministic compatibility statement for limits of internal metrics, which is used to get the relationship between internal metrics in assertion B of Lemma 2.5.

Lemma 2.11 Let $V \subset U \subset \mathbb{C}$ be open. Let $\left\{D^{n}\right\}_{n \in \mathbb{N}}$ be a sequence of continuous length metrics on $U$ which converges to a continuous length metric $D$ (w.r.t. the local uniform topology on $U \times U)$. Suppose also that $D^{n}(\cdot, \cdot ; \bar{V})$ converges to a continuous length metric $\widetilde{D}$ w.r.t. the uniform topology on $\bar{V} \times \bar{V}$. Then $D(\cdot, \cdot ; V)=\widetilde{D}(\cdot, \cdot ; V)$.

In the setting of Lemma 2.11 , we do not necessarily have $D(\cdot, \cdot ; \bar{V})=\widetilde{D}$. The reason is that it could be, e.g., that paths of near-minimal $\widetilde{D}$-length spend a positive fraction of their time in $\partial V$.

Proof of Lemma 2.11 Let $u, v \in V$ such that $D(u, v)<D(u, \partial V)$. Since $D$ is a length metric, $D(u, v)=D(u, v ; V)=D(u, v ; \bar{V})$. Furthermore, for large enough $n \in \mathbb{N}$ we have $D^{n}(u, v)<D^{n}(u, \partial V)$ which implies that $D^{n}(u, v)=D^{n}(u, v ; V)=$ $D^{n}(u, v ; \bar{V})$. Therefore, $D^{n}(u, v)$ converges to both $D(u, v)=D(u, v ; V)$ and $\widetilde{D}(u, v)$. Furthermore, we have $\widetilde{D}(u, v)<\widetilde{D}(u, v ; \partial V)$ which implies that $\widetilde{D}(u, v)=$ $\widetilde{D}(u, v ; V)$. Consequently, $D(u, v ; V)=\widetilde{D}(u, v ; V)$ for each $u, v \in V$ with $D(u, v)<D(u, \partial V)$. This implies that the $D$-length of any path in $V$ which lies at positive Euclidean distance from $\partial V$ is the same as its $\widetilde{D}$-length. Since $D(\cdot, \cdot ; V)$ and $\widetilde{D}(\cdot, \cdot ; V)$ are length metrics, we conclude that $D(\cdot, \cdot ; V)=\widetilde{D}(\cdot, \cdot ; V)$.

Proof of Lemma 2.5 For $r>0$, let $S_{r}(0)$ be the closed square of side length $r$ centered at zero, as in Lemma 2.10. Let $p \in(0,1)$ and let $R=R(p)>1$ be as in Lemma 2.10 
with $C=2$ and with $(1+p) / 2$, say, in place of $p$. Then for each fixed $r>0$ and each small enough $\varepsilon>0$, it holds with probability at least $p$ that

$$
\begin{aligned}
& \sup _{u, v \in S_{r}(0)} D_{\mathrm{h}}^{\varepsilon}(u, v) \leq \frac{1}{2} D_{\mathrm{h}}^{\varepsilon}\left(S_{r}(0), \partial S_{R r}(0)\right) \\
& \text { which implies } D_{\mathrm{h}}^{\varepsilon}(u, v)=D_{\mathrm{h}}^{\varepsilon}\left(u, v ; S_{R r}(0)\right), \quad \forall u, v \in S_{r}(0) .
\end{aligned}
$$

We now apply Lemma 2.8 with $S=S_{R r}(0)$ and use that $p$ can be made arbitrarily close to 1 to get that the laws of $\left.\mathfrak{a}_{\varepsilon}^{-1} D_{\mathrm{h}}^{\varepsilon}\right|_{S_{r}(0)}$ are tight w.r.t. the local uniform topology on $S_{r}(0)$. Furthermore, any subsequential limit in law of these metrics a.s. induces the Euclidean topology on $S_{r}(0)$. Since $r$ can be made arbitrarily large, we get that the metrics $\mathfrak{a}_{\varepsilon}^{-1} D_{\mathrm{h}}^{\varepsilon}$ are tight w.r.t. the local uniform topology on $\mathbb{C} \times \mathbb{C}$ and any subsequential limit in law is a.s. a continuous metric on $\mathbb{C}$.

To prove assertion A, it remains to check that if $D_{\mathrm{h}}$ is a subsequential limit in law of the metrics $\mathfrak{a}_{\varepsilon}^{-1} D_{\mathrm{h}}^{\varepsilon}$, then a.s. $D_{\mathrm{h}}$ is a length metric. To this end, let $p \in(0,1)$ and let $R=R(p)>1$ be as above. By Lemma 2.8, if we are given $r>0$ then by possibly passing to a further subsequence we can arrange that along our subsequence, the joint law of $\left(\mathfrak{a}_{\varepsilon}^{-1} D_{\mathrm{h}}^{\varepsilon}, \mathfrak{a}_{\varepsilon}^{-1} D_{\mathrm{h}}^{\varepsilon}\left(\cdot, \cdot ; S_{R r}(0)\right)\right)$ converges to a coupling $\left(D_{\mathrm{h}}, \widetilde{D}\right)$ where $\widetilde{D}$ is a length metric on $S_{R r}(0)$. By passing to the (subsequential) limit in (2.24), we get that with probability at least $p$,

$$
\sup _{u, v \in S_{r}(0)} D_{\mathrm{h}}(u, v) \leq \frac{1}{2} D_{\mathrm{h}}\left(S_{r}(0), \partial S_{R r}(0)\right) \quad \text { and } \quad D_{\mathrm{h}}(u, v)=\widetilde{D}(u, v), \quad \forall u, v \in S_{r}(0) .
$$

By Lemma 2.11, a.s. the internal metrics of $D_{\mathrm{h}}$ and $\widetilde{D}$ on the interior of $S_{R r}(0)$ coincide. Hence (2.24) implies that with probability at least $p, D_{\mathrm{h}}(u, v)$ is equal to the infimum of the $D_{\mathrm{h}}$-lengths of all continuous paths from $u$ to $v$ which are contained in the interior of $S_{R r}(0)$, which (by the first condition in (2.24)) is equal to the infimum of the $D_{\mathrm{h}}$-lengths of all continuous paths from $u$ to $v$. Since $p$ can be made arbitrarily close to 1 and $r$ can be made arbitrarily large, we get that a.s. $D_{\mathrm{h}}$ is a length metric.

To get the joint convergence (2.17), we first apply Lemma 2.9 and the Prokhorov theorem to get that the joint law of the metrics on the left side of (2.17) is tight. Moreover any subsequential limit of these joint laws is a coupling of a continuous length metric $D_{\mathrm{h}}$ on $\mathbb{C}$ and a length metric $D_{\mathrm{h}, W}$ on $\bar{W}$ for each $W \in \mathcal{W}$ which induces the Euclidean topology on $\bar{W}$. We then apply Lemma 2.11 to say that $D_{\mathrm{h}, W}(\cdot, \cdot ; W)=$ $D_{\mathrm{h}}(\cdot, \cdot ; W)$ for each $W \in \mathcal{W}$.

\subsection{Weyl scaling}

The following lemma will be used to check Axiom III.

Lemma 2.12 Let $h$ be a whole-plane GFF plus a bounded continuous function and consider a sequence $\varepsilon_{n} \rightarrow 0$ along which $\mathfrak{a}_{\varepsilon_{n}}^{-1} D_{h}^{\varepsilon_{n}}$ converges in law to some metric $D_{h}$ w.r.t. the local uniform topology. Suppose we have, using the Skorokhod theorem, coupled so this convergence occurs a.s. Then, a.s., for every sequence of bounded 
continuous functions $f^{n}: \mathbb{C} \rightarrow \mathbb{R}$ such that $f^{n}$ converges to a bounded continuous function $f$ uniformly on compact subsets of $\mathbb{C}$, we have the local uniform convergence $D_{h+f^{n}}^{\varepsilon_{n}} \rightarrow e^{\xi f} \cdot D_{h}$, where here $D_{h+f^{n}}^{\varepsilon}$ is defined as in (1.5) with $h+f^{n}$ in place of $h$ and $e^{\xi f} \cdot D_{h}$ is defined as in (1.7).

As a consequence of Lemma 2.12, if $\mathrm{h}$ is a whole-plane GFF plus a bounded continuous function and $\varepsilon_{n} \rightarrow 0$ is a sequence along which $\mathfrak{a}_{\varepsilon_{n}}^{-1} D_{\mathrm{h}}^{\varepsilon_{n}} \rightarrow D_{\mathrm{h}}$ in law, then whenever $\mathrm{h}^{\prime}$ is another whole-plane GFF plus a bounded continuous function, we have $\mathfrak{a}_{\varepsilon_{n}}^{-1} D_{\mathrm{h}^{\prime}}^{\varepsilon_{n}} \rightarrow D_{\mathrm{h}^{\prime}}$ in law for some limiting metric $D_{\mathrm{h}^{\prime}}$. Furthermore, $\left(\mathrm{h}, \mathrm{h}^{\prime}, D_{\mathrm{h}}, D_{\mathrm{h}^{\prime}}\right)$ can be coupled together in such a way that $\mathrm{h}^{\prime}-\mathrm{h}$ is a bounded continuous function and $D_{\mathrm{h}^{\prime}}=e^{\xi\left(\mathrm{h}^{\prime}-\mathrm{h}\right)} \cdot D_{\mathrm{h}}$. Consequently, any subsequence along which $\mathfrak{a}_{\varepsilon_{n}}^{-1} D_{\mathrm{h}}^{\varepsilon_{n}}$ converges in law gives us a way to define a metric associated with any whole-plane GFF plus a bounded continuous function.

Proof of Lemma 2.12 Let $f_{\varepsilon_{n}}^{*, n}=f^{n} * p_{\varepsilon_{n}^{2} / 2}$ be defined as in (1.1) with with $f^{n}$ in place of $h$. Then $f_{\varepsilon_{n}}^{*, n} \rightarrow f$ uniformly on compact subsets of $\mathbb{C}$. By the definition (1.5) of LFPP, we have $D_{\mathrm{h}+f^{n}}^{\varepsilon_{n}}=e^{\xi f_{\varepsilon_{n}}^{*, n}} \cdot D_{\mathrm{h}}^{\varepsilon_{n}}$.

We now want to apply an argument as in the proof of [11, Lemma 7.1] to say that $D_{\mathrm{h}+f^{n}}^{\varepsilon_{n}} \rightarrow e^{\xi f} \cdot D_{\mathrm{h}}$ w.r.t. the local uniform topology. That lemma only applies for metrics defined on squares, so we need to localize. We do this by means of Lemma 2.10. By taking a limit as $\varepsilon \rightarrow 0$ in the estimate of Lemma 2.10, then sending $p \rightarrow 1$, we find that a.s. for each $r>0$ and each $C>1$, there exists $r^{\prime}=r^{\prime}(r, C)>0$ (random) such that

$$
\sup _{u, v \in S_{r}(0)} D_{\mathrm{h}}(u, v) \leq \frac{1}{2 C} D_{\mathrm{h}}\left(S_{r}(0), \partial S_{r^{\prime}}(0)\right) .
$$

Furthermore, the uniform convergence $\mathfrak{a}_{\varepsilon_{n}}^{-1} D_{\mathrm{h}}^{\varepsilon_{n}} \rightarrow D_{\mathrm{h}}$, we get that (2.26) is a.s. true with $\mathfrak{a}_{\varepsilon_{n}}^{-1} D_{\mathrm{h}}^{\varepsilon_{n}}$ in place of $D_{\mathrm{h}}$ for large enough $n \in \mathbb{N}$, but with $C$ instead of $2 C$. This implies that each path of near-minimal $D_{\mathrm{h}}$-length between two points of $S_{r}(0)$ is contained in $S_{r^{\prime}}(0)$, and the same is true with $\mathfrak{a}_{\varepsilon_{n}}^{-1} D_{\mathrm{h}}^{\varepsilon_{n}}$ in place of $D_{\mathrm{h}}$ for large enough $n \in \mathbb{N}$. If we choose $C>\sup _{n \in \mathbb{N}}\left\|f^{n}\right\|_{\infty}$, then from (2.26) we deduce that each path of near-minimal $e^{\xi f} \cdot D_{\mathrm{h}}$-length between two points of $S_{r}(0)$ is contained in $S_{r^{\prime}}(0)$, and the same is true with $\mathfrak{a}_{\varepsilon_{n}}^{-1} D_{\mathrm{h}+f^{n}}^{\varepsilon_{n}}$ in place of $D_{\mathrm{h}}$ for large enough $n \in \mathbb{N}$. With these conditions in hand, the lemma now follows from the same proof as in [11, Lemma 7.1].

\subsection{Tightness across scales}

In this section we check that subsequential limits of LFPP satisfy Axiom V. For the statement, we note that we can take a subsequential limit of the joint laws of $\left(\mathrm{h}, \mathfrak{a}_{\varepsilon}^{-1} D_{\mathrm{h}}^{\varepsilon}\right)$ due to Lemma 2.5 and the Prokhorov theorem.

Lemma 2.13 Let $h$ be a whole-plane GFF normalized so that $h_{1}(0)=0$. Let $\left(h, D_{h}\right)$ be any subsequential limit of the laws of the field/metric pairs $\left(h, \mathfrak{a}_{\varepsilon}^{-1} D_{h}^{\varepsilon}\right)$. There 
are deterministic constants $\left\{\mathfrak{c}_{r}\right\}_{r \geq 0}$, depending on the law of $D_{h}$, such that the laws of the metrics $\left\{\mathfrak{c}_{r}^{-1} e^{-\xi h_{r}(0)} D_{h}(r \cdot, r \cdot)\right\}_{r>0}$ are tight w.r.t. the local uniform topology. Furthermore, the closure of this set of laws w.r.t. the Prokhorov topology for probability measures on continuous functions $\mathbb{C} \times \mathbb{C} \rightarrow[0, \infty)$ is contained in the set of laws on continuous metrics on $\mathbb{C}$. Finally, there exists $\Lambda>1$ such that for each $\delta \in(0,1)$,

$$
\Lambda^{-1} \delta^{\Lambda} \leq \frac{\mathfrak{c}_{\delta r}}{\mathfrak{c}_{r}} \leq \Lambda \delta^{-\Lambda}, \quad \forall r>0
$$

We first produce the scaling constants $\mathfrak{c}_{r}$ appearing in Axiom V.

Lemma 2.14 Consider a sequence $\mathcal{E} \subset(0,1)$ converging to zero along which $\mathfrak{a}_{\varepsilon}^{-1} D_{h}^{\varepsilon}$ converges in law to a limiting metric $D_{h}$. For each $r>0$, the limit

$$
\mathfrak{c}_{r}:=\lim _{\mathcal{E} \ni \varepsilon \rightarrow 0} \frac{r \mathfrak{a}_{\varepsilon / r}}{\mathfrak{a}_{\varepsilon}}
$$

exists and satisfies the relation (2.27) for some choice of $\Lambda>1$ depending only on $\mathcal{E}$ and $\gamma$.

Proof Let $h^{r}:=h(r \cdot)-h_{r}(0)$ be as in Lemma 2.6, so that $h^{r} \stackrel{d}{=} h$. By our choice of subsequence $\mathcal{E}$ and Lemma 2.6,

$$
\mathfrak{a}_{\varepsilon}^{-1} D_{h^{r}}^{\varepsilon / r}=r^{-1} e^{-\xi h_{r}(0)} \mathfrak{a}_{\varepsilon}^{-1} D_{h}^{\varepsilon}(r \cdot, r \cdot) \stackrel{\mathcal{E} \ni \varepsilon \rightarrow 0}{\longrightarrow} r^{-1} e^{-\xi h_{r}(0)} D_{h}(r \cdot, r \cdot)
$$

in law w.r.t. the local uniform topology on $\mathbb{C} \times \mathbb{C}$. Let $m_{r}$ be the median distance between the left and right boundaries of $[0,1]^{2}$ w.r.t. the metric on the right side of (2.29). Since $h^{r} \stackrel{d}{=} h$,

$$
\underbrace{\mathfrak{a}_{\varepsilon / r}^{-1} D_{h}^{\varepsilon / r}}_{\text {tight }} \stackrel{d}{=} \mathfrak{a}_{\varepsilon / r}^{-1} D_{h^{r}}^{\varepsilon / r}=\frac{\mathfrak{a}_{\varepsilon}}{\mathfrak{a}_{\varepsilon / r}} \underbrace{\mathfrak{a}_{\varepsilon}^{-1} D_{h^{r}}^{\varepsilon / r}}_{\begin{array}{c}
\text { convergent } \\
\text { by }(2.29)
\end{array}} .
$$

If we consider a subsequence $\mathcal{E}^{\prime}$ of $\mathcal{E}$ along which the joint law of $\mathfrak{a}_{\varepsilon / r}^{-1} D_{h}^{\varepsilon / r}$ and $\mathfrak{a}_{\varepsilon}^{-1} D_{h^{r}}^{\varepsilon / r}$ converges, then (2.30) shows that along this subsequence, $\mathfrak{a}_{\varepsilon / r} / \mathfrak{a}_{\varepsilon}$ converges to some number $s_{r}\left(\mathcal{E}^{\prime}\right)>0$ (we know the limit is strictly positive since the limits of $\mathfrak{a}_{\varepsilon / r}^{-1} D_{h}^{\varepsilon / r}$ and $\mathfrak{a}_{\varepsilon}^{-1} D_{h^{r}}^{\varepsilon / r}$ are metrics). By the definitions of $\mathfrak{a}_{\varepsilon}$ and of $m_{r}$ and Portmanteau's lemma, the median distance between the left and right boundaries of $[0,1]^{2}$ w.r.t. the metric on the left (resp. right) side of (2.30) is 1 (resp. $m_{r} / s_{r}\left(\mathcal{E}^{\prime}\right)$ ). Hence $s_{r}\left(\mathcal{E}^{\prime}\right)=m_{r}$, i.e., the limit does not depend on the choice of subsequence $\mathcal{E}^{\prime} \subset \mathcal{E}$. This shows the convergence of $\mathfrak{a}_{\varepsilon / r} / \mathfrak{a}_{\varepsilon}$ along the subsequence $\mathcal{E}$, which in turn implies the existence of the limit (2.28). The bounds (2.27) (in fact, substantially stronger bounds) are immediate from [10, Theorem 1, Equation (1.3)] and the fact the ratio of our $\mathfrak{a}_{\varepsilon}$ and the scaling factor $\lambda_{\varepsilon}$ from [10] is bounded above and below by deterministic, $\varepsilon$-independent constants (see the proof of Lemma 2.8). 
Proof of Lemma 2.13 Define $\mathfrak{c}_{r}$ for $r>0$ as in Lemma 2.14. Let $h^{r}:=h(r \cdot)-h_{r}(0)$, as in Lemma 2.6, so that $h^{r} \stackrel{d}{=} h$ and the metrics $D_{h^{r}}^{\varepsilon / r}$ and $D_{h}^{\varepsilon}$ are related as in (2.18). We know from Lemma 2.5 that the laws of the metrics $\left\{\mathfrak{a}_{\varepsilon}^{-1} D_{h}^{\varepsilon}\right\}_{0<\varepsilon<1}$ are tight, and every element of the closure of this set of laws is supported on continuous metrics on $\mathbb{C}$. It follows that the same is true for the laws of the metrics $\left\{\mathfrak{a}_{\varepsilon / r}^{-1} D_{h^{r}}^{\varepsilon / r}\right\}_{0<\varepsilon<r}$. By combining this with (2.18), we get that the laws of the metrics

$$
e^{-\xi h_{r}(0)}\left(\frac{r \mathfrak{a}_{\varepsilon / r}}{\mathfrak{a}_{\varepsilon}}\right)^{-1} \mathfrak{a}_{\varepsilon}^{-1} D_{h}^{\varepsilon}(r \cdot, r \cdot)=\mathfrak{a}_{\varepsilon / r}^{-1} D_{h^{r}}^{\varepsilon / r}, \quad \forall r>0, \quad \forall \varepsilon \in(0, r)
$$

are tight and every element of the closure of this set of laws w.r.t. the Prokhorov topology is supported on continuous metrics on $\mathbb{C}$.

Now consider a subsequence $\mathcal{E} \subset(0,1)$ along which $\left(h, \mathfrak{a}_{\varepsilon}^{-1} D_{h}^{\varepsilon}\right) \rightarrow\left(h, D_{h}\right)$ in law. By the definition (2.28) of $\mathfrak{c}_{r}$,

$$
e^{-\xi h_{r}(0)}\left(\frac{r \mathfrak{a}_{\varepsilon / r}}{\mathfrak{a}_{\varepsilon}}\right)^{-1} \mathfrak{a}_{\varepsilon}^{-1} D_{h}^{\varepsilon}(r \cdot, r \cdot) \rightarrow e^{-\xi h_{r}(0)} \mathfrak{c}_{r}^{-1} D_{h}(r \cdot, r \cdot), \quad \text { in law along } \mathcal{E}
$$

Therefore, the metrics $e^{-\xi h_{r}(0)} \mathfrak{c}_{r}^{-1} D_{h}(r \cdot r \cdot)$ for $r>0$ are all subsequential limits as $\varepsilon \rightarrow 0$ of the family of random metrics (2.31). It follows that the laws of the metrics $e^{-\xi h_{r}(0)} \mathfrak{c}_{r}^{-1} D_{h}(r \cdot, r \cdot)$ are tight and every element of the closure of this set of laws is supported on continuous metrics on $\mathbb{C}$.

\subsection{Locality}

In this section, we will prove a variant of Axiom II for subsequential limits of LFPP, restricted to the case of a whole-plane GFF (locality for a whole-plane GFF plus a continuous function will be checked in Sect. 2.6). At this point, we have not yet established that such subsequential limits can be realized as measurable functions of the field, so we will actually check a somewhat different condition. In what follows, if $K \subset \mathbb{C}$ is closed we define the $\sigma$-algebra generated by $\left.h\right|_{K}$ to be $\left.\bigcap_{\delta>0} h\right|_{B_{\delta}(K)}$. With this definition it makes sense to condition on $\left.h\right|_{K}$. The following definitions first appeared in [26].

Definition 2.15 (Local metric) Let $U \subset \mathbb{C}$ be a connected open set and let $(h, D)$ be a coupling of a GFF on $U$ and a random continuous length metric on $U$. We say that $D$ is a local metric for $h$ if for any open set $V \subset U$, the internal metric $D(\cdot, \cdot ; V)$ is conditionally independent from the pair $(h, D(\cdot, \cdot ; U \backslash \bar{V}))$ given $\left.h\right|_{\bar{V}}$.

Definition 2.15 is formulated in a slightly different way than [26, Definition 1.2]; the equivalence of the definitions is proven in [26, Lemma 2.3]. The following is [26, Definition 1.5].

Definition 2.16 (Additive local metric) Let $U \subset \mathbb{C}$ be a connected open set and let $(h, D)$ be a coupling of a GFF on $U$ and a random continuous length metric on $U$ 
which is local for $h$. For $\xi \in \mathbb{R}$, we say that $D$ is $\xi$-additive for $h$ if for each $z \in U$ and each $r>0$ such that $B_{r}(z) \subset U$, the metric $e^{-\xi h_{r}(z)} D$ is local for $h-h_{r}(z)$.

Lemma 2.17 Let $h$ be a whole-plane GFF. Let $\left(h, D_{h}\right)$ be any subsequential limit of the laws of the pairs $\left(h, \mathfrak{a}_{\varepsilon}^{-1} D_{h}^{\varepsilon}\right)$. Then $D_{h}$ is a $\xi$-additive local metric for $h$. That $i$, suppose $z \in \mathbb{C}$ and $r>0$ and that $h$ is normalized so that the circle average $h_{r}(z)$ is zero. Also let $V \subset \mathbb{C}$ be an open set. Then the internal metric $D_{h}(\cdot, \cdot ; V)$ is conditionally independent from the pair $\left(h, D_{h}(\cdot, \cdot ; \mathbb{C} \backslash \bar{V})\right)$ given $\left.h\right|_{\bar{V}}$.

There are two main difficulties in the proof of Lemma 2.17.

1. The mollified GFF $h_{\varepsilon}^{*}(z)$ of (1.1) does not exactly depend locally on $h$ (since the heat kernel $p_{\varepsilon^{2} / 2}(z, \cdot)$ does not have compact support), so the $D_{h}^{\varepsilon}$-lengths of paths are not locally determined by $h$.

2. Conditional independence does not in general behave nicely under taking limits in law.

Difficulty 1 will be resolved by means of the localization results for LFPP in Sect. 2.1. To resolve Difficulty 2, we will use the Markov property of the GFF (see Lemma 2.18) and Weyl scaling (Lemma 2.12) in order to reduce to working with metrics which are actually independent, not just conditionally independent. The use of the Markov property is the reason why we restrict to a whole-plane GFF, not a whole-plane GFF plus a bounded continuous function, in Lemma 2.17.

For the proof of Lemma 2.17 we will need the following version of the Markov property of the whole-plane GFF, which is proven in [28, Lemma 2.2]. We note that the statement of this Markov property is slightly more complicated than in the case of the zero-boundary GFF due to the need to fix the additive constant for $h$.

Lemma 2.18 ([28]) Let $z \in \mathbb{C}$ and $r>0$ and let $h$ be a whole-plane GFF with the additive constant chosen so that $h_{r}(z)=0$. For each open set $V \subset \mathbb{C}$ which is non-polar (i.e., Brownian motion started in $V$ a.s. hits $\partial V$ in finite time), we have the decomposition

$$
h=\mathfrak{h}+\stackrel{\circ}{h}
$$

where $\mathfrak{h}$ is a random distribution which is harmonic on $V$ and is determined by $\left.h\right|_{\mathbb{C} \backslash V}$ and $h$ is independent from $\mathfrak{h}$ and has the law of a zero-boundary GFF on $V$ minus its average over $\partial B_{r}(z) \cap V$. If $V$ is disjoint from $\partial B_{r}(z)$, then $h$ is a zero-boundary $G F F$ and is independent from $\left.h\right|_{\mathbb{C} \backslash V}$.

The following lemma will allow us to apply Lemma 2.18 to study $\left.h\right|_{\mathbb{C} \backslash \bar{V}}$.

Lemma 2.19 It suffices to prove Lemma 2.17 in the case when $B_{r}(z) \subset V$.

Proof Assume that we have proven Lemma 2.17 in the case when $B_{r}(z) \subset V$. Fix $z_{0} \in \mathbb{C}$ and $r_{0}>0$ such that $B_{r_{0}}\left(z_{0}\right) \subset V$ and assume that $h$ is normalized so that $h_{r_{0}}\left(z_{0}\right)=0$. By assumption, $D_{h}(\cdot, \cdot ; V)$ is conditionally independent from the pair $\left(h, D_{h}(\cdot, \cdot ; \mathbb{C} \backslash \bar{V})\right)$ given $\left.h\right|_{\bar{V}}$. 


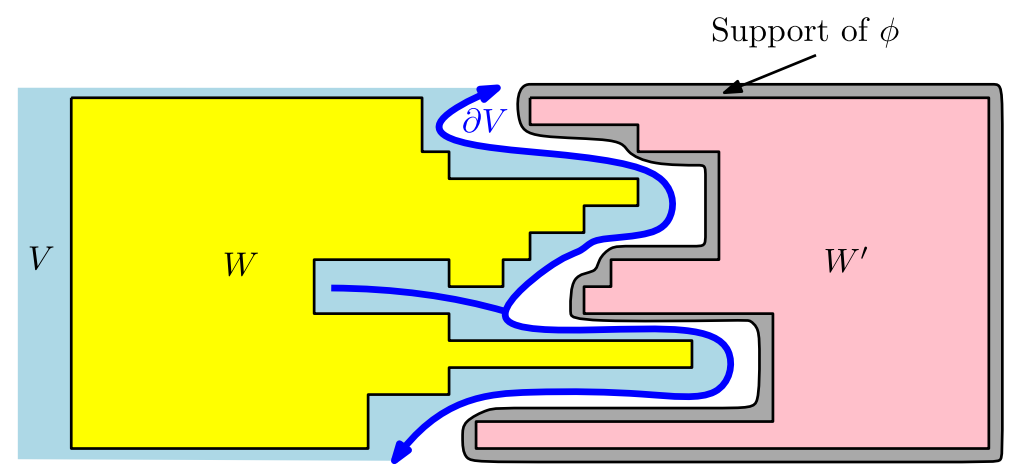

Fig. 1 Illustration of the sets used in the proof of Lemma 2.17. The set $\phi^{-1}(1)$ is not shown; it contains the closure of the pink set $W^{\prime}$ and is contained in the grey set supp $\phi$ (color figure online)

Now let $z \in \mathbb{C}$ and $r>0$ and define $\widetilde{h}:=h-h_{r}(z)$, so that $\widetilde{h}$ is a whole-plane GFF normalized so that $\widetilde{h}_{r}(z)=0$. Lemma 2.12 implies that $D_{\widetilde{h}}^{\varepsilon} \rightarrow e^{-\xi h_{r}(z)} D_{h}=: D_{\widetilde{h}}$ in law along the same subsequence for which $D_{h}^{\varepsilon} \rightarrow D_{h}$ in law, so $D_{\widetilde{h}}$ is unambiguously defined. We need to show that the conclusion of the first paragraph remains true with $\left(\widetilde{h}, D_{\widetilde{h}}\right)$ in place of $\left(h, D_{h}\right)$.

The key fact which allows us to show this is that $\widetilde{h}_{r_{0}}\left(z_{0}\right)=-h_{r}(z)$. Since $B_{r_{0}}\left(z_{0}\right) \subset$ $V$, this means that $h_{r}(z) \in \sigma\left(\left.\widetilde{h}\right|_{\bar{V}}\right)$. In particular, $\left.h\right|_{V}=\left.\widetilde{h}\right|_{\bar{V}}+h_{r}(z)$ is determined by $\left.\widetilde{h}\right|_{\bar{V}}$. Therefore, our assumption implies that $D_{h}(\cdot, \cdot ; V)$ is conditionally independent from the pair $\left(h, D_{h}(\cdot, \cdot ; \mathbb{C} \backslash \bar{V})\right)$ given $\left.\widetilde{h}\right|_{\bar{V}}$ (instead of just $\left.h\right|_{\bar{V}}$ ).

We have $D_{\widetilde{h}}(\cdot, \cdot ; V)=e^{-\xi h_{r}(z)} D_{h}(\cdot, \cdot ; V)$, so $D_{\widetilde{h}}(\cdot, \cdot ; V)$ is determined by $\left.\widetilde{h}\right|_{\bar{V}}$ and $D_{h}(\cdot, \cdot ; V)$. Similarly, $D_{\widetilde{h}}(\cdot, \cdot ; \mathbb{C} \backslash \bar{V})$ is determined by $\left.\widetilde{h}\right|_{\bar{V}}$ and $D_{h}(\cdot, \cdot ; \mathbb{C} \backslash \bar{V})$. Obviously, $h$ and $\widetilde{h}$ determine the same information. Therefore, $D_{\widetilde{h}}(\cdot, \cdot ; V)$ is conditionally independent from the pair $\left(\widetilde{h}, D_{\widetilde{h}}(\cdot, \cdot ; \mathbb{C} \backslash \bar{V})\right)$ given $\left.\widetilde{h}\right|_{\bar{V}}$, as required.

Proof of Lemma 2.17 Step 1: reductions. By Lemma 2.1, for any sequence of $\varepsilon$ 's tending to zero along which $\left(h, \mathfrak{a}_{\varepsilon}^{-1} D_{h}^{\varepsilon}\right) \rightarrow\left(h, D_{h}\right)$ in law, we also have $\left(h, \mathfrak{a}_{\varepsilon}^{-1} \widehat{D}_{h}^{\varepsilon}\right) \rightarrow$ $\left(h, D_{h}\right)$ in law. This allows us to work with $\widehat{D}_{h}^{\varepsilon}$ instead of $D_{h}^{\varepsilon}$ throughout the proof. The reason why we want to do this is the locality property (2.3) of $\widehat{D}_{h}^{\varepsilon}$.

The statement of the lemma is vacuous if $\bar{V}=\mathbb{C}$, so we can assume without loss of generality that $\bar{V} \neq \mathbb{C}$, which implies that $\mathbb{C} \backslash \bar{V}$ is non-polar. By Lemma 2.19, we can also assume without loss of generality that $B_{r}(z) \subset V$. These assumptions together with Lemma 2.18 applied with $\mathbb{C} \backslash \bar{V}$ in place of $V$ allows us to write

$$
\left.h\right|_{\mathbb{C} \backslash \bar{V}}=\mathfrak{h}+\stackrel{\circ}{h}
$$

where $\mathfrak{h}$ is a random harmonic function on $\mathbb{C} \backslash \bar{V}$ which is determined by $\left.h\right|_{\mathbb{C} \backslash \bar{V}}$ and $\stackrel{\circ}{h}$ is a zero-boundary GFF in $\mathbb{C} \backslash \bar{V}$ which is independent from $\left.h\right|_{\mathbb{C} \backslash \bar{V}}$.

Step 2: independence for LFPP. We want to apply the convergence of internal metrics given in Lemma 2.5, so we fix dyadic domains (Definition 2.4) $W, W^{\prime}$ with $\bar{W} \subset V$ and $\bar{W}^{\prime} \subset \mathbb{C} \backslash \bar{V}$ (we will eventually let $W$ and $W^{\prime}$ increase to all of $V$ and $\mathbb{C} \backslash \bar{V}$, 
respectively). Let $\phi$ be a deterministic, smooth, compactly supported bump function which is identically equal to 1 on a neighborhood of $\bar{W}^{\prime}$ and which vanishes outside of a compact subset of $\mathbb{C} \backslash \bar{V}$. See Fig. 1 for an illustration of these objects.

The restrictions of the fields $h-\phi \mathfrak{h}$ and $h$ to the set $\phi^{-1}(1) \supset \bar{W}^{\prime}$ are identical. By the locality property (2.3) of $\widehat{D}_{h}^{\varepsilon}$, if $\varepsilon>0$ is small enough that $B_{\varepsilon}\left(W^{\prime}\right) \subset \phi^{-1}(1)$, then the $\varepsilon$-LFPP metric for $h-\phi \mathfrak{h}$ satisfies

$$
\widehat{D}_{h-\phi \mathfrak{h}}^{\varepsilon}\left(\cdot, \cdot ; \bar{W}^{\prime}\right) \in \sigma(\stackrel{\circ}{h})
$$

Similarly, for small enough $\varepsilon>0$ the metric $\widehat{D}_{h}^{\varepsilon}(\cdot, \cdot ; \bar{W})$ is a.s. determined by $\left.h\right|_{V}$. Since $\left.h\right|_{V}$ and $\stackrel{\circ}{h}$ are independent, we obtain

$$
\left(\left.h\right|_{V}, \mathfrak{a}_{\varepsilon}^{-1} D_{h}^{\varepsilon}(\cdot, \cdot ; \bar{W})\right) \text { and }\left(\stackrel{\circ}{h} \mathfrak{a}_{\varepsilon}^{-1} D_{h-\phi \mathfrak{h}}^{\varepsilon}\left(\cdot, \cdot ; \bar{W}^{\prime}\right)\right) \text { are independent. }
$$

Step 3: passing to the limit. We now want to pass the independence (2.35) through to the (subsequential) scaling limit. To this end, consider a sequence $\mathcal{E}$ of positive $\varepsilon$ 's tending to zero along which $\left(h, \mathfrak{a}_{\varepsilon}^{-1} \widehat{D}_{h}^{\varepsilon}\right) \rightarrow\left(h, D_{h}\right)$ in law. By possibly passing to a further deterministic subsequence, we can arrange that in fact $\left(h, \mathfrak{h}, \mathfrak{a}_{\varepsilon}^{-1} \widehat{D}_{h}^{\varepsilon}\right) \rightarrow\left(h, \mathfrak{h}, D_{h}\right)$ in law along $\mathcal{E}$, where here the second coordinate is given the local uniform topology on $\mathbb{C} \backslash \bar{V}$. By the analog of Lemma 2.12 with $\widehat{D}^{\varepsilon}$. in place of $D^{\varepsilon}$. (which is proven in an identical manner), if we set $D_{h-\phi \mathfrak{h}}=e^{-\xi \phi \mathfrak{h}} \cdot D_{h}$, then along this same subsequence we have the convergence of joint laws

$$
\left(h, \mathfrak{h}, \mathfrak{a}_{\varepsilon}^{-1} \widehat{D}_{h}^{\varepsilon}, \mathfrak{a}_{\varepsilon}^{-1} \widehat{D}_{h-\phi \mathfrak{h}}^{\varepsilon}\right) \rightarrow\left(h, \mathfrak{h}, D_{h}, D_{h-\phi \mathfrak{h}}\right)
$$

By assertion B of Lemma 2.5, applied once to each of $h$ and $h-\phi \mathfrak{h}$, by possibly replacing $\mathcal{E}$ with a further deterministic subsequence we can find a coupling ( $\left.h, D_{h}, D_{h, W}, D_{h-\phi \mathfrak{h}, W^{\prime}}\right)$ of $\left(h, D_{h}\right)$ with length metrics on $\bar{W}$ and $\bar{W}^{\prime}$, respectively, which induce the Euclidean topology and which satisfy

$$
D_{h, W}(\cdot, \cdot ; W)=D_{h}(\cdot, \cdot ; W) \quad \text { and } \quad D_{h-\phi \mathfrak{h}, W^{\prime}}\left(\cdot, \cdot ; W^{\prime}\right)=D_{h-\phi \mathfrak{h}}\left(\cdot, \cdot ; W^{\prime}\right)
$$

such that the following is true. Along $\mathcal{E}$, we have the convergence of joint laws

$$
\begin{aligned}
& \left(h, \mathfrak{h}, \mathfrak{a}_{\varepsilon}^{-1} \widehat{D}_{h}^{\varepsilon}, \mathfrak{a}_{\varepsilon}^{-1} \widehat{D}_{h-\phi \mathfrak{h}}^{\varepsilon}, \mathfrak{a}_{\varepsilon}^{-1} \widehat{D}_{h}^{\varepsilon}(\cdot, \cdot ; \bar{W}), \mathfrak{a}_{\varepsilon}^{-1} \widehat{D}_{h-\phi \mathfrak{h}}^{\varepsilon}\left(\cdot, \cdot ; \bar{W}^{\prime}\right)\right) \\
& \quad \rightarrow\left(h,, \mathfrak{h}, D_{h}, D_{h-\phi \mathfrak{h}}, D_{h, W}, D_{h-\phi \mathfrak{h}, W^{\prime}}\right)
\end{aligned}
$$

where the last two coordinates are given the uniform topology on $\bar{W} \times \bar{W}$ and on $\bar{W}^{\prime} \times \bar{W}^{\prime}$, respectively. Since independence is preserved under convergence in law, we 
obtain from (2.35) and (2.38) that $\left(\left.h\right|_{V}, D_{h, W}\right)$ and $\left(\stackrel{\circ}{h}, D_{h-\phi \mathfrak{h}, W^{\prime}}\right)$ are independent. By (2.37), this means that

$$
\left(\left.h\right|_{V}, D_{h}(\cdot, \cdot ; W)\right) \text { and }\left(\stackrel{\circ}{h}, D_{h-\phi \mathfrak{h}}\left(\cdot, \cdot ; W^{\prime}\right)\right) \text { are independent. }
$$

Step 4: adding back in the harmonic part. By $(2.39), D_{h}(\cdot, \cdot ; W)$ is conditionally independent from $\left(\stackrel{\circ}{h}, D_{h-\phi \mathfrak{h}}\left(\cdot, \cdot ; W^{\prime}\right)\right)$ given $\left.h\right|_{V}$. We now argue that $\left(h, D_{h}\left(\cdot, \cdot ; W^{\prime}\right)\right)$ is a measurable function of $\left(\stackrel{\circ}{h}, D_{h-\phi \mathfrak{h}}\left(\cdot, \cdot ; W^{\prime}\right)\right)$ and $\left.h\right|_{V}$, so that $D_{h}(\cdot, \cdot ; W)$ is conditionally independent from $\left(h, D_{h}\left(\cdot, \cdot ; W^{\prime}\right)\right)$ given $\left.h\right|_{V}$. Indeed, by Lemma 2.12, a.s. $D_{h}\left(\cdot, \cdot ; W^{\prime}\right)=\left(e^{\xi \phi \mathfrak{h}} \cdot D_{h-\phi \mathfrak{h}}\right)\left(\cdot, \cdot ; W^{\prime}\right)$. Hence $D_{h}\left(\cdot, \cdot ; W^{\prime}\right)$ is a measurable function of $\mathfrak{h} \in \sigma\left(\left.h\right|_{\bar{V}}\right)$ and $D_{h-\phi \mathfrak{h}}\left(\cdot, \cdot ; W^{\prime}\right)$. Since $\left.h\right|_{\mathbb{C} \backslash \bar{V}}=\stackrel{\circ}{h}+\mathfrak{h}$, we get that $h$ is a measurable function of $\stackrel{\circ}{h}$ and $\left.h\right|_{\bar{V}}$. It therefore follows that $D_{h}(\cdot, \cdot ; W)$ is conditionally independent from $\left(h, D_{h}\left(\cdot, \cdot ; W^{\prime}\right)\right)$ given $\left.h\right|_{\bar{V}}$. Letting $W$ increase to $V$ and $W^{\prime}$ increase to $\mathbb{C} \backslash \bar{V}$ now concludes the proof.

\subsection{Measurability}

We have not yet established that subsequential limits of LFPP can be realized as measurable functions of the corresponding field. We will accomplish this in this subsection using a result from [26].

Lemma 2.20 Let $h$ be a whole-plane GFF normalized so that $h_{1}(0)=0$ and let $\left(h, D_{h}\right)$ be any subsequential limit of the laws of the pairs $\left(h, \mathfrak{a}_{\varepsilon}^{-1} D_{h}^{\varepsilon}\right)$. Then $D_{h}$ is a.s. determined by $h$. In particular, $\mathfrak{a}_{\varepsilon}^{-1} D_{h}^{\varepsilon} \rightarrow D_{h}$ in probability along the given subsequence.

The following theorem is a special case of [26, Corollary 1.8].

Theorem 2.21 ([26]) There is a universal constant $p \in(0,1)$ such that the following is true. Let $\xi \in \mathbb{R}$, let $h$ be a whole-plane $G F F$ normalized so that $h_{1}(0)=0$, and let $(h, D)$ be a coupling of $h$ with a random continuous length metric satisfying the following properties.

1. $D$ is a $\xi$-additive local metric for $h$ (Definition 2.16).

2. Condition on $h$ and let $D$ and $\widetilde{D}$ be conditionally i.i.d. samples from the conditional law of $D$ given $h$. There is a deterministic constant $C>0$ such that

$$
\begin{aligned}
& \mathbb{P}\left[\sup _{u, v \in \partial B_{r}(z)} \widetilde{D}\left(u, v ; B_{2 r}(z) \backslash \overline{B_{r / 2}(z)}\right) \leq C D\left(\partial B_{r / 2}(z), \partial B_{r}(z)\right)\right] \geq p \\
& \forall z \in \mathbb{C}, \quad \forall r>0 .
\end{aligned}
$$

Then $D$ is a.s. determined by $h$.

Proof of Lemma 2.20 Let $p \in(0,1)$ be as in Theorem 2.21. Lemma 2.17 implies that $D_{h}$ is a $\xi$-additive local metric for $h$. Lemma 2.13 along with the translation invariance 
of the law of $h$, modulo additive constant, implies that there exists $C>0$ (depending only on the choice of subsequence) such that for each $z \in \mathbb{C}$ and each $r>0$,

$$
\begin{aligned}
& \mathbb{P}\left[D\left(\partial B_{r / 2}(z), \partial B_{r}(z)\right) \geq C^{-1 / 2} \mathfrak{c}_{r} e^{\xi h_{r}(z)}\right] \geq \frac{1-p}{2} \text { and } \\
& \mathbb{P}\left[\sup _{u, v \in \partial B_{r}(z)} D_{h}\left(u, v ; B_{2 r}(z) \backslash \overline{B_{r / 2}(z)}\right) \leq C^{1 / 2} \mathfrak{c}_{r} e^{\xi h_{r}(z)}\right] \geq \frac{1-p}{2} .
\end{aligned}
$$

This implies that (2.40) holds for two conditionally independent samples from the conditional law of $D_{h}$ given $h$. Hence the criteria of Theorem 2.21 are satisfied, so $D_{h}$ is a.s. determined by $h$. The last statement follows from Lemma 1.3.

Proof of Theorem 1.2 Step 1: Defining a $D_{\mathrm{h}}$ for a whole-plane GFF plus a bounded continuous function. Let $h$ be a whole-plane GFF normalized so that $h_{1}(0)=0$. Lemma 2.5 implies that for any sequence of $\varepsilon$ 's tending to zero, there is a subsequence $\varepsilon_{n} \rightarrow 0$ along which $\left(h, D_{h}^{\varepsilon_{n}}\right) \rightarrow\left(h, D_{h}\right)$ in law. By Lemma $2.20, D_{h}$ is a.s. determined by $h$ and $D_{h}^{\varepsilon_{n}} \rightarrow D_{h}$ in probability. Hence every deterministic subsequence of the $\varepsilon_{n}$ 's admits a further deterministic subsequence $\varepsilon_{n_{k}}$ along which $D_{h}^{\varepsilon_{n_{k}}} \rightarrow D_{h}$ a.s. By Lemma 2.12, it is a.s. the case that for every bounded continuous function $f: \mathbb{C} \rightarrow \mathbb{R}$ simultaneously, we have $D_{h+f}^{\varepsilon_{n_{k}}} \rightarrow e^{\xi f} \cdot D_{h}$. We define $D_{h+f}:=e^{\xi f} \cdot D_{h}$. Then $D_{h+f}$ is a.s. determined by $h+f$ and $D_{h+f}^{\varepsilon_{n}}$ converges in probability to $D_{h+f}$.

This gives us a measurable function $\mathrm{h} \mapsto D_{\mathrm{h}}$ from distributions to continuous metrics on $\mathbb{C}$ which is a.s. defined whenever $\mathrm{h}$ is a whole-plane GFF plus a bounded continuous function: in particular, $D_{\mathrm{h}}$ is the a.s. limit of $D_{\mathrm{h}}^{\varepsilon_{n_{k}}}$. With this definition of $D$, Axiom I holds with $\mathrm{h}$ constrained to be a whole-plane GFF plus a bounded continuous function since we know that the limiting metric in the setting of Lemma 2.5 is a length metric. By the preceding paragraph, Axiom III holds for this definition of $D$ and with $f$ constrained to be bounded. It is immediate from the definition of LFPP that also Axiom IV holds. By Lemma 2.13, also Axiom V holds.

Step 2: locality for a whole-plane GFF plus a bounded continuous function. Axiom II in the case of a whole-plane GFF is immediate from Lemma 2.17 now that we know that $D_{h}$ is a.s. determined by $h$. We now prove Axiom II in the case when $\mathrm{h}$ is a whole-plane GFF plus a bounded continuous function. Indeed, let $V \subset \mathbb{C}$ be open and let $O \subset O^{\prime} \subset V$ be open and bounded with $\bar{O} \subset O^{\prime}$ and $\bar{O}^{\prime} \subset V$. Let $u, v \in O$ be deterministic. We will show that

$$
D_{\mathrm{h}}(u, v) \mathbb{1}_{\left\{D_{\mathrm{h}}(u, v)<D_{\mathrm{h}}\left(u, \partial O^{\prime}\right)\right\}} \in \sigma\left(\left.\mathrm{h}\right|_{V}\right) .
$$

Since $(u, v) \mapsto D_{\mathrm{h}}(u, v)$ is a.s. continuous, (2.41) implies that in fact $\left.\mathrm{h}\right|_{V}$ a.s. determines the random function $O \ni(u, v) \mapsto D_{\mathrm{h}}(u, v) \mathbb{1}_{\left\{D_{\mathrm{h}}(u, v)<D_{\mathrm{h}}\left(u, \partial O^{\prime}\right)\right\}}$. Since $\bar{O}$ is a compact subset of $O^{\prime}, O$ can be covered by finitely many sets of the form $\left\{v \in O: D_{\mathrm{h}}(u, v)<D_{\mathrm{h}}\left(u, \partial O^{\prime}\right)\right\}$ for points $u \in O$. By the definition of the internal metric $D_{\mathrm{h}}(\cdot, \cdot ; O)$, this shows that h| $\left.\right|_{V}$ a.s. determines $D_{\mathrm{h}}(\cdot, \cdot ; O)$. Letting $O$ increase to all of $V$ then shows that $\left.\mathrm{h}\right|_{V}$ a.s. determines $D_{\mathrm{h}}(\cdot, \cdot ; V)$. 
To prove (2.41), note that if we define the localized LFPP metric $\widehat{D}_{\mathrm{h}}^{\varepsilon_{n}}$ as in (2.2), then by Lemma 2.1 we have $\mathfrak{a}_{\varepsilon_{n}}^{-1} \widehat{D}_{\mathrm{h}}^{\varepsilon_{n}}(u, v) \rightarrow D_{\mathrm{h}}(u, v)$ and $\mathfrak{a}_{\varepsilon_{n}}^{-1} \widehat{D}_{\mathrm{h}}^{\varepsilon_{n}}\left(u, \partial O^{\prime}\right) \rightarrow$ $D_{\mathrm{h}}\left(u, \partial O^{\prime}\right)$ in probability. Therefore,

$\mathfrak{a}_{\varepsilon_{n}}^{-1} \widehat{D}_{\mathrm{h}}^{\varepsilon_{n}}(u, v) \mathbb{1}_{\left\{\widehat{D}_{\mathrm{h}}^{\varepsilon_{n}}(u, v)<\widehat{D}_{\mathrm{h}}^{\varepsilon_{n}}\left(u, \partial O^{\prime}\right)\right\}} \rightarrow D_{\mathrm{h}}(u, v) \mathbb{1}_{\left\{D_{\mathrm{h}}(u, v)<D_{\mathrm{h}}\left(u, \partial O^{\prime}\right)\right\}}, \quad$ in probability.

By (2.3) and since $\bar{O}^{\prime} \subset V$, the random variable on the left side of (2.42) is a.s. determined by $\left.\mathrm{h}\right|_{V}$ for large enough $n \in \mathbb{N}$. Thus (2.41) holds.

Step 3: extending to unbounded continuous function. We will now extend the definition of $D$ to the case of a whole-plane GFF plus an unbounded continuous function and check that the axioms remain true. To this end, let $h$ be a whole-plane GFF and let $f$ be a possibly random unbounded continuous function. If $V \subset \mathbb{C}$ is open and bounded and $\phi$ is a smooth compactly supported bump function which is identically equal to 1 on $V$, then $\phi f$ is bounded so we can define the metric $D_{h+f}^{V}:=D_{h+\phi f}(\cdot, \cdot ; V)$. By Axiom II in the case of a whole-plane GFF plus a bounded continuous function, this metric is a.s. determined by $\left.(h+\phi f)\right|_{V}=\left.(h+f)\right|_{V}$, in a manner which does not depend on $\phi$. We now define the $D_{h+f}$-length of any continuous path $P$ in $\mathbb{C}$ to be the $D_{h+f}^{V}$-length of $P$, where $V \subset \mathbb{C}$ is a bounded open set which contains $P$ The definition does not depend on the choice of $V$. We define $D_{h+f}(z, w)$ for $z, w \in \mathbb{C}$ to be the infimum of the $D_{h+f}$-lengths of continuous paths from $z$ to $w$. Then $D_{h+f}$ is a length metric on $\mathbb{C}$ which is a.s. determined by $D_{h+f}$ and which satisfies $D_{h+f}(\cdot, \cdot ; V)=D_{h+f}^{V}$ for each bounded open set $V \subset \mathbb{C}$.

With the above definition, it is immediate from the case of a whole-plane GFF plus a bounded continuous function that the axioms in the definition of a weak $\gamma$-LQG metric are satisfied to the mapping $\mathrm{h} \mapsto D_{\mathrm{h}}$, which is a.s. defined whenever $\mathrm{h}$ is a whole-plane GFF plus a continuous function.

\section{Proofs of quantitative properties of weak LQG metrics}

In this section we will prove the estimates stated in Sect. 1.3. Actually, in many cases we will prove a priori stronger estimates which are required to be uniform across different Euclidean scales. With what we know now, these estimates are not implied by the estimates stated in Sect. 1.3 since we are working with a weak $\gamma$-LQG metric so we have tightness across scales instead of exact scale invariance. However, $a$ posteriori, once it is proven that a weak $\gamma$-LQG metric satisfies the coordinate change formula (1.9) (which will be done in [25], building on the results in the present paper), the estimates in this section are equivalent to the estimates in Sect. 1.3. Throughout this section, $D$ denotes a weak LQG metric and $h$ denotes a whole-plane GFF normalized so that $h_{1}(0)=0$. 

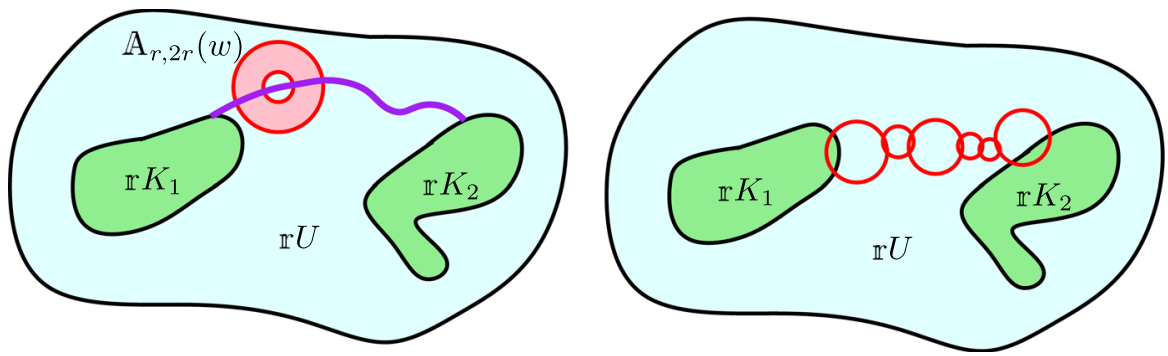

Fig. 2 Left: To prove the lower bound in Proposition 3.1, we cover $r U$ by balls $B_{r / 2}(w)$ such that the $D_{h}$-distance across the annulus $\mathbb{A}_{r, 2 r}(w)$ is bounded below. Each path from $\mathbb{r} K_{1}$ to $\mathbb{r}\left(K_{2} \cup \partial U\right)$ must cross at least one of these annuli (one such path is shown in purple). Right: To prove the upper bound in Proposition 3.1, we cover $\mathrm{r} U$ by balls $B_{r / 2}(w)$ for which the $D_{h}$-diameter of the circle $\partial B_{r}(w)$ is bounded above, then string together a path of such circles from $K_{1}$ to $K_{2}$ (color figure online)

\subsection{Estimate for the distance between sets}

The goal of this subsection is to prove the following more precise version of Theorem 1.9 which is required to be uniform across scales. For the statement, we recall the scaling constants $\mathfrak{c}_{r}$ for $r>0$ from Axiom $\mathrm{V}$.

Proposition 3.1 Let $U \subset \mathbb{C}$ be an open set (possibly all of $\mathbb{C}$ ) and let $K_{1}, K_{2} \subset U$ be connected, disjoint compact sets which are not singletons. For each $\mathbb{r}>0$, it holds with superpolynomially high probability as $A \rightarrow \infty$, at a rate which is uniform in the choice of $\mathbb{r}$, that

$$
A^{-1} \mathfrak{c}_{\mathbb{r}} e^{\xi h_{\mathrm{r}}(0)} \leq D_{h}\left(\mathbb{r} K_{1}, \mathrm{r} K_{2} ; \mathrm{r} U\right) \leq A \mathfrak{c}_{\mathbb{r}} e^{\xi h_{\mathrm{r}}(0)}
$$

We now explain the idea of the proof of Proposition 3.1; see Fig. 2 for an illustration. Using Axiom V and a general "local independence" lemma for the GFF (see Lemma 3.3), we can, with extremely high probability, cover $\mathbb{r} U$ by small Euclidean balls $B_{r / 2}(w)$ such that $r \in\left[\varepsilon^{2} \mathbb{r}, \varepsilon \mathbb{r}\right]$ and the $D_{h}$-distance across the annulus $\mathbb{A}_{r, 2 r}(w)$ is bounded below by a constant times $\mathfrak{c}_{r} e^{\xi h_{r}(w)}$. Any path from $\mathbb{r} K_{1}$ to $\mathbb{r} K_{2}$ must cross at least one of these annuli. This leads to a lower bound for $D_{h}\left(\mathbb{r} K_{1}, \mathbb{r} K_{2} ; \mathbb{r} U\right)$ in terms of

$$
\inf _{r \in\left[\varepsilon^{2} \mathbb{r}, \varepsilon \mathbb{r}\right]} \mathfrak{c}_{r} \quad \text { and } \inf _{r \in\left[\varepsilon^{2} \mathbb{r}, \varepsilon \mathbb{r}\right]} \inf _{w \in \mathbb{r} U} e^{\xi h_{r}(w)} .
$$

The first infimum in (3.2) can be bounded below by a positive power of $\varepsilon$ times $\mathfrak{c}_{\mathbb{r}}$ by (1.8). By being a little more careful about how we choose the balls $B_{r / 2}(w)$, the second term in (3.2) can be reduced to an infimum over finitely many values of $r$ and $w$, which can then be bounded below by a positive power of $\varepsilon$ times $e^{\xi h_{\mathrm{r}}(0)}$ using the Gaussian tail bound and a union bound (see Lemma 3.4). Choosing $\varepsilon$ to be an appropriate power of $A$ then concludes the proof.

The upper bound in (3.1) is proven similarly, but in this case we instead cover $U$ by balls $B_{r / 2}(w)$ for which the $D_{h}$-diameter of the circle $\partial B_{r}(w)$ is bounded above 
by a constant times $\mathfrak{c}_{r} e^{\xi h_{r}(w)}$, then "string together" a collection of such circles to get a path from $\mathrm{r} K_{1}$ to $\mathrm{r} K_{2}$ whose $D_{h}$-length is bounded above. The hypothesis that $K_{1}$ and $K_{2}$ are connected and are not singletons allows us to force some of the circles in this path to intersect $K_{1}$ and $K_{2}$.

We now explain how to cover $U$ by Euclidean balls with the desired properties. For $C>1, z \in \mathbb{C}$, and $r>0$, let $E_{r}(z ; C)$ be the event that

$$
\sup _{u, v \in \partial B_{r}(z)} D_{h}\left(u, v ; \mathbb{A}_{r / 2,2 r}(z)\right) \leq C \mathfrak{c}_{r} e^{\xi h_{r}(0)} \quad \text { and } \quad D_{h}\left(\partial B_{r}(z), \partial B_{2 r}(z)\right) \geq C^{-1} \mathfrak{c}_{r} e^{\xi h_{r}(0)}
$$

Lemma 3.2 For each $v>0$ and each $M>0$, there exists $C=C(v, M)>1$ such that for each $\mathrm{r}>0$, it holds with probability at least $1-O_{\varepsilon}\left(\varepsilon^{M}\right)$ as $\varepsilon \rightarrow 0$, at a rate which is uniform in $\mathbb{r}$, that the following is true. For each $z \in B_{\mathrm{r} \varepsilon^{-M}}(0)$, there exists

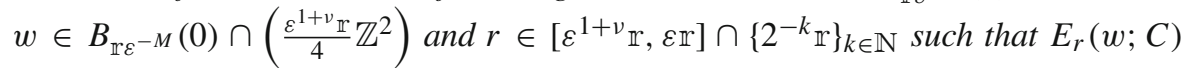
occurs and $z \in B_{\mathrm{r} \varepsilon}^{1+v} / 2(w)$.

We will prove Lemma 3.2 using the following result from [26], which in turn follows from the near-independence of the GFF across disjoint concentric annuli. See in particular [26, Lemma 3.1].

Lemma 3.3 Fix $0<s_{1}<s_{2}<1$. Let $\left\{r_{k}\right\}_{k \in \mathbb{N}}$ be a decreasing sequence of positive numbers such that $r_{k+1} / r_{k} \leq s_{1}$ for each $k \in \mathbb{N}$ and let $\left\{E_{r_{k}}\right\}_{k \in \mathbb{N}}$ be events such that $E_{r_{k}} \in \sigma\left(\left.\left(h-h_{r_{k}}(0)\right)\right|_{\mathbb{A}_{s_{1} r_{k}, s_{2} r_{k}}(0)}\right)$ for each $k \in \mathbb{N}$. For $K \in \mathbb{N}$, let $N(K)$ be the number of $k \in[1, K]_{\mathbb{Z}}$ for which $E_{r_{k}}$ occurs.

For each $a>0$ and each $b \in(0,1)$, there exists $p=p\left(a, b, s_{1}, s_{2}\right) \in(0,1)$ and $c=c\left(a, b, s_{1}, s_{2}\right)>0$ such that if

$$
\mathbb{P}\left[E_{r_{k}}\right] \geq p, \quad \forall k \in \mathbb{N},
$$

then

$$
\mathbb{P}[N(K)<b K] \leq c e^{-a K}, \quad \forall K \in \mathbb{N}
$$

Proof of Lemma 3.2 By Axioms IV and V [also see (1.10)], for each $p \in(0,1)$ there exists $C>1$ such that for every $z \in \mathbb{C}$ and $r>0, \mathbb{P}\left[E_{r}(z ; C)\right] \geq p$. By the locality of $D_{h}$ and Axiom III, the event $E_{r}(z ; C)$ is determined by $\left.\left(h-h_{3 r}(z)\right)\right|_{\mathbb{A}_{r / 2,2 r}(z)}$. We can therefore apply Lemma 3.3 to a logarithmic (in $\varepsilon$ ) number of values of $r \in$ $\left[\varepsilon^{1+v} \mathbb{r}, \varepsilon \mathbb{r}\right] \cap\left\{2^{-k_{\mathbb{r}}}\right\}_{k \in \mathbb{N}}$ to find that for any choice of $v>1$ and $\widetilde{M}>0$, there is a large enough $C=C(\nu, \widetilde{M})>1$ such that the following is true. For each $z \in \mathbb{C}$ it holds with probability at least $1-O_{\varepsilon}\left(\varepsilon^{\tilde{M}}\right)$ that $E_{r}(z ; C)$ occurs for at least one value of $r \in\left[\varepsilon^{1+v}, \varepsilon \mathbb{r}\right] \cap\left\{2^{-k} \mathbb{r}\right\}_{k \in \mathbb{N}}$. We now conclude the proof by choosing $\tilde{M}$ to be sufficiently large, in a manner depending only on $v, M$, and taking a union bound over all $z \in B_{\mathbb{r} \varepsilon^{-M}}(0) \cap\left(\frac{\varepsilon^{1+v} \mathbb{r}}{4} \mathbb{Z}^{2}\right)$. 
The occurrence of the event $E_{r}(z ; C)$ allows us to bound distances in terms of circle averages and the scaling coefficients $\mathfrak{c}_{r}$. The $\mathfrak{c}_{r}$ 's can be bounded using (1.8). To bound the circle averages, we will need the following lemma.

Lemma 3.4 For each $v>0$, each $q>2+2 v$, each $R>0$, and each $\mathrm{r}>0$, it holds with probability $1-O_{\varepsilon}\left(\varepsilon^{\frac{q^{2}}{2(1+\sqrt{v})^{2}}-2-2 v}\right)$, at a rate depending only on $q$ and $R$ (not on $\mathbb{1})$ that

$$
\sup \left\{\left|h_{r}(w)-h_{\mathbb{r}}(0)\right|: w \in B_{R \mathbb{r}}(0) \cap\left(\frac{\varepsilon^{1+v_{\mathbb{r}}}}{4} \mathbb{Z}^{2}\right), r \in\left[\varepsilon^{\left.1+v_{\mathbb{r}}, \varepsilon \mathbb{r}\right]}\right\} \leq q \log \varepsilon^{-1} .\right.
$$

Proof Fix $s \in(0, q)$ to be chosen momentarily. For each $w \in B_{R \mathrm{r}}(0)$, the random variable $t \mapsto h_{e^{-t} \varepsilon \mathrm{r}}(w)-h_{\varepsilon \mathrm{r}}(w)$ is a standard linear Brownian motion [16, Section 3]. We can therefore apply the Gaussian tail bound to find that

$$
\mathbb{P}\left[\sup _{r \in\left[\varepsilon^{\left.1+v_{\mathbb{r}, \varepsilon \mathbb{r}}\right]}\right.}\left|h_{r}(w)-h_{\varepsilon \mathbb{r}}(w)\right| \leq s \log \varepsilon^{-1}\right] \geq 1-O_{\varepsilon}\left(\varepsilon^{s^{2} /(2 v)}\right) .
$$

The random variables $h_{\varepsilon \mathrm{r}}(w)-h_{\mathrm{r}}(0)$ for $w \in B_{R \mathrm{r}}(0)$ are centered Gaussian with variance $\log \varepsilon^{-1}+O_{\varepsilon}(1)$. Applying the Gaussian tail bound again therefore gives

$$
\mathbb{P}\left[\left|h_{\varepsilon \mathbb{r}}(w)-h_{\mathbb{r}}(0)\right| \leq(q-s) \log \varepsilon^{-1}\right] \geq 1-O_{\varepsilon}\left(\varepsilon^{(q-s)^{2} / 2}\right) .
$$

Combining (3.7) and (3.8) applied with $s=q \sqrt{v} /(1+\sqrt{v})$ shows that for $w \in$ $B_{R \mathbb{r}}(0)$,

$$
\mathbb{P}\left[\sup _{r \in\left[\varepsilon^{\left.1+v_{\mathbb{r}, \varepsilon \mathbb{r}}\right]}\right.}\left|h_{r}(w)-h_{\mathbb{r}}(0)\right| \leq q \log \varepsilon^{-1}\right] \geq 1-O_{\varepsilon}\left(\varepsilon^{\frac{q^{2}}{2(1+\sqrt{v})^{2}}}\right) .
$$

We now conclude by means of a union bound over $O_{\varepsilon}\left(\varepsilon^{-2-2 v}\right)$ values of $w \in B_{R \mathbb{r}}(0) \cap$ $\left(\frac{\varepsilon^{1+v}}{4} \mathbb{Z}^{2}\right)$.

Proof of Proposition 3.1 Throughout the proof, all $O(\cdot)$ and $o(\cdot)$ errors are required to be uniform in the choice of $r$. We also impose the requirement that $U$ is bounded-we will explain at the very end of the proof how to get rid of this requirement.

Set $v=1$, say, and fix a large $M>1$, which we will eventually send to $\infty$. Let $C=C(1, M)>1$ be chosen as in Lemma 3.2 and for $\varepsilon \in(0,1)$ and $\mathbb{r}>0$, let $F_{\mathrm{r}}^{\varepsilon}$ be the event of Lemma 3.2 for this choice of $v, M, C$, so that $\mathbb{P}\left[F_{\text {r }}^{\varepsilon}\right]=1-O_{\varepsilon}\left(\varepsilon^{M}\right)$. We will eventually take $\varepsilon=A^{-b / \sqrt{M}}$ for a small constant $b>0$, so $\varepsilon^{M}$ will be a large negative power of $A$ (i.e., the power goes to $\infty$ as $M \rightarrow \infty$ ) but $\varepsilon^{\sqrt{M}}$ will be a fixed negative power of $A$ (which does not go to $\infty$ when $M \rightarrow \infty$ ). 
By Lemma 3.4 (applied with $v=1$ and $q=2 \sqrt{2} \sqrt{4+M}$ ), it holds with probability $1-O_{\varepsilon}\left(\varepsilon^{M}\right)$ that

$$
\sup \left\{\left|h_{r}(w)-h_{\mathbb{r}}(0)\right|: w \in B_{\mathbb{r}}(\mathbb{r} U) \cap\left(\frac{\varepsilon^{2} \mathbb{r}}{4} \mathbb{Z}^{2}\right), r \in\left[\varepsilon^{2} \mathrm{r}, \varepsilon \mathbb{r}\right]\right\} \leq 2 \sqrt{2} \sqrt{4+M} \log \varepsilon^{-1} .
$$

Henceforth assume that $F_{\mathrm{r}}^{\varepsilon}$ occurs and (3.10) holds, which happens with probability $1-O_{\varepsilon}\left(\varepsilon^{M}\right)$. We will now prove lower and upper bounds for $D_{h}\left(\mathrm{r} K_{1}, \mathrm{r} K_{2} ; \mathrm{r} U\right)$ in terms of $\varepsilon$.

Step 1: lower bound. By the definition of $F_{\mathrm{r}}^{\varepsilon}$, if $\varepsilon$ is sufficiently small, depending on $K_{1}, K_{2}, U$, then each path from $\mathbb{r} K_{1}$ to $\mathbb{r}\left(K_{2} \cup \partial U\right)$ must cross from $\partial B_{r}(w)$ to $\partial B_{2 r}(w)$ for some $w \in B_{\varepsilon \mathbb{r}}(\mathbb{r} U) \cap\left(\frac{\varepsilon^{2} \mathbb{r}}{4} \mathbb{Z}^{2}\right)$ and $r \in\left[\varepsilon^{2} \mathbb{r}, \varepsilon \mathbb{r}\right] \cap\left\{2^{-k} \mathbb{r}\right\}_{k \in \mathbb{N}}$ for which $E_{r}(w ; C)$ occurs. Therefore,

$$
\begin{aligned}
& D_{h}\left(\mathbb{r} K_{1}, \mathbb{r} K_{2}\right) \\
& \geq \inf \left\{C^{-1} \mathfrak{c}_{r} e^{\xi h_{r}(w)}: w \in B_{\varepsilon \mathbb{r}}(\mathbb{r} U) \cap\left(\frac{\varepsilon^{2} \mathbb{r}}{4} \mathbb{Z}^{2}\right), r \in\left[\varepsilon^{2} \mathbb{r}, \varepsilon \mathbb{r}\right]\right\} \\
& \geq C^{-1} \varepsilon^{\xi 2 \sqrt{2} \sqrt{4+M}} e^{\xi h_{\mathbb{r}}(0)} \inf \left\{\mathfrak{c}_{r}: r \in\left[\varepsilon^{2} \mathbb{r}, \varepsilon \mathbb{r}\right]\right\} \quad(\text { by }(3.10)) \\
& \geq \Lambda^{-1} \varepsilon^{\xi 2 \sqrt{2} \sqrt{4+M}+2 \Lambda+o_{\varepsilon}(1)} \mathfrak{c}_{\mathbb{r}} e^{\xi h_{\mathbb{r}}(0)} \quad(\text { by }(1.8)) .
\end{aligned}
$$

Step 2: upper bound. It is easily seen from the definition of $F_{\mathrm{r}}^{\varepsilon}$ (see Lemma 3.5) that if $\varepsilon$ is sufficiently small (depending only on $K_{1}, K_{2}$, and $U$ ) then the union of the circles $\partial B_{r}(w)$ for $w \in B_{\varepsilon \mathbb{r}}(\mathbb{r} U) \cap\left(\frac{\varepsilon^{2} \mathbb{r}}{4} \mathbb{Z}^{2}\right)$ and $r \in\left[\varepsilon^{2} \mathbb{r}, \varepsilon \mathbb{r}\right] \cap\left\{2^{-k_{\mathbb{r}}}\right\}_{k \in \mathbb{N}}$ such that $E_{r}(w ; C)$ occurs contains a path from $\mathrm{r} K_{1}$ to $\mathrm{r} K_{2}$ which is contained in $\mathrm{r} U$. The total number of such circles is at most $\varepsilon^{-4-o_{\varepsilon}(1)}$, so by the triangle inequality,

$$
\begin{aligned}
& D_{h}\left(\mathbb{r} K_{1}, \mathbb{r} K_{2} ; \mathbb{r} U\right) \\
& \quad \leq \varepsilon^{-4-o_{\varepsilon}(1)} \sup \left\{C \mathfrak{c}_{r} e^{\xi h_{r}(w)}: w \in B_{\varepsilon \mathbb{r}}(\mathbb{r} U) \cap\left(\frac{\varepsilon^{2} \mathbb{r}}{4} \mathbb{Z}^{2}\right), r \in\left[\varepsilon^{2} \mathbb{r}, \varepsilon \mathbb{r}\right]\right\} \\
& \leq \varepsilon^{-4-\xi 2 \sqrt{2} \sqrt{4+M}-o_{\varepsilon}(1)} e^{\xi h_{\mathbb{r}}(0)} \sup \left\{\mathfrak{c}_{r}: r \in\left[\varepsilon^{2} \mathbb{r}, \varepsilon \mathbb{r}\right]\right\} \quad \text { (by (3.10)) } \\
& \leq \Lambda \varepsilon^{-4-\xi 2 \sqrt{2} \sqrt{4+M}-2 \Lambda-o_{\varepsilon}(1)} \mathfrak{c}_{\mathbb{r}} e^{\xi h_{\mathbb{r}}(0)} \quad \text { (by (1.8)). }
\end{aligned}
$$

Step 3: Choosing $\varepsilon$. The bounds (3.11) and (3.12) hold with probability $1-O_{\varepsilon}\left(\varepsilon^{M}\right)$. Given $A>0$, we now choose $\varepsilon=A^{-b / \sqrt{M}}$, where $b>0$ is a small constant (depending only on $\xi, \Lambda$ ) chosen so that the right side of (3.11) is at least $A^{-1} \mathfrak{c}_{\mathbb{r}} e^{\xi h_{\mathbb{r}}}(0)$ and the right side of (3.12) is at most $A \mathfrak{c}_{\mathbb{r}} e^{\xi h_{\mathbb{r}}(0)}$. Then (3.11) and (3.12) imply that

$$
\mathbb{P}\left[D_{h}\left(\mathrm{r} K_{1}, \mathrm{r} K_{2}\right) \geq A^{-1} \mathfrak{c}_{\mathbb{r}} e^{\xi h_{\mathrm{r}}(0)}, D_{h}\left(\mathrm{r} K_{1}, \mathrm{r} K_{2} ; \mathrm{r} U\right) \leq \mathfrak{c}_{\mathbb{r}} e^{\xi h_{\mathrm{r}}(0)}\right] \geq 1-O_{A}\left(A^{-b \sqrt{M}}\right)
$$


If $U^{\prime}$ is a possibly unbounded open subset of $\mathbb{C}$ with $U \subset U^{\prime}$, then $D_{h}\left(\mathbb{r} K_{1}, \mathbb{r} K_{2}\right) \leq$ $D_{h}\left(\mathrm{r} K_{1}, \mathrm{r} K_{2} ; \mathrm{r} U^{\prime}\right) \leq D_{h}\left(K_{1}, K_{2} ; \mathrm{r} U\right)$. Since $M$ can be made arbitrarily large, we now obtain (3.1) (with $U$ possibly unbounded) from (3.13).

The following lemma was used in the proof of the upper bound of Proposition 3.1.

Lemma 3.5 Assume that we are in the setting of Proposition 3.1, with $U$ bounded. Define the event $F_{\mathrm{r}}^{\varepsilon}$ as in the proof of Proposition 3.1. For small enough $\varepsilon>0$ (depending on $\left.K_{1}, K_{2}, U\right)$, on $F_{\mathbb{r}}^{\varepsilon}$, the union of the circles $\partial B_{r}(w)$ for $w \in B_{\varepsilon \mathrm{r}}(\mathbb{r} U) \cap$ $\left(\frac{\varepsilon^{2} \mathbb{r}}{4} \mathbb{Z}^{2}\right)$ and $r \in\left[\varepsilon^{2} \mathbb{r}, \varepsilon \mathbb{r}\right] \cap\left\{2^{-k} \mathbb{r}\right\}_{k \in \mathbb{N}}$ such that $E_{r}(w ; C)$ occurs contains a path from $\mathbb{r} K_{1}$ to $\mathbb{r} K_{2}$ which is contained in $\mathbb{r} U$.

Proof Throughout the proof we assume that $F_{\mathrm{r}}^{\varepsilon}$ occurs. By the definition of $F_{\mathrm{r}}^{\varepsilon}$ and since $U$ is connected, if $\varepsilon$ is chosen so be sufficiently small then the union of the balls $B_{r}(w)$ for $w, r$ as in the lemma statement contains a path from $\mathbb{r} K_{1}$ to $K_{2}$ which is contained in $U$. Let $\mathcal{B}$ be a sub-collection of these balls which is minimal in the sense that $\bigcup_{B \in \mathcal{B}} B$ contains a path from $\mathbb{r} K_{1}$ to $\mathbb{r} K_{2}$ in $\mathbb{r} U$ and no proper sub-collection of the balls in $\mathcal{B}$ has this property. Choose a path $P$ from $\mathbb{r} K_{1}$ to $\mathbb{r} K_{2}$ in $(\mathbb{r} U) \cap \bigcup_{B \in \mathcal{B}} B$.

We first observe that $\bigcup_{B \in \mathcal{B}} B$ is connected. Indeed, if this set had two proper disjoint open subsets, then each would have to intersect $P$ (by minimality) which would contradict the connectedness of $P$. Furthermore, by minimality, no ball in $\mathcal{B}$ is properly contained in another ball in $\mathcal{B}$.

We claim that $\bigcup_{B \in \mathcal{B}} \partial B$ is connected. Indeed, if this were not the case then we could partition $\mathcal{B}=\mathcal{B}_{1} \sqcup \mathcal{B}_{2}$ such that $\mathcal{B}_{1}$ and $\mathcal{B}_{2}$ are non-empty and $\bigcup_{B \in \mathcal{B}_{1}} \partial B$ and $\bigcup_{B \in \mathcal{B}_{2}} \partial B$ are disjoint. By the minimality of $\mathcal{B}$, it cannot be the case that any ball in $\mathcal{B}_{2}$ is contained in $\bigcup_{B \in \mathcal{B}_{1}} B$. Furthermore, since $\bigcup_{B \in \mathcal{B}_{1}} \partial B$ and $\bigcup_{B \in \mathcal{B}_{2}} \partial B$ are disjoint, it cannot be the case that any ball in $\mathcal{B}_{2}$ intersects both $\bigcup_{B \in \mathcal{B}_{1}} B$ and $\mathbb{C} \backslash \bigcup_{B \in \mathcal{B}_{1}} B$ (otherwise, such a ball would have to intersect the boundary of some ball in $\mathcal{B}_{1}$ ). Therefore, $\bigcup_{B \in \mathcal{B}_{1}} B$ and $\bigcup_{B \in \mathcal{B}_{2}} \partial B$ are disjoint. Since no element of $\mathcal{B}_{1}$ can be contained in $\bigcup_{B \in \mathcal{B}_{2}} B$, we get that $\bigcup_{B \in \mathcal{B}_{1}} B$ and $\bigcup_{B \in \mathcal{B}_{2}} B$ are disjoint. This contradicts the connectedness of $\bigcup_{B \in \mathcal{B}} B$, and therefore gives our claim.

Since $P$ is a path from $\mathbb{r} K_{1}$ to $\mathrm{r} K_{2}$ and each of $\mathrm{r} K_{1}$ and $\mathrm{r} K_{2}$ is connected and not a single point, if $\varepsilon<\frac{1}{2}\left(\operatorname{diam}\left(K_{1}\right) \wedge \operatorname{diam}\left(K_{2}\right)\right)$, then the boundaries of the balls in $\mathcal{B}$ which contain the starting and endpoint points of $P$ must intersect $K_{1}$ and $K_{2}$, respectively. Hence for such an $\varepsilon, \bigcup_{B \in \mathcal{B}} \partial B$ contains a path from $\mathbb{r} K_{1}$ to $\mathbb{r} K_{2}$, as required.

\subsection{Asymptotics of the scaling constants}

The goal of this section is to prove Theorem 1.5. We will accomplish this by comparing $D_{h}$-distances to a variant of the Liouville first passage percolation (LFPP) which we now define.

For $\varepsilon \in(0,1)$ and $U \subset \mathbb{C}$, we view $U \cap\left(\varepsilon \mathbb{Z}^{2}\right)$ as a graph with adjacency defined by 
$z, w \in U \cap\left(\varepsilon \mathbb{Z}^{2}\right)$ are connected by an edge if and only if $|z-w| \in\{\varepsilon, \sqrt{2} \varepsilon\}$.

Note that this differs from the standard nearest-neighbor graph structure in that we also include the diagonal edges. We define the discretized $\varepsilon$-LFPP metric with parameter $\xi$ on $U$ by

$$
\widetilde{D}_{h}^{\varepsilon}(z, w ; U):=\min _{\pi: z \rightarrow w} \sum_{j=0}^{|\pi|} e^{\xi h_{\varepsilon}(\pi(j))}, \quad \forall z, w \in U \cap\left(\varepsilon \mathbb{Z}^{2}\right),
$$

where the minimum is over all paths $\pi:[0,|\pi|]_{\mathbb{Z}} \rightarrow U \cap\left(\varepsilon \mathbb{Z}^{2}\right)$ from $z$ to $w$ in $U \cap\left(\varepsilon \mathbb{Z}^{2}\right)$ [the tilde is to distinguish this from the variant of LFPP defined in (1.5)].

Recall that $\mathbb{S}=(0,1)^{2}$ denotes the open Euclidean unit square. Below, we will show, using Proposition 3.1 and a union bound over a polynomial number of $\delta \mathrm{r} \times \delta \mathrm{r}$ squares contained in $\mathbb{r} \mathbb{S}$, that with high probability,

$$
\mathfrak{c}_{\mathbb{r}}=\delta^{o_{\delta}(1)} \mathfrak{c}_{\delta \mathrm{r}} \times\left(\widetilde{D}_{h}^{\delta \mathrm{r}} \text { distance between two sides of } \mathrm{r} \mathbb{S}\right) .
$$

The reason why discretized LFPP comes up in this estimate is the circle average term $e^{\xi h_{\mathrm{r}}(0)}$ in Proposition 3.1. We know that the $\widetilde{D}_{h}^{\delta \mathrm{r}}$ distance across the square $\mathrm{r} \mathbb{S}$ is of order $\delta^{-\xi Q+o_{\delta}(1)}$, uniformly in $\mathbb{r}$, by the results of [12] (see Lemma 3.6). Hence (3.16) leads to $\mathfrak{c}_{\delta \mathrm{r}}=\delta^{\xi Q+o_{\delta}(1)} \mathfrak{c}_{\mathrm{r}}$, as required.

For a square $S \subset \mathbb{C}$, we write $\partial_{\mathrm{L}}^{\varepsilon} S$ and $\partial_{\mathrm{R}}^{\varepsilon} S$ for the set of leftmost (resp. rightmost) vertices of $S \cap\left(\varepsilon \mathbb{Z}^{2}\right)$.

Lemma 3.6 Fix $\zeta \in(0,1)$. For $\mathbb{1}>0$, it holds with probability tending to 1 as $\delta \rightarrow 0$, uniformly in the choice of $\mathrm{r}$, that

$$
\widetilde{D}_{h}^{\delta \mathrm{r}}\left(\partial_{\mathrm{L}}^{\delta \mathrm{r}}(\mathrm{r} \mathbb{S}), \partial_{\mathrm{R}}^{\delta \mathrm{r}}(\mathrm{r} \mathbb{S}) ; \mathrm{r} \mathbb{S}\right) \in\left[\delta^{-\xi Q+\zeta} e^{\xi h_{\mathrm{r}}(0)}, \delta^{-\xi Q-\zeta} e^{\xi h_{\mathrm{r}}(0)}\right] .
$$

Proof We first reduce to the case when $\mathrm{r}=1$. Indeed, by the scale and translation invariance of the law of $h$, modulo additive constant, we have $h(\mathbb{r} \cdot)-h_{\mathrm{r}}(0) \stackrel{d}{=} h$. Moreover, from the definition (3.15) it is easily seen that

$$
\widetilde{D}_{h(\mathrm{r} \cdot)-h_{\mathbb{r}}(0)}^{\delta}(\cdot, \cdot ; \mathbb{S})=e^{-\xi h_{\mathbb{r}}(0)} \widetilde{D}_{h}^{\delta \mathrm{r}}(\cdot, \cdot ; \mathrm{r} \mathbb{S}) .
$$

Hence $e^{-\xi h_{\mathrm{r}}(0)} \widetilde{D}_{h}^{\delta \mathrm{r}}(\cdot, \cdot ; \mathrm{r} \mathbb{S}) \stackrel{d}{=} \widetilde{D}_{h}^{\delta}(\cdot, \cdot ; \mathbb{S})$, so we only need to prove the lemma when $\mathbb{r}=1$, i.e., we need to show that with probability tending to 1 as $\delta \rightarrow 0$, we have

$$
\widetilde{D}_{h}^{\delta}\left(\partial_{\mathrm{L}}^{\delta} \mathbb{S}, \partial_{\mathrm{R}}^{\delta} \mathbb{S} ; \mathbb{S}\right)=\delta^{-\xi Q+o_{\delta}(1)} .
$$

This follows from the LFPP distance exponent computation in [12]. To be more precise, [12, Theorem 1.5] shows that for continuum LFPP defined using the circle average process of the GFF, as in (1.5), the $\delta$-LFPP distance between the left and 
right boundaries of $\mathbb{S}$ is of order $\delta^{1-\xi Q+o_{\delta}(1)}$ with probability tending to 1 as $\delta \rightarrow 0$. Combining this with [12, Lemma 3.7] shows that the same is true for continuum LFPP defined using the white-noise approximation $\left\{\widehat{h}_{\delta}\right\}_{\delta>0}$, as defined in [12, Equation (3.1)], in place of the circle average process. The same argument as in the proof of [12, Proposition 3.16] then shows that (3.19) holds if we replace the circle average by the white-noise approximation in the definition of $\widetilde{D}_{h}^{\delta}$ (here we note that the definition of discretized LFPP in [12, Equation (3.32)] has an extra factor of $\delta$ as compared to (3.15), which is why we get $\delta^{-\xi Q+o_{\delta}(1)}$ instead of $\left.\delta^{1-\xi Q+o_{\delta}(1)}\right)$. The desired formula (3.19) now follows by combining this with the uniform comparison of $h_{\delta}$ and $\widehat{h}_{\delta}$ from [12, Lemma 3.7].

For the proof of Theorem 1.5 (and at several later places in this section) we will use the following terminology.

Definition 3.7 (Distance around an annulus) For a set $A \subset \mathbb{C}$ with the topology of a an annulus, we define the $D_{h}$-distance around $A$ to be the infimum of the $D_{h}$-lengths of the paths in $A$ which disconnect the inner and outer boundaries of $A$.

Proof of Theorem 1.5 Step 1: estimates for $D_{h}$. For $z \in \varepsilon \mathbb{Z}^{2}$, we write $S_{z}^{\varepsilon}$ for the square of side length $\varepsilon$ centered at $z$ and $B_{\varepsilon}\left(S_{z}^{\varepsilon}\right)$ for the $\varepsilon$-neighborhood of this square. Fix $\zeta \in(0,1)$. By Proposition 3.1 and a union bound over all $z \in(\mathbb{r} \mathbb{S}) \cap\left(\delta \mathrm{r} \mathbb{Z}^{2}\right)$, it holds with superpolynomially high probability as $\delta \rightarrow 0$ that (in the terminology of Definition 3.7)

$$
\left(D_{h} \text {-distance around } B_{\delta \mathfrak{r}}\left(S_{z}^{\delta \mathfrak{r}}\right) \backslash S_{z}^{\delta \mathfrak{r}}\right) \leq \delta^{-\zeta} \mathfrak{c}_{\delta \mathfrak{r}} e^{\xi h_{\delta \mathfrak{r}}}(z), \quad \forall z \in(\mathbb{r} \mathbb{S}) \cap\left(\delta \mathfrak{r} \mathbb{Z}^{2}\right) .
$$

Similarly, it holds with superpolynomially high probability as $\delta \rightarrow 0$ that

$$
D_{h}\left(S_{z}^{\delta \mathrm{r}}, \partial B_{\delta \mathrm{r}}\left(S_{z}^{\delta \mathrm{r}}\right)\right) \geq \delta^{\zeta} \mathfrak{c}_{\delta \mathrm{r}} e^{\xi h_{\delta \mathrm{r}}(z)}, \quad \forall z \in(\mathbb{r} \mathbb{S}) \cap\left(\delta \mathbb{r} \mathbb{Z}^{2}\right) .
$$

Henceforth assume that (3.20) and (3.21) both hold.

Step 2: lower bound for $\mathfrak{c}_{\delta \mathfrak{r}} / \mathfrak{c}_{\mathfrak{r}}$. Let $\pi:[0,|\pi|]_{\mathbb{Z}} \rightarrow(\mathrm{r} \mathbb{S}) \cap\left(\delta \mathrm{r} \mathbb{Z}^{2}\right)$ be a path in $(\mathrm{r} \mathbb{S}) \cap\left(\delta \mathrm{r} \mathbb{Z}^{2}\right)$ (with the graph structure defined by (3.14)) from $\partial_{\mathrm{L}}^{\delta \mathrm{r}}(\mathrm{r} \mathbb{S})$ to $\partial_{\mathrm{R}}^{\delta \mathrm{r}}(\mathrm{r} \mathbb{S})$ for which the sum in (3.15) equals $\widetilde{D}_{h}^{\delta \mathrm{r}}\left(\partial_{\mathrm{L}}^{\delta \mathrm{r}}(\mathrm{r} \mathbb{S}), \partial_{\mathrm{R}}^{\delta \mathrm{r}}(\mathrm{r} \mathbb{S}) ; \mathrm{r} \mathbb{S}\right)$. For each $j \in[0,|\pi|]_{\mathbb{Z}}$, let $P_{j}$ be a path in $B_{\delta \mathrm{r}}\left(S_{\pi(j)}^{\delta \mathrm{r}}\right) \backslash S_{\pi(j)}^{\delta \mathrm{r}}$ which disconnects the inner and outer boundaries of $B_{\delta \mathrm{r}}\left(S_{\pi(j)}^{\delta \mathrm{r}}\right) \backslash S_{\pi(j)}^{\delta \mathrm{r}}$ and whose $D_{h}$-length is at most $2 \delta^{-\zeta} \mathfrak{c}_{\delta \mathrm{r}} e^{\xi h_{\delta \mathrm{r}}}(z)$. Such a path exists by (3.20).

We have $P_{j} \cap P_{j-1} \neq \emptyset$ for each $j \in[0,|\pi|]_{\mathbb{Z}}$, so the union of the $P_{j}$ 's is connected and contains a path between the left and right boundaries of $r \mathbb{S}$. Therefore, the triangle inequality implies that

$$
\begin{aligned}
D_{h}\left(\mathrm{r} \partial_{\mathrm{L}} \mathbb{S}, \mathrm{r} \partial_{\mathrm{R}} \mathbb{S}\right) \leq \sum_{j=0}^{|\pi|}\left(D_{h} \text {-length of } P_{j}\right) & \leq 2 \delta^{-\zeta} \mathfrak{c}_{\delta \mathrm{r}} \sum_{j=0}^{|\pi|} e^{\xi h_{\delta \mathrm{r}}(0)} \\
& =2 \delta^{-\zeta} \mathfrak{c}_{\delta \mathrm{r}} \widetilde{D}_{h}^{\delta \mathrm{r}}\left(\partial_{\mathrm{L}}^{\delta \mathfrak{r}}(\mathbb{r} \mathbb{S}), \partial_{\mathrm{R}}^{\delta \mathfrak{r}}(\mathbb{r} \mathbb{S}) ; \mathfrak{r} \mathbb{S}\right) .
\end{aligned}
$$


By Axiom $\mathrm{V}$, the left side of (3.22) is at least $\delta^{\zeta} \mathfrak{c}_{\mathbb{T}} e^{\xi h_{\mathrm{r}}(0)}$ with probability tending to 1 as $\delta \rightarrow 0$, uniformly in r. By Lemma 3.6, the right side of (3.22) is at most $\delta^{-\xi Q-2 \zeta} \mathfrak{c}_{\delta \mathrm{r}} e^{\xi h_{\mathrm{r}}(0)}$ with probability tending to 1 as $\delta \rightarrow 0$, uniformly in $\mathrm{r}$. Combining these relations and sending $\zeta \rightarrow 0$ shows that $\mathfrak{c}_{\mathbb{r}} \leq \delta^{-\xi Q-o_{\delta}(1)} \mathfrak{c}_{\delta \mathrm{r}}$, as desired.

Step 3: upper bound for $\mathfrak{c}_{\delta \mathrm{r}} / \mathfrak{c}_{\mathbb{r}}$. Let $P:[0,|P|] \rightarrow \mathbb{S}$ be a path between the left and right boundaries of $r \mathbb{S}$ with $D_{h}$-length at most $2 D_{h}\left(\mathrm{r} \partial_{\mathrm{L}} \mathbb{S}, \mathrm{r} \partial_{\mathrm{R}} \mathbb{S} ; \mathrm{r} \mathbb{S}\right)$. We will use $P$ to construct a path in $(r \mathbb{S}) \cap\left(\delta \mathbb{R}^{2}\right)$ from $\partial_{\mathrm{L}}^{\delta \mathrm{r}}(\mathrm{r} \mathbb{S})$ to $\partial_{\mathrm{R}}^{\delta \mathrm{r}}(\mathrm{r} \mathbb{S})$ for which the sum in (3.15) can be bounded above.

To this end, let $\tau_{0}=0$ and let $z_{0} \in(\mathbb{r} \mathbb{S}) \cap\left(\delta \mathrm{r} \mathbb{Z}^{2}\right)$ be chosen so that $P(0) \in S_{z_{0}}^{\delta \mathrm{r}}$. Inductively, suppose $j \in \mathbb{N}$, a time $\tau_{j-1} \in[0,|P|]$, and a point $z_{j-1} \in(\mathbb{r} \mathbb{S}) \cap\left(\delta \mathbb{r} \mathbb{Z}^{2}\right)$ have been defined in such a way that $P\left(\tau_{j-1}\right) \in S_{z_{j-1}}^{\delta \mathrm{r}}$. Let $\tau_{j}$ be the first time after $\tau_{j-1}$ at which $P$ exits $B_{\delta \mathrm{r}}\left(S_{z_{j-1}}^{\delta \mathrm{r}}\right)$, if such a time exists, and otherwise set $\tau_{j}=|P|$. Let $z_{j} \in(\mathbb{r} \mathbb{S}) \cap\left(\delta \mathrm{r} \mathbb{Z}^{2}\right)$ be chosen so that $P\left(\tau_{j}\right) \in S_{z_{j}}^{\delta \mathrm{r}}$. Let $J$ be the smallest $j \in \mathbb{N}$ for which $\tau_{j}=|P|$, and note that $P(|P|) \in S_{z_{j}}^{\delta \mathrm{r}}$.

Successive squares $S_{z_{j-1}}^{\delta \mathrm{r}}$ and $S_{z_{j}}^{\delta \mathrm{r}}$ necessarily share a vertex. Hence $z_{j-1}$ and $z_{j}$ lie at $(\mathrm{r} \mathbb{S}) \cap\left(\delta \mathbb{r} \mathbb{Z}^{2}\right)$-graph distance 1 from one another, so $\pi(j):=z_{j}$ for $j \in[0, J]_{\mathbb{Z}}$ is a path from $\partial_{\mathrm{L}}^{\delta \mathrm{r}}(\mathrm{r} \mathbb{S})$ to $\partial_{\mathrm{R}}^{\delta \mathrm{r}}(\mathrm{r} \mathbb{S})$ in $(\mathrm{r} \mathbb{S}) \cap\left(\delta \mathrm{r} \mathbb{Z}^{2}\right)$.

We will now bound $\sum_{j=0}^{J} e^{\xi h_{\delta \mathrm{r}}}(\pi(j))$. For each $j \in[1, J]_{\mathbb{Z}}$, the path $P$ crosses between the inner and outer boundaries of $B_{\delta \mathrm{r}}\left(S_{z_{j-1}}^{\delta \mathrm{r}}\right) \backslash S_{z_{j-1}}^{\delta \mathrm{r}}$ between time $\tau_{j-1}$ and time $\tau_{j}$. By (3.21), for each $j \in[1, J]_{\mathbb{Z}}$,

$$
D_{h}\left(P\left(\tau_{j-1}\right), P\left(\tau_{j}\right)\right) \geq \delta^{\zeta} \mathfrak{c}_{\delta \mathrm{r}} e^{\xi h_{\delta \mathrm{r}}(\pi(j))} .
$$

Using (3.23) and the definition of $P$, we therefore have

$$
\begin{aligned}
\sum_{j=0}^{J} e^{\xi h_{\delta \mathrm{r}}(\pi(j))} & \leq \delta^{-\zeta} \mathfrak{c}_{\delta \mathbb{r}}^{-1} \sum_{j=0}^{J} D_{h}\left(P\left(\tau_{j-1}\right), P\left(\tau_{j}\right)\right) \\
& \leq \delta^{-\zeta} \mathfrak{c}_{\delta \mathbb{r}}^{-1} D_{h}\left(\mathbb{r} \partial_{\mathrm{L}} \mathbb{S}, \mathbb{r} \partial_{\mathrm{R}} \mathbb{S}\right)
\end{aligned}
$$

By Axiom V, the right side of (3.24) is at most $\delta^{-2 \zeta} \mathfrak{c}_{\delta \mathrm{r}}^{-1} \mathfrak{c}_{\mathrm{r}} e^{\xi h_{\mathrm{r}}(0)}$ with probability tending to 1 as $\delta \rightarrow 0$, uniformly in $\mathrm{r}$. By Lemma 3.6, the left side of (3.22) is at least $\delta^{-\xi Q-\zeta} e^{\xi h_{\mathrm{r}}(0)}$ with probability tending to 1 as $\delta \rightarrow 0$, uniformly in $\mathbb{r}$. Combining these relations and sending $\zeta \rightarrow 0$ shows that $\mathfrak{c}_{\delta \mathrm{r}}^{-1} \mathfrak{c}_{\mathrm{r}} \geq \delta^{-\xi Q-o_{\delta}(1)}$.

Theorem 1.5 has the following useful corollary.

Lemma 3.8 Let h be a whole-plane GFF normalized so that $h_{1}(0)=0$. Almost surely, for every compact set $K \subset \mathbb{C}$ we have $\lim _{r \rightarrow \infty} D_{h}\left(K, \partial B_{r}(0)\right)=\infty$. In particular, every closed, $D_{h}$-bounded subset of $\mathbb{C}$ is compact.

Proof By tightness across scales (Axiom V), there exists $a>0$ such that for each $r>0, \mathbb{P}\left[D_{h}\left(B_{r}(0), B_{2 r}(0)\right) \geq a \mathfrak{c}_{r} e^{\xi h_{r}(0)}\right] \geq 1 / 2$. By the locality of $D_{h}$ (Axiom II) and since $\sigma\left(\left.\bigcap_{r>0} h\right|_{\mathbb{C} \backslash B_{r}(0)}\right)$ is trivial, a.s. there are infinitely many $k \in \mathbb{N}$ for which $D_{h}\left(B_{2^{k}}(0), B_{2^{k+1}}(0)\right) \geq a \mathfrak{c}_{2^{k}} e^{\xi h_{2^{k}}(0)}$. By Theorem 1.5, $\mathfrak{c}_{r}=r^{\xi Q+o_{r}(1)}$. Since 
$t \mapsto h_{e^{t}}(0)$ is a standard linear Brownian motion [16, Section 3.1], we get that a.s. $\lim _{r \rightarrow \infty} \mathfrak{c}_{r} e^{\xi h_{r}(0)}=\infty$. Hence a.s. $\lim _{\sup _{k \rightarrow \infty}} D_{h}\left(B_{2^{k}}(0), B_{2^{k+1}}(0)\right)=\infty$. Since $D_{h}$ is a length metric, for any $r \geq 2^{k+1}$ and any compact set $K \subset B_{2^{k}}(0)$, we have $D_{h}\left(K, \partial B_{r}(0)\right) \geq D_{h}\left(B_{2^{k}}(0), B_{2^{k+1}}(0)\right)$. We thus obtain the first assertion of the lemma. The first assertion (applied with $K$ equal to a single point, say) implies that any $D_{h}$-bounded subset of $\mathbb{C}$ must be contained in a Euclidean-bounded subset of $\mathbb{C}$, which must be compact since $D_{h}$ induces the Euclidean topology on $\mathbb{C}$.

\subsection{Moment bound for diameters}

In this section we will prove the following more quantitative version of the moment bound from Theorem 1.8, which is required to be uniform across scales.

Proposition 3.9 Let $U \subset \mathbb{C}$ be open and let $K \subset U$ be a compact connected set with more than one point. For each $p \in\left(-\infty, 4 d_{\gamma} / \gamma^{2}\right)$, there exists $C_{p}>0$ which depends on $U$ and $K$ but not on $\mathbb{r}$ such that for each $\mathbb{r}>0$,

$$
\mathbb{E}\left[\left(\mathfrak{c}_{\mathrm{r}}^{-1} e^{-\xi h_{\mathrm{r}}(0)} \sup _{z, w \in \mathrm{r} K} D_{h}(z, w ; \mathbb{r} U)\right)^{p}\right] \leq C_{p} .
$$

We will deduce Proposition 3.9 from the following variant, which allows us to bound internal $D_{h}$-distances all the way up to the boundary of a square. Recall that $\mathbb{S}:=(0,1)^{2}$.

Proposition 3.10 For each $p \in\left(-\infty, 4 d_{\gamma} / \gamma^{2}\right)$, there is a constant $C_{p}>0$ such that for each $\mathrm{r}>0$,

$$
\mathbb{E}\left[\left(\mathfrak{c}_{\mathbb{r}}^{-1} e^{-\xi h_{\mathrm{r}}(0)} \sup _{z, w \in \mathbb{R} \mathbb{S}} D_{h}(z, w ; \mathbb{r} \mathbb{S})\right)^{p}\right] \leq C_{p} .
$$

Proof of Proposition 3.9, assuming Proposition 3.10

For $p<0$, the bound (3.25) follows from the lower bound of Proposition 3.1. Now assume $p \in\left(0,4 d_{\gamma} / \gamma^{2}\right)$. We can cover $K$ by finitely many Euclidean squares $S_{1}, \ldots, S_{n}$ which are contained in $U$, chosen in a manner depending only on $K$ and $U$. For $k=1, \ldots, n$, let $u_{k}$ be the bottom left corner of $S_{k}$ and let $\rho_{k}$ be its side length. Proposition 3.10 together with Axiom IV shows that there is a constant $\widetilde{C}_{p}>0$ depending only on $p$ such that for each $k=1, \ldots, n$,

$$
\mathbb{E}\left[\left(\mathfrak{c}_{\mathbb{r} \rho_{k}}^{-1} e^{-\xi h_{\mathbb{r} \rho_{k}}\left(\mathfrak{r} u_{k}\right)} \sup _{z, w \in \mathbb{r} S_{k}} D_{h}\left(z, w ; \mathbb{r} S_{k}\right)\right)^{p}\right] \leq \widetilde{C}_{p} .
$$

We apply the Gaussian tail bound to bound each of the Gaussian random variables $h_{\mathbb{r} \rho_{k}}\left(\mathrm{r} u_{k}\right)-h_{\mathbb{r}}(0)$ (which have constant order variance) and Theorem 1.5 to compare $\mathfrak{c}_{\mathbb{r} \rho_{k}}$ to $\mathfrak{c}_{\mathbb{r}}$ up to a constant-order multiplicative error. This allows us to deduce (3.25) from (3.27). 
To prove Proposition 3.10, we first use the upper bound in Proposition 3.1 and a union bound to build paths between the two shorter sides of each $2^{-n} \mathbb{r} \times 2^{-n-1}$ r or $2^{-n-1} \mathbb{r} \times 2^{-n}$ r rectangle with corners in $2^{-n-1} \mathbb{r} \mathbb{Z}^{2}$ which is contained in $\mathbb{S}$. We then string together such paths at all scales (in the manner illustrated in Fig. 3) to get a bound for the internal $D_{h}$-diameter of $r \mathbb{S}$. The following lemma is needed to control the circle average terms which appear when we apply Proposition 3.1.

Lemma 3.11 Fix $R>0$ and $q>2$. For $C>1$ and $\mathbb{r}>0$, it holds with probability $1-C^{-q-\sqrt{q^{2}-4}+o_{C}(1)}$ as $C \rightarrow \infty$, at a rate which is uniform in $\mathbb{r}$, that

$$
\sup \left\{\left|h_{2^{-n} \mathbb{r}}(w)-h_{\mathbb{r}}(0)\right|: w \in B_{R \mathbb{r}}(0) \cap\left(2^{-n-1} \mathbb{r} \mathbb{Z}^{2}\right)\right\} \leq \log \left(C 2^{q n}\right), \quad \forall n \in \mathbb{N}_{0}
$$

When we apply Lemma 3.11, we will take $q$ to be a little bit less than $Q=$ $2 / \gamma+\gamma / 2$. The fact that $Q+\sqrt{Q^{2}-4}=4 / \gamma$ is the reason why $\gamma$ (instead of just $\xi)$ appears in our moment bounds.

Proof of Lemma 3.11 To lighten notation, define the event

$$
E_{\mathbb{r}}^{n}:=\left\{\sup \left\{\left|h_{2^{-n_{\mathbb{r}}}}(w)-h_{\mathbb{r}}(0)\right|: w \in B_{R \mathbb{r}}(0) \cap\left(2^{-n-1} \mathbb{r} \mathbb{Z}^{2}\right)\right\} \leq \log \left(C 2^{q n}\right)\right\} .
$$

We want a lower bound for the probability that $E_{\mathrm{r}}^{n}$ occurs for every $n \in \mathbb{N}_{0}$ simultaneously.

Fix $\zeta>0$ (which we will eventually send to 0 ) and a partition $\zeta=\alpha_{0}<\cdots<$ $\alpha_{N}=1 / \zeta$ of $[\zeta, 1 / \zeta]$ with $\max _{k=1, \ldots, N}\left(\alpha_{k}-\alpha_{k-1}\right) \leq \zeta$. We will separately bound the probability of $E_{\mathbb{r}}^{n}$ for $2^{n} \in\left[C^{\alpha_{k-1}}, C^{\alpha_{k}}\right]$ for $k=1, \ldots, N$, for $2^{n} \geq C^{1 / \zeta}$, and for $2^{n} \leq C^{\zeta}$.

By Lemma 3.4 applied with $\varepsilon=2^{-n}, v=0$, and $q+1 / \alpha_{k}$ in place of $q$, we find that for each $k=1, \ldots, N$ and each $n \in \mathbb{N}_{0}$ with $2^{n} \in\left[C^{\alpha_{k-1}}, C^{\alpha_{k}}\right]$,

$$
\begin{aligned}
\mathbb{P}\left[\left(E_{\mathbb{r}}^{n}\right)^{c}\right] & \leq \mathbb{P}\left[\sup \left\{\left|h_{2^{-n_{\mathbb{r}}}}(w)-h_{\mathbb{r}}(0)\right|: w \in B_{R \mathbb{r}}(0) \cap\left(2^{-n-1} \mathbb{r} \mathbb{Z}^{2}\right)\right\}\right. \\
& \left.>\left(q+\frac{1}{\alpha_{k}}\right) \log \left(2^{n}\right)\right] \\
& \leq 2^{-n\left(\frac{\left(q+1 / \alpha_{k}\right)^{2}}{2}-2\right)} \leq C^{-\alpha_{k-1}\left(\frac{\left(q+1 / \alpha_{k}\right)^{2}}{2}-2\right)} \leq C^{2 \alpha_{k}-\frac{\left(q \alpha_{k}+1\right)^{2}}{2 \alpha_{k}}+o_{\zeta}(1)}
\end{aligned}
$$

with the rate of the $o_{\zeta}(1)$ depending only on $q$. Note that in the last inequality, we have done some trivial algebraic manipulations then used that $\alpha_{k}-\alpha_{k-1} \leq \zeta$ (which is what produces the $o_{\zeta}(1)$ ). By a union bound over logarithmically many (in $C$ ) values 
of $n \in \mathbb{N}_{0}$ with $2^{n} \in\left[C^{\alpha_{k-1}}, C^{\alpha_{k}}\right]$, we get

$$
\mathbb{P}\left[E_{\mathbb{r}}^{n}, \forall n \in \mathbb{N}_{0} \text { with } C^{\alpha_{k-1}} \leq 2^{n} \leq C^{\alpha_{k}}\right] \geq 1-C^{2 \alpha_{k}-\frac{\left(q \alpha_{k}+1\right)^{2}}{2 \alpha_{k}}+o_{\zeta}(1)+o_{C}(1)} .
$$

For $n \in \mathbb{N}_{0}$ with $2^{n} \geq C^{1 / \zeta}$, Lemma 3.4 applied with $\varepsilon=2^{-n}, v=0$, and $q+\zeta$ in place of $q$ gives

$$
\mathbb{P}\left[\left(E_{\mathrm{r}}^{n}\right)^{c}\right] \leq 2^{-n\left((q+\zeta)^{2} / 2-2\right)} .
$$

Summing this estimate over all such $n$ shows that

$$
\mathbb{P}\left[E_{\mathrm{r}}^{n}, \forall n \in \mathbb{N} \text { with } 2^{n} \geq C^{1 / \zeta}\right] \geq 1-C^{-\frac{(q+\zeta)^{2}-4}{2 \zeta}+o_{C}(1)}
$$

Finally, if $n \in \mathbb{N}_{0}$ and $2^{n} \leq C^{\zeta}$, then the Gaussian tail bound and a union bound, applied as in the proof of Lemma 3.4, shows that $\mathbb{P}\left[\left(E_{\mathrm{r}}^{n}\right)^{c}\right] \leq C^{2 \zeta-(q \zeta+1)^{2} /(2 \zeta)+o_{C}(1)}$ (in fact, if $2^{n}$ is of constant order, this probability will decay superpolynomially in $C$ due to the Gaussian tail bound). By a union bound over a logarithmic number (in $C$ ) of such values of $n$ we get

$$
\mathbb{P}\left[E_{\mathrm{r}}^{n}, \forall n \in \mathbb{N} \text { with } 2^{n} \leq C^{\zeta}\right] \geq 1-C^{2 \zeta-\frac{(q \zeta+1)^{2}}{2 \zeta}+o_{C}(1)}
$$

The quantity $2 \alpha-(q \alpha+1)^{2} /(2 \alpha)$ is maximized over all $\alpha>0$ when $\alpha=\left(q^{2}-\right.$ $4)^{-1 / 2}$, in which case it equals $-\left(q+\sqrt{q^{2}-4}\right)$. Consequently, by combining the estimates (3.31), (3.32), and (3.33), we get that if $\zeta$ is chosen sufficiently small relative to $q$, then

$$
\mathbb{P}\left[E_{\mathbb{r}}^{n}, \forall n \in \mathbb{N}_{0}\right] \geq 1-C^{-q-\sqrt{q^{2}-4}+o_{\zeta}(1)+o_{C}(1)} .
$$

Sending $\zeta \rightarrow 0$ now concludes the proof.

Proof of Proposition 3.10 For $p<0$, the bound (3.26) follows from the lower bound of Proposition 3.1. We will bound the positive moments up to order $4 d_{\gamma} / \gamma^{2}$.

Step 1: Constructing short paths across rectangles. Fix $q \in(2, Q)$ which we will eventually send to $Q$. By Lemma 3.11 it holds with probability $1-C^{-q-\sqrt{q^{2}-4}+o_{C}(1)}$ that

$$
\sup \left\{\left|h_{2^{-n_{\mathbb{r}}}}(w)-h_{\mathbb{r}}(0)\right|: w \in \mathbb{r} \mathbb{S} \cap\left(2^{-n-1} \mathbb{r} \mathbb{Z}^{2}\right)\right\} \leq \log \left(C 2^{q n}\right), \quad \forall n \in \mathbb{N}_{0} .
$$

Now fix $\zeta \in(0, Q-q)$, which we will eventually send to zero. For $n \in \mathbb{N}_{0}$, let $\mathcal{R}_{\mathbb{r}}^{n}$ be the set of open $2^{-n} \mathbb{r} \times 2^{-n-1} \mathbb{r}$ or $2^{-n-1} \mathbb{r} \times 2^{-n}$ r rectangles $R \subset \mathbb{r} \mathbb{S}$ with corners in $2^{-n-1} \mathbb{r} \mathbb{Z}^{2}$. For $R \in \mathcal{R}_{\mathbb{r}}^{n}$ let $w_{R}$ be the bottom-left corner of $R$. 
Fig. 3 Three of the sets $X_{S_{n}(z)}$ for dyadic squares containing $z$ used in the proof of

Proposition 3.10. As $n \rightarrow \infty$, the $D_{h}$-diameter of $S_{n}(z)$

shrinks to zero (by the continuity of $\left.(z, w) \mapsto D_{h}(z, w)\right)$, so the distance from $z$ to $X_{S_{N}(z)}$ is bounded above by the sum over all $n \geq N$ of the $D_{h}$-lengths of the four paths which comprise $X_{S_{n}(z)}$

Let

\section{et}

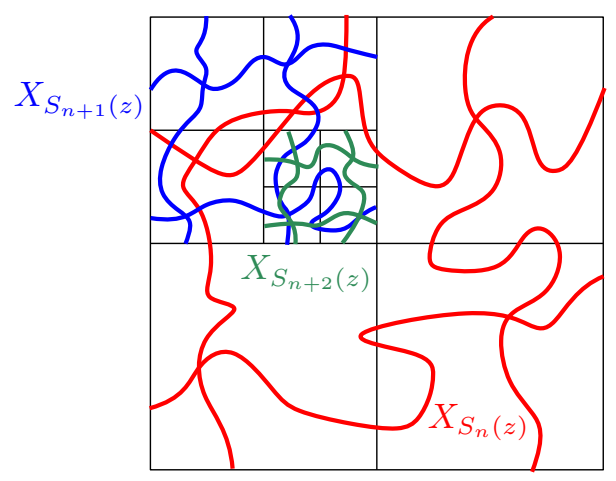

$$
N_{C}:=\left\lfloor\log _{2} C^{\zeta}\right\rfloor
$$

By the upper bound of Proposition 3.1 (applied with $2^{-n_{\mathbb{r}}}$ in place of $\mathbb{r}$ and with $A=2^{\zeta \xi n}$ ), Axiom IV, and a union bound over all $R \in \mathcal{R}_{\mathrm{r}}^{n}$ and all $n \geq N_{C}$, we get that except on an event of probability decaying faster than any negative power of $C$ (the rate of decay depends on $\zeta$ ), the following is true. For each $n \geq N_{C}$ and each $R \in \mathcal{R}_{\mathrm{r}}^{n}$, the distance between the two shorter sides of $R$ w.r.t. the internal metric $D_{h}(\cdot, \cdot ; R)$ is at most $2^{\zeta \xi n} \mathfrak{c}_{2-n_{\mathrm{r}}} e^{\xi h_{2-n_{\mathbb{T}}}\left(w_{R}\right)}$.

Combining this with (3.10) shows that with probability $1-C^{-q-\sqrt{q^{2}-4}+o_{C}(1)}$, it holds for each $n \geq N_{C}$ and each $R \in \mathcal{R}_{\mathbb{r}}^{n}$ that there is a path $P_{R}$ in $R$ between the two shorter sides of $R$ with $D_{h}$-length at most $C^{\xi} 2^{(q+\zeta) \xi n} c_{2^{-n}} e^{\xi h_{\mathrm{r}}(0)}$. By applying Theorem 1.5 to bound $\mathfrak{c}_{2}{ }^{-n_{r}}$, we get that in fact

$$
\left(D_{h} \text {-length of } P_{R}\right) \leq C^{\xi} 2^{-(Q-q-\zeta) \xi n+o_{n}(n)} \mathfrak{c}_{\mathbb{r}} e^{\xi h_{\mathbb{r}}(0)}
$$

Henceforth assume that such paths $P_{R}$ exist. We will establish an upper bound for the $D_{h}$-diameter of $\mathrm{r} \mathbb{S}$.

Step 2: stringing together paths in rectangles. For each square $S \subset \mathbb{r} S$ with side length $2^{-n} \mathbb{r}$ and corners in $2^{-n} \mathbb{r} \mathbb{S}$, there are exactly four rectangles in $\mathcal{R}_{\mathbb{r}}^{n}$ which are contained in $S$. If $n \geq N_{C}$, let $X_{S}$ be the \#-shaped region which is the union of the paths $P_{R}$ for these four rectangles, as illustrated in Fig. 3. If $S^{\prime}$ is one of the four dyadic children of $S$, then $X_{S} \cap X_{S^{\prime}} \neq \emptyset$. Since the four paths which comprise $X_{S}$ have $D_{h}$-length at most $C^{\xi} 2^{-(Q-q-\zeta) \xi n+o_{n}(n)} e^{\xi h_{\mathrm{r}}(0)} \mathfrak{c}_{\mathbb{r}} e^{\xi h_{\mathrm{r}}(0)}$, this means that each point of $X_{S}$ can be joined to $X_{S^{\prime}}$ by a path in $S$ of $D_{h}$-length at most $C^{\xi} 2^{-(Q-q-\zeta) \xi n+o_{n}(n)} \mathfrak{c}_{\mathrm{r}} e^{\xi h_{\mathbb{1}}(0)}$.

Since the metric $D_{h}$ is a continuous function on $\mathbb{C} \times \mathbb{C}$, if $z \in \mathbb{r} \mathbb{S}$ and we let $S_{n}(z)$ for $n \in \mathbb{N}_{0}$ be the square of side length $2^{-n} \mathbb{r}$ with corners in $2^{-n} \mathbb{r} \mathbb{Z}^{2}$ which contains $z$, so that $S_{0}(z)=\mathbb{S}$, then the $D_{h}$-diameter of $S_{n}(z)$ tends to zero as $n \rightarrow \infty$. 
Consequently,

$$
\sup _{w \in S_{N_{C}}(z)} D_{h}(z, w ; \mathbb{r} \mathbb{S}) \leq C^{\xi} \mathfrak{c}_{\mathbb{r}} e^{\xi h_{\mathbb{r}}(0)} \sum_{n=N_{C}}^{\infty} 2^{-(Q-q-\zeta) \xi n+o_{n}(n)} \leq O_{C}\left(C^{\xi}\right) \mathfrak{c}_{\mathbb{r}} e^{\xi h_{\mathbb{r}}(0)}
$$

Since this holds for every $z \in \mathbb{r} \mathbb{S}$, we get that with probability at least 1 $C^{-q-\sqrt{q^{2}-4} o_{C}(1)}$, for each $n \geq N_{C}$, each $2^{-n_{\mathbb{r}}} \times 2^{-n_{\mathbb{r}}}$ square $S \subset \mathbb{r} \mathbb{S}$ with corners in $2^{-n} \mathbb{r} \mathbb{Z}^{2}$ has $D_{h}(\cdot, \cdot ; \mathrm{r} \mathbb{S})$-diameter at most $O_{C}\left(C^{\xi}\right) \mathfrak{c}_{\mathbb{r}} e^{\xi h_{\mathrm{r}}(0)}$.

Step 3: conclusion. Since $2^{N_{C}} \leq C^{\zeta}$, we can use the triangle inequality to get that if the event at the end of the preceding step occurs, then the $D_{h}(\cdot, \cdot ; \mathrm{r} \mathbb{S})$-diameter of $\mathbb{r} \mathbb{S}$ is at most $O_{C}\left(C^{\xi+\zeta}\right) \mathfrak{c}_{\mathbb{r}} e^{\xi h_{\mathbb{r}}(0)}$. Setting $\widetilde{C}:=C^{\xi+\zeta}$, then sending $\zeta \rightarrow 0$, shows that

$$
\mathbb{P}\left[\mathfrak{c}_{\mathrm{r}}^{-1} e^{-\xi h_{\mathrm{r}}(0)} \sup _{z, w \in \mathbb{r} \mathbb{S}} D_{h}(z, w ; \mathbb{r} \mathbb{S})>\widetilde{C}\right] \leq \widetilde{C}^{-\xi^{-1}\left(q+\sqrt{q^{2}-4}\right)+o} \widetilde{C}^{(1)} .
$$

By sending $q \rightarrow Q$ and noting that $Q+\sqrt{Q^{2}-4}=4 / \gamma$, we get

$$
\mathbb{P}\left[\mathfrak{c}_{\mathbb{r}}^{-1} e^{-\xi h_{\mathrm{r}}(0)} \sup _{z, w \in \mathbb{r} \mathbb{S}} D_{h}(z, w ; \mathbb{r} \mathbb{S})>\widetilde{C}\right] \leq \widetilde{C}^{-\frac{4}{\gamma \xi}+\sigma^{(1)}}=\widetilde{C}^{-\frac{4 d_{\gamma}}{\gamma^{2}}+o^{(1)}} .
$$

For $p \in\left(0,4 d_{\gamma} / \gamma^{2}\right)$, we can multiply this last estimate by $\widetilde{C}^{p-1}$ and integrate to get the desired $p$ th moment bound (3.26).

\subsection{Pointwise distance bounds}

In this subsection we will prove the following more quantitative versions of Theorems 1.10 and 1.11, which are required to be uniform across scales. Recall that $h$ is a whole-plane GFF normalized so that $h_{1}(0)=0$.

Proposition 3.12 (Distance from a point to a circle) Let $\alpha \in \mathbb{R}$ and let $h^{\alpha}:=h-$ $\alpha \log |\cdot|$. If $\alpha \in(-\infty, Q)$, then for each $p \in\left(-\infty, \frac{2 d_{\gamma}}{\gamma}(Q-\alpha)\right)$, there exists $C_{p}>0$ such that for each $\mathrm{r}>0$,

$$
\mathbb{E}\left[\left(\mathfrak{c}_{\mathbb{T}}^{-1} \mathbb{r}^{\alpha \xi} e^{-\xi h_{\mathbb{r}}(0)} D_{h^{\alpha}}\left(0, \partial B_{\mathbb{r}}(0)\right)\right)^{p}\right] \leq C_{p}
$$

If $\alpha>Q$, then a.s. $D_{h^{\alpha}}(0, z)=\infty$ for every $z \in \mathbb{C} \backslash\{0\}$.

Proposition 3.13 (Distance between two points) Let $\alpha, \beta \in \mathbb{R}$, let $z, w \in \mathbb{C}$ be distinct, and let $h^{\alpha, \beta}:=h-\alpha \log |\cdot-z|-\beta \log |\cdot-w|$. Set $\mathbb{r}:=|z-w| / 2$. If $\alpha, \beta \in(-\infty, Q)$, then for each $p \in\left(-\infty, \frac{2 d_{\gamma}}{\gamma}(Q-\max \{\alpha, \beta\})\right)$, there exists $C_{p}>0$ such that for each choice of $z, w$ as above,

$$
\mathbb{E}\left[\left(\mathfrak{c}_{\mathrm{r}}^{-1} \mathrm{r}^{\alpha \xi} e^{-\xi h_{\mathrm{r}}(z)} D_{h^{\alpha}}\left(z, w ; B_{8 \mathrm{r}}(z)\right)\right)^{p}\right] \leq C_{p}
$$


If either $\alpha>Q$ or $\beta>Q$, then a.s. $D_{h^{\alpha, \beta}}(z, w)=\infty$.

Propositions 3.12 and 3.13 are immediate consequences of the following sharper distance estimates and a calculation for the standard linear Brownian motion $t \mapsto$ $h_{\mathrm{r} e^{-t}}(0)-h_{\mathrm{r}}(0)$.

Proposition 3.14 Assume that we are in the setting of Proposition 3.12. If $\alpha \in$ $(-\infty, Q)$, then there is a deterministic function $\psi:[0, \infty) \rightarrow[0, \infty)$ which is bounded in every neighborhood of 0 and satisfies $\lim _{t \rightarrow \infty} \psi(t) / t=0$, depending only on $\alpha$ and the choice of metric $D,{ }^{6}$ such that the following is true. For each $\mathbb{r}>0$, it holds with superpolynomially high probability as $C \rightarrow \infty$, at a rate which is uniform in the choice of $\mathbb{r}$, that

$$
\begin{aligned}
& C^{-1} \frac{\mathfrak{c}_{\mathbb{r}}}{\mathbb{r}^{\alpha \xi}} \int_{0}^{\infty} e^{\xi h_{\mathbb{r} e^{-t}}(0)-\xi(Q-\alpha) t-\psi(t)} d t \leq D_{h^{\alpha}}\left(0, \partial B_{\mathbb{r}}(0)\right) \\
& \leq C \frac{\mathfrak{c}_{\mathbb{r}}}{\mathbb{r}^{\alpha \xi}} \int_{0}^{\infty} e^{\xi h_{\mathbb{r} e^{-t}}(0)-\xi(Q-\alpha) t+\psi(t)} d t
\end{aligned}
$$

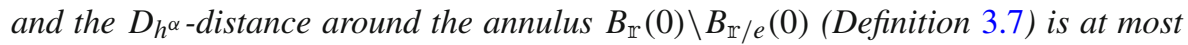
the right side of (3.40). If $\alpha>Q$, then a.s. $D_{h^{\alpha}}(0, z)=\infty$ for every $z \in \mathbb{C} \backslash\{0\}$.

Proposition 3.15 Assume that we are in the setting of Proposition 3.13. If $\alpha, \beta \in$ $(-\infty, Q)$, then there is a deterministic function $\psi:[0, \infty) \rightarrow[0, \infty)$ which is bounded in every neighborhood of 0 and satisfies $\lim _{t \rightarrow \infty} \psi(t) / t=0$, depending only on $\alpha$ and the choice of metric $D$, such that the following is true. With superpolynomially high probability as $C \rightarrow \infty$, at a rate which is uniform in the choice of $z$ and $w$,

$$
D_{h^{\alpha, \beta}}(z, w) \geq C^{-1} \frac{\mathfrak{c}_{\mathbb{r}}}{\mathbb{r}^{\alpha \xi}} \int_{0}^{\infty}\left(e^{\xi h_{\mathbb{r} e}-t(z)-\xi(Q-\alpha) t-\psi(t)}+e^{\xi h_{\mathbb{r} e^{-t}}(w)-\xi(Q-\beta) t-\psi(t)}\right) d t
$$

and

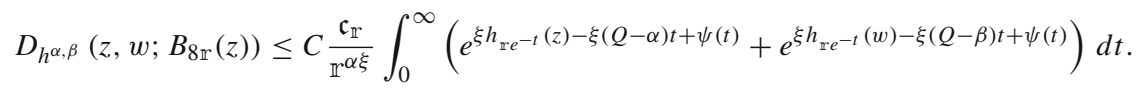

If either $\alpha>Q$ or $\beta>Q$, then a.s. $D_{h^{\alpha, \beta}}(z, w)=\infty$.

Remark 3.16 It will be shown in [25] that every weak LQG metric is a strong LQG metric, so in particular it satisfies Axiom $\mathrm{V}$ with $\mathfrak{c}_{r}=r^{\xi Q}$. Once this is established, our proof shows that Propositions 3.14 and 3.15 hold with $\psi(t)=0$.

\footnotetext{
6 At this point we do not know that the weak LQG metric $D: h \mapsto D_{h}$ is unique (it will be proven that this metric is unique up to a deterministic multiplicative constant in [25]). When we say that something is allowed to depend on the choice of $D$, we mean that it is allowed to depend on which particular weak LQG metric we are looking at.
} 
Proof of Proposition 3.12, assuming Proposition 3.14

For $t \geq 0$, let $B_{t}:=h_{\mathrm{r} e^{-t}}(0)-h_{\mathrm{r}}(0)$. Then $B$ is a standard linear Brownian motion [16, Section 3.1]. By Proposition 3.14, for each $\zeta \in(0,1)$, it holds with superpolynomially high probability as $C \rightarrow \infty$, uniformly over the choice of $\mathrm{r}$, that

$$
\begin{aligned}
& C^{-\zeta} \int_{0}^{\infty} e^{\xi B_{t}-(Q-\alpha) \xi t-\zeta t} d t \leq \mathfrak{c}_{\mathrm{r}}^{-1} \mathbb{r}^{\alpha \xi} e^{-\xi h_{\mathbb{T}}(0)} D_{h^{\alpha}}\left(0, \partial B_{\mathbb{r}}(0)\right) \\
& \leq C^{\zeta} \int_{0}^{\infty} e^{\xi B_{t}-(Q-\alpha) \xi t+\zeta t} d t
\end{aligned}
$$

To prove the proposition, we will use an exact formula for the laws of the integrals appearing in (3.43). To write down such a formula, let $\widetilde{B}_{s}:=\xi B_{s / \xi^{2}}$. Then $\widetilde{B}$ is a standard linear Brownian motion and $B_{t}=\xi^{-1} \widetilde{B}_{\xi^{2} t}$. Making the change of variables $t=s / \xi^{2}$ gives

$$
\int_{0}^{\infty} e^{\xi B_{t}-(Q-\alpha) \xi t+\zeta t} d t=\frac{1}{\xi^{2}} \int_{0}^{\infty} e^{\widetilde{B}_{s}-(Q-\alpha) s / \xi+\zeta s / \xi^{2}} d s
$$

It is shown in [17] (see also [48, Example 3.3] with $\left.c=(Q-\alpha) / \xi-\zeta / \xi^{2}\right)$ that

$$
\mathbb{P}\left[\int_{0}^{\infty} e^{\widetilde{B}_{s}-(Q-\alpha) s / \xi+\zeta s / \xi^{2}} d s \in d x\right]=b x^{-2(Q-\alpha) / \xi+2 \zeta / \xi^{2}-1} e^{-2 / x}, \quad \forall x \geq 0,
$$

where $b$ is a normalizing constant depending only on $Q, \alpha, \xi$. Combining the upper bound in (3.43) with (3.44) and the upper tail asymptotics of the density (3.45), then sending $\zeta \rightarrow 0$, shows that

$$
\mathbb{P}\left[\mathfrak{c}_{\mathbb{r}}^{-1} \mathbb{r}^{\alpha \xi} e^{-\xi h_{\mathbb{r}}(0)} D_{h^{\alpha}}\left(0, \partial B_{\mathbb{r}}(0)\right)>C\right] \leq C^{-2(Q-\alpha) / \xi-o_{C}(1)},
$$

uniformly in $\mathrm{r}$. Recall that $\xi=\gamma / d_{\gamma}$. Multiplying both sides of (3.46) by $p C^{p-1}$ and integrating gives the desired bound for positive moments from (3.38). We similarly obtain the desired bound for negative moments using the lower bound in (3.43) and the exponential lower tail of the density (3.45).

\section{Proof of Proposition 3.13, assuming Proposition 3.15}

The bound for positive moments in (3.39) is obtained in essentially the same way as the analogous bound in Proposition 3.12. We apply the upper bound in Proposition 3.15 and use the exact formula (3.45) to bound the integral of each of the two summands appearing on the right side of (3.42), then multiply the resulting tail estimate by $p C^{p-1}$ and integrate. We use that $h_{\mathbb{r}}(z)-h_{\mathbb{r}}(w)$ is Gaussian with constant-order variance to get an estimate which depends only on $h_{\mathrm{r}}(z)$, not $h_{\mathrm{r}}(w)$. The bound for negative moments in (3.39) can similarly be extracted from the lower bound in Proposition 3.15, or can be deduced from Proposition 3.12 and the fact that a path from $z$ to $w$ must cross $\partial B_{\mathbb{I}}(z)$. 


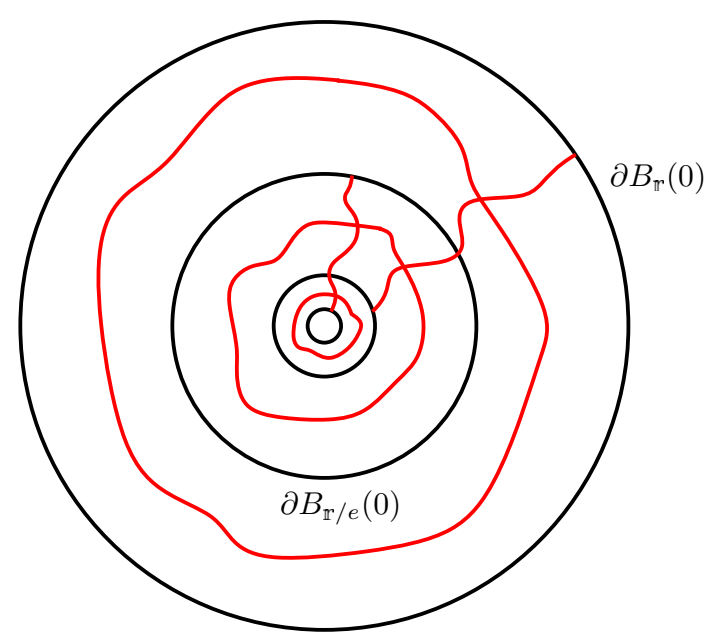

Fig. 4 To prove Proposition 3.14, we use Proposition 3.1 to show that with high probability, the following bounds hold simultaneously for each $k \in \mathbb{N}_{0}$ : a lower bound for the $D_{h}$-distance across the annulus $B_{\mathrm{r} e^{-k}}(0) \backslash B_{\mathrm{rr} e^{-k-1}}(0)$; an upper bound for the $D_{h}$-distance around this annulus; and a lower bound for the $D_{h}$-distance across the larger annulus $B_{\mathrm{r} e^{-k}}(0) \backslash B_{\mathrm{r} e^{-k-2}}(0)$. Summing the lower bounds for the distances across these annuli leads to the lower bound in (3.40). The paths involved in the upper bounds are shown in red in the figure. Concatenating all of these paths gives a path from 0 to $\partial B_{\mathbb{I}}(0)$, which leads to the upper bound in (3.40) (color figure online)

It remains only to prove Propositions 3.14 and 3.15. We will prove Proposition 3.14 by applying Proposition 3.1 to bound the distances across and around concentric annuli surrounding 0 with dyadic radii, then summing over all of these annuli (see Fig. 4 for an illustration). We will then deduce Proposition 3.15 from Proposition 3.14 by considering two overlapping Euclidean disks centered at $z$ and $w$, respectively. For this purpose the statement concerning the $D_{h}$-distance around $B_{\mathbb{I}}(0) \backslash B_{\mathbb{I} / e}(0)$ is essential to link up paths in these two disks.

Proof of Proposition 3.14 See Fig. 2 for an illustration. The proof is divided into four steps.

1. We apply Proposition 3.1 in the annuli $\mathbb{A}_{\mathbb{r} e^{-k-1}, \mathbb{r} e^{-k}}$ for $k \in \mathbb{N}_{0}$ to prove upper and lower bounds for $D_{h}\left(0, \partial B_{\mathrm{r}}(0)\right)$ in terms of sums over such annuli.

2. Using Brownian motion estimates, we convert from sums over annuli to integrals of quantities of the form $e^{\xi h_{\mathrm{re}}-t(z)-\xi(Q-\alpha) t+o_{t}(t)}$.

3. We show that the contribution of the small error terms in our estimates coming from sums/integrals at superpolynomially small scales is negligible.

4. We put the above pieces together to conclude the proof.

Step 1: applying Proposition 3.1 at exponential scales. We will apply Proposition 3.1 and take a union bound over exponential scales. In this step we allow any value of $\alpha \in \mathbb{R}$.

Fix a small parameter $\zeta \in(0,1)$, which we will eventually send to zero. By Proposition 3.1 and Axiom III (to deal with the addition of $-\alpha \log |\cdot|$ ) and a union bound over 
all $k \in\left[0, C^{1 / \zeta}\right]_{\mathbb{Z}}$, we find that with superpolynomially high probability as $C \rightarrow \infty$, the following is true for each $k \in\left[0, C^{1 / \zeta}\right]_{\mathbb{Z}}$.

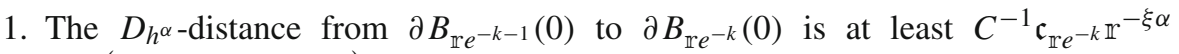
$\exp \left(\xi h_{\mathbb{r} e^{-k}}(0)+\xi \alpha k\right)$.

2. There is a path from $\partial B_{\mathbb{r} e^{-k-2}}(0)$ to $\partial B_{\mathbb{r} e^{-k}}(0)$ which has $D_{h^{\alpha}}$-length at most $C \mathfrak{c}_{\mathfrak{r} e^{-k}} \mathbb{r}^{-\xi \alpha} \exp \left(\xi h_{\mathfrak{r} e^{-k}}(0)+\xi \alpha k\right)$. Moreover, there is also a path in $B_{\mathbb{r} e^{-k}}(0) \backslash \overline{B_{\mathbb{r} e^{-k-1}}(0)}$ which disconnects $\partial B_{\mathbb{r} e^{-k-1}}(0)$ from $\partial B_{\mathbb{r} e^{-k}}(0)$ and which has $D_{h^{\alpha}}$-length at most

$C \mathfrak{c}_{\mathbb{r} e^{-k} \mathbb{I}^{-\xi \alpha}} \exp \left(\xi h_{\mathbb{r} e^{-k}}(0)+\xi \alpha k\right)$.

To deal with the scales for which $k \geq C^{1 / \zeta}$, we apply Proposition 3.1 with $k^{\zeta}$ in place of $C$ and take a union bound over all such values of $k$ to find that superpolynomially high probability as $C \rightarrow \infty$, the above two conditions hold for each $k \in\left[0, C^{1 / \zeta}\right]_{\mathbb{Z}}$, and furthermore the following condition holds for each integer $k \geq C^{1 / \zeta}$.

$2^{\prime}$. There is a path from $\partial B_{\mathrm{r} e^{-k-2}}(0)$ to $\partial B_{\mathrm{r} e^{-k}}(0)$ which has $D_{h^{\alpha}}$-length at most $k^{\zeta} \mathfrak{c}_{\mathrm{r} e^{-k}} \mathbb{r}^{-\xi \alpha} \exp \left(\xi h_{\mathrm{r} e^{-k}}(0)+\xi \alpha k\right)$. Moreover, there is also a path in $B_{\text {r } e^{-k}}(0) \backslash \overline{B_{\text {re } e^{-k-1}}(0)}$ which disconnects $\partial B_{\text {r } e^{-k-1}}(0)$ from $\partial B_{\mathbb{r} e^{-k}}(0)$ and which has $D_{h^{\alpha}}$-length at most

$k^{\zeta} \mathfrak{c}_{\mathbb{r} e^{-k}} \mathbb{1}^{-\xi \alpha} \exp \left(\xi h_{\mathrm{r} e^{-k}}(0)+\xi \alpha k\right)$.

Henceforth assume that conditions 1 and 2 hold for each $k \in\left[0, C^{1 / \zeta}\right]_{\mathbb{Z}}$ and condition $2^{\prime}$ holds for each integer $k \geq C^{1 / \zeta}$, which happens with superpolynomially high probability as $C \rightarrow \infty$.

Any path from 0 to $\partial B_{\mathbb{r}}(0)$ must cross each of the annuli $B_{\mathbb{r} e^{-k}}(0) \backslash B_{\mathrm{r} e^{-k-1}}(0)$ for $k \in\left[0, C^{1 / \zeta}\right]_{\mathbb{Z}}$. Furthermore, the union of $\{0\}$ and the paths from conditions 2 and $2^{\prime}$ for all $k \in \mathbb{N}_{0}$ contains a path from 0 to $\partial B_{\mathrm{r}}(0)$. By Theorem 1.5 , there is a deterministic function $\phi:[0, \infty) \rightarrow[0, \infty)$ with $\phi(k)=o_{k}(k)$, depending only on the choice of metric $D$, such that

$$
e^{-\xi Q k-\phi(k)} \mathfrak{c}_{\mathbb{r}} \leq \mathfrak{c}_{\mathbb{r} e^{-k}} \leq e^{-\xi Q k+\phi(k)} \mathfrak{c}_{\mathbb{r}}, \quad \forall \mathbb{r}>0
$$

Summing the bounds from conditions 1 and 2 over all $k \in\left[0, C^{1 / \zeta}\right]_{\mathbb{Z}}$ and the bounds from condition $2^{\prime}$ over all integers $k \geq C^{1 / \zeta}$ and plugging in (3.47) shows that with superpolynomially high probability as $C \rightarrow \infty$,

$$
\begin{aligned}
& C^{-1} \frac{\mathfrak{c}_{\mathbb{r}}}{\mathbb{r}^{\alpha \xi}} \sum_{k=0}^{\left\lfloor C^{1 / \zeta}\right\rfloor} e^{\xi h_{\mathrm{r} e^{-k}}(0)-\xi(Q-\alpha) k-\phi(k)} \leq D_{h^{\alpha}}\left(0, \partial B_{\mathbb{r}}(0)\right) \\
& \quad \leq C \frac{\mathfrak{c}_{\mathbb{r}}}{\mathfrak{r}^{\alpha \xi}} \sum_{k=0}^{\left\lfloor C^{1 / \zeta}\right\rfloor} e^{\xi h_{\mathrm{r} e^{-k}}(0)-\xi(Q-\alpha) k+\phi(k)}+\frac{\mathfrak{c}_{\mathbb{r}}}{\mathbb{r}^{\alpha \xi}} \sum_{k=\left\lfloor C^{1 / \zeta}\right\rfloor+1}^{\infty} k^{\zeta} e^{\xi h_{\mathbb{r} e^{-k}}(0)-\xi(Q-\alpha) k+\phi(k)} .
\end{aligned}
$$

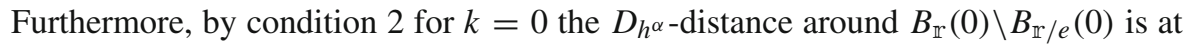
most the right side of (3.48). 
Step 2: From summation to integration. We now want to convert from sums to integrals in (3.48). Since $t \mapsto h_{\mathbb{r} e^{-t}}(0)-h_{\mathbb{r}}(0)$ is a standard linear Brownian motion [16, Section 3.1], the Gaussian tail bound and the union bound show that with superpolynomially high probability as $C \rightarrow \infty$,

$$
\sup _{t \in[k, k+1]}\left|h_{\mathbb{r} e^{-t}}(0)-h_{\mathbb{r} e^{-k}}(0)\right| \leq \frac{1}{\xi} \log C, \quad \forall k \in\left[0, C^{1 / \zeta}\right]_{\mathbb{Z}} .
$$

Let $\psi(t):=\phi(\lfloor t\rfloor)$, where $\phi$ is as in (3.47). Then $\psi(t)=o_{t}(t)$ and if (3.49) holds, then for each $k \in\left[0, C^{1 / \zeta}\right]_{\mathbb{Z}}$,

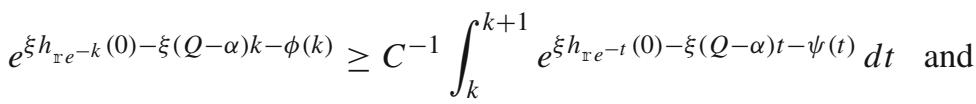

$$
\begin{aligned}
& e^{\xi h_{\mathrm{r} e^{-k}}(0)-\xi(Q-\alpha) k+\phi(k)} \leq C \int_{k}^{k+1} e^{\xi h_{\mathrm{r} e^{-t}}(0)-\xi(Q-\alpha) t+\psi(t)} d t .
\end{aligned}
$$

By summing (3.50) over all $k \in\left[0, C^{1 / \zeta}\right]_{\mathbb{Z}}$, we obtain

$$
\begin{aligned}
& \sum_{k=0}^{\left\lfloor C^{1 / \zeta}\right\rfloor} e^{\xi h_{\mathbb{r} e^{-k}}(0)-\xi(Q-\alpha) k-\phi(k)} \geq C^{-1} \int_{0}^{\left\lfloor C^{1 / \zeta}\right\rfloor+1} e^{\xi h_{\mathbb{r} e^{-t}(0)-\xi(Q-\alpha) t-\psi(t)}} d t \text { and } \\
& \sum_{k=0}^{\left\lfloor C^{1 / \zeta}\right\rfloor} e^{\xi h_{\mathbb{r} e^{-k}}(0)-\xi(Q-\alpha) k+\phi(k)} \leq C \int_{0}^{\left\lfloor C^{1 / \zeta}\right\rfloor+1} e^{\xi h_{\mathbb{r} e^{-t}}(0)-\xi(Q-\alpha) t+\psi(t)} d t
\end{aligned}
$$

Step 3: Bounding the sum of the small scales. To deduce our desired bounds from (3.48) and (3.51), we now need an upper bound for $\int_{\left.\mid C^{1 / \zeta}\right\rfloor}^{\infty} e^{\xi h_{\mathrm{r} e}-t(0)-\xi(Q-\alpha) t+\psi(t)} d t$ and an upper bound for the second sum on the right side of (3.48). This is the only step where we need to assume that $\alpha<Q$.

Since $t \mapsto h_{\mathbb{r} e^{-t}}(0)-h_{\mathrm{r}}(0)$ is a standard linear Brownian motion and for $q \in(0,1]$, $x \mapsto x^{q}$ is concave, hence subadditive, if $q \in(0,1]$ is chosen small enough that $\xi q(Q-\alpha)-\xi^{2} q^{2} / 2>0$, then

$$
\begin{aligned}
\mathbb{E} & {\left[\left(\int_{\left\lfloor C^{1 / \zeta}\right\rfloor}^{\infty} e^{\xi h_{\mathrm{r} e^{-t}}(0)-\xi(Q-\alpha) t+\psi(t)} d t\right)^{q}\right] } \\
& \preceq e^{q h_{\mathrm{r}}(0)} \int_{\left\lfloor C^{1 / \zeta}\right\rfloor}^{\infty} \exp \left(-\left(\xi q(Q-\alpha)-\frac{\xi^{2} q^{2}}{2}\right) t+o_{t}(t)\right) d t \\
& \preceq e^{q h_{\mathbb{T}}(0)} \exp \left(-\frac{1}{2}\left(\xi q(Q-\alpha)-\frac{\xi^{2} q^{2}}{2}\right) C^{1 / \zeta}\right)
\end{aligned}
$$

where here the $o_{t}(t)$ and the implicit constants in $\preceq$ do not depend on $C$ or $\mathbb{r}$. Therefore, the Chebyshev inequality shows that

$$
\mathbb{P}\left[\int_{\left\lfloor C^{1 / \zeta}\right\rfloor}^{\infty} e^{\xi h_{\mathrm{r} e}-t(0)-\xi(Q-\alpha) t+\psi(t)} d t>e^{\xi h_{\mathbb{I}}(0)-C^{1 /(2 \zeta)}}\right]
$$


decays faster than any negative power of $C$. On the other hand, it is easily seen from the Gaussian tail bound that

$$
\mathbb{P}\left[\int_{0}^{\left\lfloor C^{1 / \zeta}\right\rfloor} e^{\xi h_{\mathbb{r} e^{-t}}(0)-\xi(Q-\alpha) t+\psi(t)} d t<e^{\xi h_{\mathbb{r}}(0)-C^{1 /(2 \zeta)}}\right]
$$

decays faster than any negative power of $C$. Hence with superpolynomially high probability as $C \rightarrow \infty$,

$$
\int_{0}^{\infty} e^{\xi h_{\text {re } e^{-t}}(0)-\xi(Q-\alpha) t+\psi(t)} d t \leq 2 \int_{0}^{\left\lfloor C^{1 / \zeta}\right\rfloor} e^{\xi h_{\mathrm{r} e}-t(0)-\xi(Q-\alpha) t+\psi(t)} d t .
$$

Similarly, we get that with superpolynomially high probability as $C \rightarrow \infty$,

$$
\sum_{k=\left\lfloor C^{1 / \zeta}\right\rfloor+1}^{\infty} k^{\zeta} e^{\xi h_{\mathrm{r} e^{-k}}(0)-\xi(Q-\alpha) k-\phi(k)} \leq \int_{0}^{\infty} e^{\xi h_{\mathrm{r} e}-t(0)-\xi(Q-\alpha) t-\psi(t)} d t .
$$

Step 4: Conclusion. By applying (3.51), (3.54), and (3.55) to bound the left and right sides of (3.48), we get that if $\alpha<Q$, then with superpolynomially high probability, the bounds (3.40) as well as the bound stated just below (3.40) [here we use the sentence just below (3.48)] all hold with $2 C^{2}$, say, in place of $C$. Since we are claiming that these bounds hold with superpolynomially high probability as $C \rightarrow \infty$, this is sufficient.

Finally, we consider the case when $\alpha>Q$. Since $h_{\mathrm{r} e^{-t}(0)-h_{\mathrm{r}}(0) \text { evolves as }}$ a standard linear Brownian motion, for each $\beta \in(0, \alpha-Q)$ it is a.s. the case that the summand $e^{\xi h_{r e}-k(0)-\xi(Q-\alpha) k-\phi(k)}$ in the lower bound in (3.48) is bounded below by $e^{\beta k}$ for large enough $k$. (How large is random). Since (3.48) holds with superpolynomially high probability as $C \rightarrow \infty$, the Borel-Cantelli lemma combined with the preceding sentence shows that a.s. for large enough (random) $C>1$, we have $D_{h^{\alpha}}\left(0, \partial B_{\mathbb{I}}(0)\right) \geq C^{-1} e^{\beta\left\lfloor C^{1 / \zeta}\right\rfloor}$, which tends to $\infty$ as $C \rightarrow \infty$. This shows that a.s. $D_{h^{\alpha}}\left(0, \partial B_{\mathbb{r}}(0)\right)=\infty$. Since this holds a.s. for each rational $\mathbb{r}>0$, it follows that a.s. $D_{h^{\alpha}}(0, z)=\infty$ for every $z \in \mathbb{C} \backslash\{0\}$.

Proof of Proposition 3.15 We first observe that by Axiom IV, Proposition 3.14 still holds with 0 replaced by any $z \in \mathbb{C}$, with the rate of convergence as $C \rightarrow \infty$ uniform in $z$ and $\mathbb{r}$. Applying the lower bound of Proposition 3.14 with each of $z$ and $w$ in place of 0 immediately gives (3.41) since any path from $z$ to $w$ must contain disjoint sub-paths from $z$ to $\partial B_{\mathbb{r} / 2}(z)$ and from $w$ to $B_{\mathbb{r} / 2}(w)$. Moreover, by comparing the local behavior of $D_{h^{\alpha, \beta}}$ near $z$ and near $w$ to $D_{h^{\alpha}}$ and $D_{h^{\beta}}$, respectively, we get that a.s. $D_{h^{\alpha, \beta}}(z, w)=\infty$ if either $\alpha>Q$ or $\beta>Q$.

It remains to prove (3.42). Assume $\alpha<Q$. We first apply Proposition 3.14 with 8 r in place of $\mathrm{r}$ to find that with superpolynomially high probability as $C \rightarrow \infty$, there is a path $P_{z, 1}$ from $z$ to $\partial B_{8 \mathrm{r}}(z)$ and a path $P_{z, 2}$ in $B_{\mathbb{r}}(z) \backslash B_{8 \mathrm{r} / e}(z)$ which disconnects $\partial B_{8 \mathrm{r} / e}(z)$ from $\partial B_{8 \mathrm{r}}(z)$ which each have $D_{h}$-length at most

$$
\int_{-\log 8}^{\infty} e^{\xi h_{\mathrm{r}} e^{-t}(z)-\xi(Q-\alpha) t+\psi(t)} d t
$$


and the same is true with $w$ in place of $z$. Since $w \in B_{8 \mathrm{r} / e}(z)$, the union of the paths $P_{z, 1}, P_{z, 2}$, and $P_{w, 1}$ contains a path from $z$ to $w$ in $B_{8 \mathrm{r}}(z)$. This gives (3.42) but with $-\log 8$ instead of 0 in the lower bound of integration for the integral on the right.

To get the estimate with the desired lower bound of integration, we use that $t \mapsto h_{\mathbb{r} e^{-t}}(z)-h_{\mathbb{r}}(z)$ is a standard two-sided linear Brownian motion. In particular, two applications of the Gaussian tail bound show that with superpolynomially high probability as $C \rightarrow \infty$,

$$
\sup _{t \in[-\log 8,0]} h_{\mathrm{r} e^{-t}}(z) \leq \inf _{t \in[0, \log 2]} h_{\mathbb{r} e^{-t}}(z)+\log C .
$$

Therefore, with superpolynomially high probability as $C \rightarrow \infty$,

$$
\begin{aligned}
\int_{-\log 8}^{\infty} e^{\xi h_{\mathbb{r} e^{-t}}(z)-\xi(Q-\alpha) t+\psi(t)} d t \leq & \int_{0}^{\infty} e^{\xi h_{\mathrm{r} e^{-t}}(z)-\xi(Q-\alpha) t+\psi(t)} d t \\
& +C^{\xi} \int_{0}^{\log 2} e^{\xi h_{\mathrm{r} e^{-t}}(z)-\xi(Q-\alpha) t+\psi(t)} d t .
\end{aligned}
$$

Combining this with the analogous estimate with $w$ in place of $z$ and the aforementioned analog of (3.42) with $-\log 8$ instead of 0 in the lower bound of integration gives (3.42).

Although it is not needed for the proofs of Propositions 3.14 and 3.15, we record the following generalization of Proposition 3.9 which tells us in particular that $D_{h^{\alpha}}$ induces the Euclidean topology on $\mathbb{C}$ when $Q>2$ and $\alpha<Q$ (which is a stronger statement than just that $D_{h^{\alpha}}(0, z)<\infty$ for every $\left.z \in \mathbb{C}\right)$.

Proposition 3.17 Let $h, \alpha, h^{\alpha}$, and $D_{h^{\alpha}}$ be as in Proposition 3.14. If $Q=2 / \gamma+\gamma / 2>$ 2 and $\alpha \in(-\infty, Q)$, then for each $-\infty<p<\min \left\{\frac{4 d_{\gamma}}{\gamma^{2}}, \frac{2 d_{\gamma}}{\gamma}(Q-\alpha)\right\}$, there exists $C_{\alpha, p}>0$ such that for each $\mathbb{r}>0$,

$$
\mathbb{E}\left[\left(e^{-\xi h_{\mathbb{r}}(0)} \mathfrak{c}_{\mathbb{r}}^{-1} \mathbb{r}^{\alpha \xi} \sup _{z, w \in B_{\mathbb{r}}(0)} D_{h^{\alpha}}(z, w)\right)^{p}\right] \leq C_{\alpha, p}
$$

In particular, a.s. $D_{h^{\alpha}}$ induces the Euclidean topology on $\mathbb{C}$.

We note that the range of moments $-\infty<p<\min \left\{\frac{4 d_{\gamma}}{\gamma^{2}}, \frac{2 d_{\gamma}}{\gamma}(Q-\alpha)\right\}$ for the $D_{h^{\alpha}}$-diameter of $\mathbb{D}$ appearing in Proposition 3.17 is the same as the range of moments

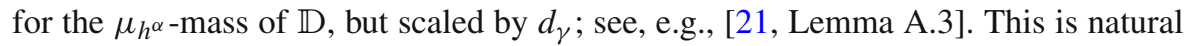
from the perspective that $d_{\gamma}$ is the scaling exponent relating $\gamma$-LQG distances and areas.

Proof of Proposition 3.17 On $B_{\mathbb{r}}(0) \backslash B_{\mathbb{r} / 2}(0)$, we have that $-\alpha \log |\cdot|$ is bounded above and below by $-\alpha \log$ r times constants depending only on $\alpha$. Therefore, the existence of negative moments is immediate from Axiom III and Proposition 3.9 applied with $U=\mathbb{D} \backslash B_{1 / 2}(0)$. 
To get the desired positive moments, for $k \in \mathbb{N}_{0}$ let $A_{k}$ be the annulus $B_{\mathbb{r} e^{-k}}(0) \backslash B_{\mathbb{r} e^{-k-1}}(0)$. The random variable $h_{\mathbb{r} e^{-k}}(0)-h_{\mathbb{r}}(0)$ is Gaussian with variance $k$, so for $p>0$,

$$
\mathbb{E}\left[e^{p \xi\left(h_{r e}-k(0)-h_{\mathbb{r}}(0)\right)}\right]=e^{p^{2} \xi^{2} k / 2}, \quad \forall p>0
$$

By Proposition 3.9 (applied with $K=A_{0}, U=\mathbb{C}$, and $r e^{-k}$ in place of $r$ ),

$$
\mathbb{E}\left[\left(\mathfrak{c}_{\mathbb{r} e^{-k}}^{-1} e^{-\xi h_{\mathrm{r} e^{-k}}(0)} e^{-\alpha \xi k_{\mathbb{r}}^{\alpha k}} \sup _{z, w \in A_{k}} D_{h^{\alpha}}(z, w)\right)^{p}\right] \preceq 1, \quad \forall p<\frac{4 d_{\gamma}}{\gamma^{2}}
$$

By (3.57) and (3.58) and since $\left.\left(h-h_{\mathbb{r} e^{-k}}(0)\right)\right|_{A_{k}}$ is independent from $h_{\mathrm{r} e^{-k}}(0)-h_{\mathbb{r}}(0)$, we find that for $p \in\left(0,4 d_{\gamma} / \gamma^{2}\right)$,

$$
\begin{aligned}
\mathbb{E} & {\left[\left(e^{-\xi h_{\mathbb{T}}(0)} \mathfrak{c}_{\mathbb{r}}^{-1} \mathbb{r}^{\alpha \xi} \sup _{z, w \in A_{k}} D_{h^{\alpha}}(z, w)\right)^{p}\right] } \\
= & \left(\frac{\mathfrak{c}_{\mathbb{r} e^{-k}}}{\mathfrak{c}_{\mathbb{T}}}\right)^{p} e^{p \alpha \xi k} \mathbb{E}\left[e^{p \xi\left(h_{\mathbb{r} e^{-k}}(0)-h_{\mathbb{r}}(0)\right)}\right] \\
& E\left[\left(\mathfrak{c}_{\mathbb{r} e^{-k}}^{-1} e^{-\xi h_{\mathbb{r} e^{-k}}(0)} e^{-\alpha \xi k_{\mathbb{r}}^{\alpha k}} \sup _{z, w \in A_{k}} D_{h^{\alpha}}(z, w)\right)^{p}\right] \\
\leq & \exp \left(-\left(\xi p(Q-\alpha)-\frac{p^{2} \xi^{2}}{2}\right) k+o_{k}(k)\right),
\end{aligned}
$$

at a rate depending only on $\alpha, p$. Note that in the last line we used Theorem 1.5 to bound $\mathfrak{c}_{\mathbb{r} e^{-k}} / \mathfrak{c}_{\mathbb{r}}$.

The quantity inside the exponential on the right side of (3.59) is negative provided $p<\min \left\{\frac{4 d_{\gamma}}{\gamma^{2}}, \frac{2 d_{\gamma}}{\gamma}(Q-\alpha)\right\}$ (recall that $\left.\xi=\gamma / d_{\gamma}\right)$. For $0<p<\min \left\{1, \frac{2 d_{\gamma}}{\gamma}(Q-\alpha)\right\}$, the function $x \mapsto x^{p}$ is concave, hence subadditive, so summing (3.59) over all $k \in \mathbb{N}_{0}$ gives

$$
\begin{aligned}
& \mathbb{E}\left[\left(e^{-\xi h_{\mathbb{r}}(0)} \mathfrak{c}_{\mathbb{r}}^{-1} \mathbb{r}^{\alpha \xi} \sup _{z, w \in B_{\mathbb{r}}(0)} D_{h^{\alpha}}(z, w)\right)^{p}\right] \\
& \leq \sum_{k=0}^{\infty} \mathbb{E}\left[\left(e^{-\xi h_{\mathbb{r}}(0)} \mathfrak{c}_{\mathbb{r}}^{-1} \mathbb{r}^{\alpha \xi} \sup _{z, w \in A_{k}} D_{h^{\alpha}}(z, w)\right)^{p}\right] \\
& \preceq \sum_{k=0}^{\infty} \exp \left(-\left(\xi p(Q-\alpha)-\frac{p^{2} \xi^{2}}{2}\right) k+o_{k}(k)\right) \\
& \preceq 1 .
\end{aligned}
$$


This gives (3.56) in the case when $0<p<\min \left\{1, \frac{2 d_{\gamma}}{\gamma}(Q-\alpha)\right\}$. In the case when $1 \leq p<\min \left\{\frac{4 d_{\gamma}}{\gamma^{2}}, \frac{2 d_{\gamma}}{\gamma}(Q-\alpha)\right\}$, (3.56) follows from a similar calculation with the triangle inequality for the $L^{p}$ norm used in place of sub-additivity.

Finally, we know that the restriction of $D_{h^{\alpha}}$ to $\mathbb{C} \backslash\{0\}$ induces the Euclidean topology (see the discussion just above Theorem 1.10), so to check that that $D_{h^{\alpha}}$ induces the Euclidean topology, we need to show that a.s. $\sup _{z, w \in B_{e^{-k}}(0)} D_{h^{\alpha}}(z, w) \rightarrow 0$ as $k \rightarrow$ $\infty$. This follows from the bound (3.60) applied with $\mathbb{r}=1$ and the Borel-Cantelli lemma.

\subsection{Hölder continuity}

We will prove the following more quantitative version of Theorem 1.7 which is required to be uniform across scales.

Proposition 3.18 Fix a compact set $K \subset \mathbb{C}$ and exponents $\chi \in(0, \xi(Q-2))$ and $\chi^{\prime}>\xi(Q+2)$. For each $\mathbb{r}>0$, it holds with polynomially high probability as $\varepsilon \rightarrow 0$, at a rate which is uniform in $\mathbb{r}$, that

$$
\left|\frac{u-v}{\mathbb{r}}\right|^{\chi^{\prime}} \leq \mathfrak{c}_{\mathbb{r}}^{-1} e^{-\xi h_{\mathbb{r}}(0)} D_{h}(u, v) \leq\left|\frac{u-v}{\mathbb{r}}\right|^{\chi}, \quad \forall u, v \in \mathbb{r} K \text { with }|u-v| \leq \varepsilon \mathbb{r} .
$$

We will actually prove a slightly stronger version of the upper bound for $D_{h}$ in Proposition 3.18, which bounds internal distances relative to a small neighborhood of $u$ instead of just distances along paths in all of $\mathbb{C}$; see Lemma 3.20 just below. This stronger version is used in [25].

For the proof of Proposition 3.18, we assume that $Q>2$ and we fix a compact set $K \subset \mathbb{C}$. The basic idea of the proof of the upper bound in (3.61) is to apply Proposition 3.9 to Euclidean balls of radius $\varepsilon$ and take a union bound over many such Euclidean balls which cover $K$. The basic idea for the proof of the lower bound in (3.61) is to apply the lower bound in Proposition 3.1 to lower bound the $D_{h}$-distance across Euclidean annuli of the form $B_{2 \varepsilon}(z) \backslash B_{\varepsilon}(z)$, then take a union bound over many such annuli whose inner balls cover $K$. We first prove an upper bound for $D_{h}$-distances in terms of Euclidean distances. For this purpose we will use the following consequence of Propositions 3.9 and 3.10.

Lemma 3.19 For each $s \in(0, \xi Q)$, each $\mathbb{r}>0$, and each $z \in \mathbb{r} K$,

$$
\mathbb{P}\left[\sup _{u, v \in B_{\varepsilon \mathbb{r}}(z)} D_{h}\left(u, v ; B_{2 \varepsilon \mathbb{r}}(z)\right) \leq \varepsilon^{s} \mathfrak{c}_{\mathbb{r}} e^{\xi h_{\mathbb{r}}(0)}\right] \geq 1-\varepsilon^{\frac{(\xi Q-s)^{2}}{2 \xi^{2}}+o_{\varepsilon}(1)}, \quad \text { as } \varepsilon \rightarrow 0 \text {, }
$$

uniformly over the choices of $\mathbb{r}$ and $z \in \mathbb{r} K$. Furthermore, if we let $S^{\varepsilon \mathrm{r}}(z)$ be the square of side length $\varepsilon \mathbb{r}$ centered at $z$, then for $\mathbb{r}>0$ and $z \in \mathbb{r} K$, the $D_{h}$-internal 
diameter of $S^{\varepsilon \mathfrak{r}}(z)$ satisfies

$\mathbb{P}\left[\sup _{u, v \in S^{\varepsilon \mathrm{r}}(z)} D_{h}\left(u, v ; S^{\varepsilon \mathrm{r}}(z)\right) \leq \varepsilon^{s} \mathfrak{c}_{\mathrm{r}} e^{\xi h_{\mathrm{r}}(0)}\right] \geq 1-\varepsilon^{\frac{(\xi Q-s)^{2}}{2 \xi^{2}}+o_{\varepsilon}(1)}, \quad$ as $\varepsilon \rightarrow 0$,

uniformly over the choices of $\mathbb{1}$ and $z \in \mathbb{r} K$.

Proof We know that $h_{2 \varepsilon \mathrm{r}}(z)-h_{\mathrm{r}}(z)$ is centered Gaussian of variance $\log \varepsilon^{-1}-\log 2$ and is independent from $\left.\left(h-h_{2 \varepsilon \mathrm{r}}(z)\right)\right|_{B_{2 \varepsilon \mathrm{r}}(z)}$. By Axioms II and III, $h_{2 \varepsilon \mathrm{r}}(z)-h_{\mathrm{r}}(z)$ is also independent from the internal metric

$$
D_{h-h_{2 \varepsilon \mathbb{I}}(z)}\left(u, v ; B_{2 \varepsilon \mathbb{I}}(z)\right)=e^{-\xi h_{2 \varepsilon \mathbb{I}}(z)} D_{h}\left(u, v ; B_{2 \varepsilon \mathbb{I}}(z)\right) .
$$

Consequently, we can apply Theorem 1.5 and Proposition 3.9 (with $\varepsilon$ r in place of $\mathbb{r}$ ) together with the formula $\mathbb{E}\left[e^{X}\right]=e^{\operatorname{Var}(X) / 2}$ for a Gaussian random variable $X$ to get that for $p \in(0,4 /(\gamma \xi))$,

$$
\begin{aligned}
& \mathbb{E}\left[\left(\mathfrak{c}_{\mathrm{r}}^{-1} e^{-\xi h_{\mathrm{r}}(0)} \sup _{u, v \in B_{\varepsilon \mathrm{r}}(z)} D_{h}\left(u, v ; B_{2 \varepsilon \mathbb{\mathrm { r }}}(z)\right)\right)^{p}\right] \\
& =\left(\frac{\mathfrak{c}_{\varepsilon \mathrm{r}}}{\mathfrak{c}_{\mathrm{r}}}\right)^{p} \mathbb{E}\left[e^{\xi p\left(h_{\varepsilon \mathrm{r}}(z)-h_{\mathrm{r}}(z)\right.}\right] \mathbb{E}\left[\left(\mathfrak{c}_{\varepsilon \mathrm{r}}^{-1} e^{-\xi h_{\varepsilon \mathrm{r}}(z)} \sup _{u, v \in B_{\varepsilon \mathrm{r}}(z)} D_{h}\left(u, v ; B_{2 \varepsilon \mathbb{\mathrm { r }}}(z)\right)\right)^{p}\right] \\
& \leq \varepsilon^{\xi Q p-\xi^{2} p^{2} / 2+o_{\varepsilon}(1)},
\end{aligned}
$$

with the $o_{\varepsilon}(1)$ uniform over all $\mathbb{r}>0$ and $z \in \mathbb{C}$.

By (3.64) and the Chebyshev inequality,

$$
\mathbb{P}\left[\sup _{u, v \in B_{\varepsilon \mathbb{I}}(z)} D_{h}\left(u, v ; B_{2 \varepsilon \mathbb{r}}(z)\right)>\varepsilon^{s} \mathfrak{c}_{\mathbb{r}} e^{\xi h_{\mathbb{r}}(z)}\right] \leq \varepsilon^{p \xi Q-\frac{p^{2} \xi^{2}}{2}-p s+o_{\varepsilon}(1)}
$$

The exponent on the right side is maximized for $p=(\xi Q-s) / \xi^{2}$, which is always at most $4 /(\xi \gamma)$ for $s>0$ (since $\gamma<2$ ) and is positive provided $s<\xi Q$. Making this choice of $p$ gives (3.62) but with $h_{\mathbb{r}}(z)$ in place of $h_{\mathbb{r}}(0)$. The random variables $h_{\mathbb{r}}(z)-h_{\mathbb{r}}(0)$ for $z \in \mathbb{r} K$ are Gaussian with variance bounded above by a constant depending only on $K$. Consequently, we can apply the Gaussian tail bound to get (3.62) in general.

The bound (3.63) is proven similarly but with Proposition 3.10 used in place of Proposition 3.9.

We can now prove a slightly sharper version of the upper bound of Proposition 3.18. 
Lemma 3.20 For each $\chi \in(0, \xi(Q-2))$ and each $\mathbb{r}>0$, it holds with polynomially high probability as $\varepsilon \rightarrow 0$, at a rate which is uniform in $\mathbb{r}$, that

$$
\mathfrak{c}_{\mathrm{r}}^{-1} e^{-\xi h_{\mathbb{r}}(0)} D_{h}\left(u, v ; B_{2|u-v|}(u)\right) \leq\left|\frac{u-v}{\mathbb{r}}\right|^{\chi}, \quad \forall u, v \in \mathbb{r} K \text { with }|u-v| \leq \varepsilon \mathbb{r} .
$$

Furthermore, it also holds with polynomially high probability as $\varepsilon \rightarrow 0$, at a rate which is uniform in $\mathbb{r}$, that for each $k \in \mathbb{N}_{0}$ and each $2^{-k} \varepsilon \mathbb{r} \times 2^{-k} \varepsilon \mathbb{r}$ square $S$ with corners in $2^{-k} \varepsilon r \mathbb{Z}^{2}$ which intersects $\mathrm{r} K$, we have

$$
\mathfrak{c}_{\mathbb{r}}^{-1} e^{-\xi h_{\mathbb{r}}(0)} \sup _{u, v \in S} D_{h}(u, v ; S) \leq\left(2^{-k} \varepsilon\right)^{\chi} .
$$

Proof The bound (3.66) follows from (3.62), applied with $s=\chi$ and with $2^{-k} \varepsilon$ for $k \in \mathbb{N}_{0}$ in place of $\varepsilon$, together with a union bound over all $z \in B_{\varepsilon \mathrm{r}}(K) \cap\left(2^{-k-2} \varepsilon \mathbb{r} \mathbb{Z}^{2}\right)$ and then over all $k \in \mathbb{N}_{0}$. The bound (3.67) similarly follows from (3.63).

To prove the Hölder continuity of the Euclidean metric w.r.t. $D_{h}$, we first need the following estimate which plays a role analogous to Lemma 3.19.

Lemma 3.21 For each $s>\xi Q$, each $\mathbb{r}>0$, and each $z \in \mathbb{r} K$,

$$
\mathbb{P}\left[D_{h}\left(B_{\varepsilon \mathrm{r}}(z), \partial B_{2 \varepsilon \mathrm{r}}(z)\right) \geq \varepsilon^{s} \mathfrak{c}_{\mathbb{r}} e^{\xi h_{\mathrm{r}}(0)}\right] \geq 1-\varepsilon^{\frac{(s-\xi Q)^{2}}{2 \xi^{2}}+o_{\varepsilon}(1)}, \quad \text { as } \varepsilon \rightarrow 0,
$$

uniformly over the choices of $\mathbb{r}$ and $z \in \mathbb{r} K$.

Proof The proof is similar to that of Lemma 3.19 but we use Proposition 3.1 instead of Proposition 3.9. Proposition 3.1 implies that $\mathfrak{c}_{\varepsilon \mathrm{r}}^{-1} e^{-\xi h_{\varepsilon \mathrm{r}}(z)} D_{h}\left(B_{\varepsilon \mathrm{r}}(z), \partial B_{2 \varepsilon \mathrm{r}}(z)\right)$ has finite moments of all negative orders which are bounded above uniformly over all $z \in \mathbb{C}$ and $\mathbb{r}>0$. By the same calculation as in (3.64), for each $p>0$ we have

$$
\mathbb{E}\left[\left(\mathfrak{c}_{\mathbb{r}}^{-1} e^{-\xi h_{\mathbb{r}}(z)} D_{h}\left(B_{\varepsilon \mathbb{r}}(z), \partial B_{2 \varepsilon \mathbb{r}}(z)\right)\right)^{-p}\right]=\varepsilon^{-\xi Q p-\xi^{2} p^{2} / 2+o_{\varepsilon}(1)},
$$

uniformly over all $z \in \mathbb{C}$ and $\mathbb{r}>0$. Applying the Chebyshev inequality and setting $p=(s-\xi Q) / \xi^{2}$ gives (3.68) with $h_{\mathbb{r}}(z)$ in place of $h_{\mathbb{r}}(0)$. For $z \in \mathbb{r} K$, we can replace $h_{\mathrm{r}}(z)$ with $h_{\mathrm{r}}(0)$ via exactly the same argument as in the proof of Lemma 3.19.

Lemma 3.22 For each $\chi^{\prime}>\xi(Q+2)$ and each $\mathbb{r}>0$, it holds with polynomially high probability as $\varepsilon \rightarrow 0$, at a rate which is uniform in $\mathbb{r}$, that

$$
\mathfrak{c}_{\mathbb{r}}^{-1} e^{-\xi h_{\mathbb{r}}(0)} D_{h}(u, v) \geq\left|\frac{u-v}{\mathbb{r}}\right|^{\chi^{\prime}}, \quad \forall u, v \in K \text { with }|u-v| \leq \varepsilon .
$$


Proof This follows from (3.62), applied with $s=\chi^{\prime}$ and with $2^{-k} \varepsilon$ for $k \in \mathbb{N}_{0}$ in place of $\varepsilon$, together with a union bound over all $z \in B_{\varepsilon \mathrm{r}}(K) \cap\left(2^{-k-2} \varepsilon \mathrm{r} \mathbb{Z}^{2}\right)$ and then over all $k \in \mathbb{N}_{0}$.

Proof of Proposition 3.18 Combine Lemmas 3.20 and 3.22.

To conclude the proof of Theorem 1.7, we need to check that the Hölder exponents $\xi(Q-2)$ and $(\xi(Q+2))^{-1}$ are optimal.

Lemma 3.23 Let $V \subset \mathbb{C}$ be an open set. Almost surely, the identity map from $V$, equipped with the Euclidean metric, to $\left(V,\left.D_{h}\right|_{V}\right)$ is not Hölder continuous with any exponent greater than $\xi(Q-2)$. Furthermore, the inverse of this map is not Hölder continuous with any exponent greater than $\xi^{-1}(Q+2)^{-1}$.

Proof The idea of the proof is to use Proposition 3.14 to study $D_{h}$-distances as we approach an $\alpha$-thick point of $h$ for $\alpha$ close to 2 or to -2 . To produce such a thick point, we will sample a point from the $\alpha$-LQG measure induced by the zero-boundary part of $\left.h\right|_{V}$. By Axiom III, we can assume without loss of generality that $h$ is normalized so that $h_{1}(0)=0$. We can also assume without loss of generality that $V$ is bounded with smooth boundary. Let $h^{V}$ be the zero-boundary part of $\left.h\right|_{V}$, so that $h-h^{V}$ is harmonic on $V$.

Let $\alpha \in(-2,2)$ which we will eventually send to either -2 or 2 , and let $\mu_{h^{V}}^{\alpha}$ be the $\alpha$-LQG measure induced by $h^{V}$. Also let $\mathbb{Z}$ be sampled uniformly from $\mu_{h}^{\alpha}$, normalized to be a probability measure. Let $\widetilde{\mathbb{P}}$ be the law of $(h, \mathbb{Z})$ weighted by the total mass $\mu_{h^{V}}^{\alpha}(V)$, so that under $\widetilde{\mathbb{P}}, h$ is sampled from its marginal law weighted by $\mu_{h^{V}}^{\alpha}(V)$ and conditional on $h, \mathbb{Z}$ is sampled from $\mu_{h^{V}}^{\alpha}$, normalized to be a probability measure. By a well-known property of the $\alpha$-LQG measure (see, e.g., [15, Lemma A.10]), a sample $(h, \mathbb{Z})$ from the law $\widetilde{\mathbb{P}}$ can be equivalently be produced by first sampling $\widetilde{h}$ from the unweighted marginal law of $h$, then independently sampling $\mathbb{Z}$ uniformly from Lebesgue measure on $\mathbb{S}^{\prime}$ and setting $h=\widetilde{h}-\alpha \log |\cdot-\mathbb{Z}|+\mathfrak{g}_{\mathbb{Z}}$, where $\mathfrak{g}_{\mathbb{Z}}: V \rightarrow \mathbb{R}$ is a deterministic continuous function.

By Proposition 3.14 (applied with the field $\widetilde{h}-\alpha \log |\cdot-\mathbb{Z}|$ in place of $h^{\alpha}$ ), the fact that $\mathfrak{g}_{\mathbb{Z}}$ is a.s. bounded in a neighborhood of $\mathbb{Z}$ (by continuity), and the Borel-Cantelli lemma, we find that a.s.

$$
D_{h}\left(\mathbb{Z}, \partial B_{r}(\mathbb{Z})\right)=r^{o_{r}(1)} \frac{c_{r}}{r^{\alpha \xi}} \int_{0}^{\infty} e^{\xi \widetilde{h}_{r e^{-t}(\mathbb{Z})-\xi(Q-\alpha) t o_{t}(t)}} d t
$$

where here the $o_{t}(t)$ is deterministic and tends to 0 as $t \rightarrow \infty$ (it comes from the error $\psi(t)$ in Proposition 3.14) and the $o_{r}(1)$ denotes a random variable which tends to 0 a.s. as $r \rightarrow 0$. The description in the preceding paragraph shows that conditional on $\mathbb{Z}$, the process $t \mapsto \widetilde{h}_{r e^{-t}}(\mathbb{Z})-\widetilde{h}_{r}(\mathbb{Z})$ evolves as a standard linear Brownian motion. Consequently, the Gaussian tail bound shows that with probability tending to 1 as $r \rightarrow 0$,

$$
\int_{0}^{\infty} e^{\xi \widetilde{h}_{r e^{-t}(\mathbb{Z})-\xi(Q-\alpha) t+o_{t}(t)}} d t=r^{o_{r}(1)} e^{\xi \widetilde{h}_{r}(\mathbb{Z})}=r^{o_{r}(1)} .
$$


By plugging (3.72) into (3.71) and using the fact that $\mathfrak{c}_{r}=r^{\xi Q+o_{r}(1)}$ (Theorem 1.5), it therefore follows that with probability tending to 1 as $r \rightarrow 0$,

$$
D_{h}\left(\mathbb{Z}, \partial B_{r}(\mathbb{Z})\right)=r^{\xi(Q-\alpha)+o_{r}(1)} .
$$

Since $\alpha$ can be made arbitrarily close to 2, this shows the desired lack of Hölder continuity for identity map $(V,|\cdot|) \rightarrow\left(V, D_{h}\right)$. Since $\alpha$ can be made arbitrarily close to -2 , we also get the desired lack of Hölder continuity for the inverse map $\left(V, D_{h}\right) \rightarrow(V,|\cdot|)$.

\section{Constraints on the behavior of $D_{h}$-geodesics}

Let $D$ be a weak $\gamma$-LQG metric. By Lemma 3.8, for a whole-plane GFF $h$, the metric space $\left(\mathbb{C}, D_{h}\right)$ is a boundedly compact length space (i.e., closed bounded subsets are compact) so there is a $D_{h}$-geodesic-i.e., a path of minimal $D_{h}$-length—between any two points of $\mathbb{C}$ [5, Corollary 2.5.20]. In this section we will apply the main results of this paper to prove two estimates which constrain the behavior of $D_{h}$-geodesics. The first of these estimates, Proposition 4.1, tells us that paths which stay in a small Euclidean neighborhood of a straight line or an arc of the boundary of a circle have large $D_{h}$-lengths. In particular, $D_{h}$-geodesics are unlikely to stay in such a neighborhood. The second estimate, Proposition 4.3, says that a $D_{h}$-geodesic cannot spend a long time near the boundary of a $D_{h}$-metric ball.

\subsection{Lower bound for $D_{h}$-distances in a narrow tube}

Proposition 4.1 Let $L \subset \mathbb{C}$ be a compact set which is either a line segment, an arc of a circle, or a whole circle and fix $b>0$. For each $\mathrm{r}>0$ and each $p>0$, it holds with probability at least $1-\varepsilon^{p^{2} /\left(2 \xi^{2}\right)+o_{\varepsilon}(1)}$ that

$$
\inf \left\{D_{h}\left(u, v ; B_{\varepsilon \mathbb{r}}(\mathbb{r} L)\right): u, v \in B_{\varepsilon \mathbb{r}}(\mathbb{r} L),|u-v| \geq b \mathbb{r}\right\} \geq \varepsilon^{p+\xi Q-1-\xi^{2} / 2} \mathfrak{c}_{\mathbb{r}} e^{\xi h_{\mathbb{r}}(0)},
$$

where the rate of the $o_{\varepsilon}(1)$ depends on $L, b, p$ but not on $\mathbb{r}$.

By [2, Theorem 1.9], for each $\gamma \in(0,2)$ we have $\xi Q \leq 1$ and hence $\xi Q-1-$ $\xi^{2} / 2<0$. Therefore, the power of $\varepsilon$ on the right side of (4.1) is negative for small enough $p$. Hence, Proposition 4.1 implies that when $\varepsilon$ is small and $u, v \in B_{\varepsilon \mathbb{r}}(\mathbb{r} L)$ with $|u-v| \geq b \mathbb{r}$, it holds with high probability that $D_{h}\left(u, v ; B_{\varepsilon \mathbb{r}}(\mathbb{r} L)\right)$ is much larger than $D_{h}(u, v)$. In particular, a $D_{h}$-geodesic from $u$ to $v$ cannot stay in $B_{\varepsilon \mathbb{r}}(L)$.

Proof of Proposition 4.1 Step 1: Bounding distances in terms of circle averages. View L as a path $[0,|L|] \rightarrow \mathbb{C}$ parametrized by Euclidean unit speed. For $k \in[0,|L| /(6 \varepsilon)]_{\mathbb{Z}}$, let $z_{k}^{\varepsilon}:=\mathrm{r} L(6 k \varepsilon)$. Then the balls $B_{3 \varepsilon \mathrm{r}}\left(z_{k}^{\varepsilon}\right)$ are disjoint and the balls $B_{7 \varepsilon \mathrm{r}}\left(z_{k}^{\varepsilon}\right)$ cover $B_{\varepsilon \mathrm{r}}(\mathrm{r} L)$. 
Fix $\zeta \in(0,1)$, which we will eventually send to zero. By Proposition 3.1 and a union bound, it holds with superpolynomially high probability as $\varepsilon \rightarrow 0$ that

$$
D_{h}\left(B_{2 \varepsilon \mathbb{\Gamma}}\left(z_{k}^{\varepsilon}\right), B_{3 \varepsilon \mathbb{I}}\left(z_{k}^{\varepsilon}\right)\right) \geq \varepsilon^{\zeta} \mathfrak{c}_{\varepsilon \mathbb{r}} e^{\xi h_{\varepsilon \mathbb{r}}\left(z_{k}^{\varepsilon}\right)}, \quad \forall k \in[0,|L| /(6 \varepsilon)]_{\mathbb{Z}}
$$

Henceforth assume that (4.2) holds. The idea of the proof is that a path in $B_{\varepsilon \mathrm{r}}(\mathrm{r} L)$ has to cross between the inner and outer boundaries of a large number of the annuli $B_{3 \varepsilon \mathrm{r}}\left(z_{k}^{\varepsilon}\right) \backslash B_{2 \varepsilon \mathrm{r}}\left(z_{k}^{\varepsilon}\right)$. Thus (4.2) reduces our problem to proving a lower bound for the sum of the quantities $\varepsilon^{\zeta} \mathfrak{c}_{\varepsilon \mathrm{r}} e^{\xi h_{\varepsilon \mathrm{r}}}\left(z_{k}^{\varepsilon}\right)$ for these annuli, which in turn can be proven using Theorem 1.5 and basic estimates for the circle average process.

Step 2: Lower-bounding lengths of paths in $B_{\varepsilon \mathrm{r}}(\mathrm{r} L)$ in terms of circle averages. There is a constant $c>0$ depending only on $b$ and $L$ such that for small enough $\varepsilon>0$ (depending only on $b$ and $L$ ), the following is true. If $u, v \in B_{\varepsilon \mathrm{r}}(\mathrm{r} L)$ satisfy $|u-v| \geq b \mathrm{r}$, there are integers $0 \leq k_{1}^{\prime}<k_{2}^{\prime} \leq|L| /(6 \varepsilon)$ such that $k_{2}^{\prime}-k_{1}^{\prime} \geq c \varepsilon^{-1}$, $u \in B_{7 \varepsilon \mathrm{r}}\left(z_{k_{1}^{\prime}}^{\varepsilon}\right)$, and $v \in B_{7 \varepsilon \mathrm{r}}\left(z_{k_{2}^{\prime}}^{\varepsilon}\right)$. Each path from $u$ to $v$ in $B_{\varepsilon \mathrm{r}}(\mathrm{r} L)$ must enter $B_{2 \varepsilon \mathrm{r}}\left(z_{k}^{\varepsilon}\right)$ for each $k \in\left[k_{1}^{\prime}+2, k_{2}^{\prime}-2\right]_{\mathbb{Z}}$, and hence must cross the annulus $\mathbb{A}_{2 \varepsilon \mathrm{r}, 3 \varepsilon \mathrm{r}}\left(z_{k}^{\varepsilon}\right)$ for each such $k$. Combining this with (4.2) shows that

$$
D_{h}\left(u, v ; B_{\varepsilon \mathbb{r}}(\mathbb{r} L)\right) \geq \varepsilon^{\zeta} \mathfrak{c}_{\varepsilon \mathbb{r}} \sum_{k=k_{1}^{\prime}+2}^{k_{2}^{\prime}-2} e^{\xi h_{\varepsilon \mathbb{r}}\left(z_{k}^{\varepsilon}\right)}
$$

Step 3: Proof conditional on a circle average estimate. We claim that for any fixed $k_{1}, k_{2} \in[0,|L| /(6 \varepsilon)]_{\mathbb{Z}}$ with $k_{2}-k_{1} \geq(c / 2) \varepsilon^{-1}$ and any $p>0$,

$$
\mathbb{P}\left[\sum_{k=k_{1}}^{k_{2}} e^{\xi h_{\varepsilon \mathbb{I}}\left(z_{k}^{\varepsilon}\right)} \geq \varepsilon^{p-1-\xi^{2} / 2} e^{\xi h_{\varepsilon \mathbb{I}}(0)}\right] \geq 1-\varepsilon^{\frac{p^{2}}{2 \xi^{2}}+o_{\varepsilon}(1)}
$$

where the rate of the $o_{\varepsilon}(1)$ depends on $L, b, p$ but not on $\mathbb{r}$ or the particular choice of $k_{1}, k_{2}$. We will prove (4.4) just below using standard Gaussian estimates.

Let us first conclude the proof assuming (4.4). We can find a constant-order number of pairs $k_{1}, k_{2} \in[0,|L| /(6 \varepsilon)]_{\mathbb{Z}}$ with $k_{2}-k_{1} \geq(c / 2) \varepsilon^{-1}$ such that for small enough $\varepsilon$ (depending only on $L$ and $b$ ), each interval $\left[k_{1}^{\prime}+2, k_{2}^{\prime}-2\right] \subset[0,|L| /(6 \varepsilon)]_{\mathbb{Z}}$ with $\left|k_{2}^{\prime}-k_{1}^{\prime}\right| \geq c \varepsilon^{-1}$ contains one of the intervals $\left[k_{1}, k_{2}\right]$.

By applying (4.4) (with $p-2 \zeta$ in place of $p$ ) to each such pair $k_{1}, k_{2}$, then taking a union bound, we get that with probability at least $1-\varepsilon^{\frac{(p-2 \zeta)^{2}}{2 \xi^{2}}+o_{\varepsilon}(1)}$, the sum on the right side of (4.3) is bounded below by $\varepsilon^{p-1-\xi^{2} / 2-2 \zeta} e^{\xi h_{\varepsilon \mathrm{r}}(0)}$ simultaneously for every possible choice of $k_{1}^{\prime}, k_{2}^{\prime}$. By (4.3), with probability at least $1-\varepsilon^{\frac{(p-2 \zeta)^{2}}{2 \xi^{2}}+o_{\varepsilon}(1)}$ it holds simultaneously for each $u, v \in B_{\varepsilon \mathbb{r}}(\mathbb{r} L)$ satisfying $|u-v| \geq b \mathbb{r}$ that

$$
D_{h}\left(u, v ; B_{\varepsilon \mathbb{r}}(\mathbb{r} L)\right) \geq \varepsilon^{p-1-\xi^{2} / 2-\zeta} \mathfrak{c}_{\varepsilon \mathbb{r}} e^{\xi h_{\mathbb{T}}(0)} \geq \varepsilon^{p+\xi Q-1-\xi^{2} / 2-\zeta+o_{\varepsilon}(1)} \mathfrak{c}_{\mathbb{r}} e^{\xi h_{\mathbb{r}}(0)}
$$


where in the second inequality we use Theorem 1.5. Sending $\zeta \rightarrow 0$ now gives (4.1). Step 4: Proof of the circle average estimate. The rest of the proof is devoted to proving the inequality (4.4). To lighten notation, write $X_{k}:=h_{\varepsilon \mathbb{r}}\left(z_{k}^{\varepsilon}\right)-h_{\mathbb{r}}(0)$. By the calculations in [16, Section 3.1] (and the scale invariance of the law of $h$, modulo additive constant), the $X_{k}$ 's are jointly centered Gaussian with variances satisfying

$$
\operatorname{Var}\left(X_{k}\right)=\log \varepsilon^{-1}+O(1)
$$

where here $O(1)$ denotes a quantity which is bounded above and below by constants depending only on $L, b$ (not on $\varepsilon, \mathbb{r}, j, k$ ). Since $z_{k}^{\varepsilon}=\mathbb{r} L(6 k \varepsilon)$ and $L$ is parametrized by Euclidean unit speed, we also have the following covariance formula for $j \neq k$ :

$$
\operatorname{Cov}\left(X_{j}, X_{k}\right)=\log \left(\frac{\mathbb{r}}{\left|z_{j}^{\varepsilon}-z_{k}^{\varepsilon}\right|}\right)+O(1)=\log \left(\frac{1}{\varepsilon|k-j|}\right)+O(1) .
$$

Recall the formula $\mathbb{E}\left[e^{X}\right]=e^{\operatorname{Var}(X) / 2}$ for a centered Gaussian random variable $X$. Applying this to the $X_{k}$ 's and recalling (4.6) and the fact that $k_{2}-k_{1} \asymp \varepsilon^{-1}$ gives

$$
\mathbb{E}\left[\sum_{k=k_{1}}^{k_{2}} e^{\xi X_{k}}\right] \asymp \varepsilon^{-1-\xi^{2} / 2}
$$

with the implicit constant depending only on $L, b$. From (4.6) and (4.7) we obtain $\operatorname{Var}\left(X_{j}+X_{k}\right)=\log \left(\varepsilon^{-4}|k-j|^{-2}\right)+O(1)$ for $j \neq k$. Hence

$$
\begin{aligned}
\mathbb{E}\left[\left(\sum_{k=k_{1}}^{k_{2}} e^{\xi X_{k}}\right)^{2}\right] & =\sum_{k=k_{1}}^{k_{2}} \mathbb{E}\left[e^{2 \xi X_{k}}\right]+2 \sum_{k=k_{1}}^{k_{2}} \sum_{j=k+1}^{k_{2}} \mathbb{E}\left[e^{\xi\left(X_{j}+X_{k}\right)}\right] \\
& \preceq \varepsilon^{-1-2 \xi^{2}}+2 \varepsilon^{-2 \xi^{2}} \sum_{k=k_{1}}^{k_{2}} \sum_{j=k+1}^{k_{2}}|j-k|^{-\xi^{2}} \\
& \preceq \varepsilon^{-1-2 \xi^{2}}+\varepsilon^{-2-\xi^{2}} \preceq \varepsilon^{-2-\xi^{2}}
\end{aligned}
$$

with the implicit constants depending only on $L, b$, where in the last inequality we use that $\xi<2 / d_{2}<1$, so $1+2 \xi^{2}<2+\xi^{2}$.

By (4.8), (4.9), and the Payley-Zygmund inequality, we find that there is a constant $a=a(L)>0$ such that

$$
\mathbb{P}\left[\sum_{k=k_{1}}^{k_{2}} e^{\xi X_{k}} \geq a \varepsilon^{-1-\xi^{2} / 2}\right] \geq a
$$

To improve the lower bound for this probability, we will apply the following elementary Gaussian concentration bound (see, e.g., [18, Lemma 2.1]): 
Lemma 4.2 For any $a>0$, there exists $C=C(a)>0$ such that the following is true. Let $\mathbf{X}=\left(X_{1}, \ldots, X_{n}\right)$ be a centered Gaussian vector taking values in $\mathbb{R}^{n}$ and let $\sigma^{2}:=\max _{1 \leq j \leq n} \operatorname{Var}\left(X_{j}\right)$. If $B \subset \mathbb{R}^{n}$ such that $\mathbb{P}[X \in B] \geq a$, then for any $\lambda \geq C \sigma$,

$$
\mathbb{P}\left[\inf _{\mathbf{x} \in B}|\mathbf{X}-\mathbf{x}|_{\infty}>\lambda\right] \leq e^{-\frac{(\lambda-C \sigma)^{2}}{2 \sigma^{2}}}
$$

where $|\cdot|_{\infty}$ is the $L^{\infty}$ norm on $\mathbb{R}^{n}$.

We now apply Lemma 4.2 with $a$ as in (4.10), with $\sigma^{2}=\log \varepsilon^{-1}+O(1)$ [recall (4.6)], with

$$
B=\left\{\left(x_{k_{1}}, \ldots, x_{k_{2}}\right) \in \mathbb{R}^{k_{1}+k_{2}+1}: \sum_{k=k_{1}}^{k_{2}} e^{\xi x_{k}} \geq a \varepsilon^{-1-\xi^{2} / 2}\right\}
$$

and with $\lambda=\frac{p}{\xi} \log \varepsilon^{-1}$. This shows that with probability $1-\varepsilon^{p^{2} /\left(2 \xi^{2}\right)+o_{\varepsilon}(1)}$, there exists $\left(x_{k_{1}}, \ldots, x_{k_{2}}\right) \in B$ such that $\max _{k \in\left[k_{1}, k_{2}\right]_{\mathbb{Z}}}\left|X_{k}-x_{k}\right| \leq \frac{p}{\xi} \log \varepsilon^{-1}$. If this is the case, then

$$
\sum_{k=k_{1}}^{k_{2}} e^{\xi X_{k}} \geq \varepsilon^{p} \sum_{k=k_{1}}^{k_{2}} e^{\xi x_{k}} \geq a \varepsilon^{p-1-\xi^{2} / 2}
$$

Since $X_{k}=h_{\varepsilon \mathbb{r}}\left(z_{k}^{\varepsilon}\right)-h_{\mathbb{r}}(0)$, this implies (4.4).

\section{2 $D_{h}$-geodesics cannot trace the boundaries of $D_{h}$-metric balls}

For $s>0$ and $z \in \mathbb{C}$, we write $\mathcal{B}_{s}\left(z ; D_{h}\right)$ for the $D_{h}$-metric ball of radius $s$ centered at $z$. The following proposition prevents a $D_{h}$-geodesic from spending a long time near the boundary of a $D_{h}$-metric ball.

Proposition 4.3 For each $M>0$ and each $\mathrm{r}>0$, it holds with superpolynomially high probability as $\varepsilon \rightarrow 0$, at a rate which is uniform in the choice of $\mathbb{r}$, that the following is true. For each $s>0$ for which $\mathcal{B}_{s}\left(0 ; D_{h}\right) \subset B_{\varepsilon^{-M_{\mathrm{r}}}}(0)$ and each $D_{h}$-geodesic $P$ from 0 to a point outside of $\mathcal{B}_{s}\left(0 ; D_{h}\right)$,

$$
\operatorname{area}\left(B_{\varepsilon \mathbb{r}}(P) \cap B_{\varepsilon \mathbb{r}}\left(\partial \mathcal{B}_{s}\left(0 ; D_{h}\right)\right)\right) \leq \varepsilon^{2-1 / M_{\mathbb{P}^{2}}},
$$

where area denotes 2-dimensional Lebesgue measure.

For $C>1, z \in \mathbb{C}$, and $r>0$, we say that the Euclidean ball $B_{r}(z)$ is $C$-good if

$$
\sup _{u, v \in \partial B_{r}(z)} D_{h}\left(u, v ; \mathbb{A}_{r / 2,2 r}(z)\right) \leq C D_{h}\left(\partial B_{r}(z), \partial B_{2 r}(z)\right)
$$




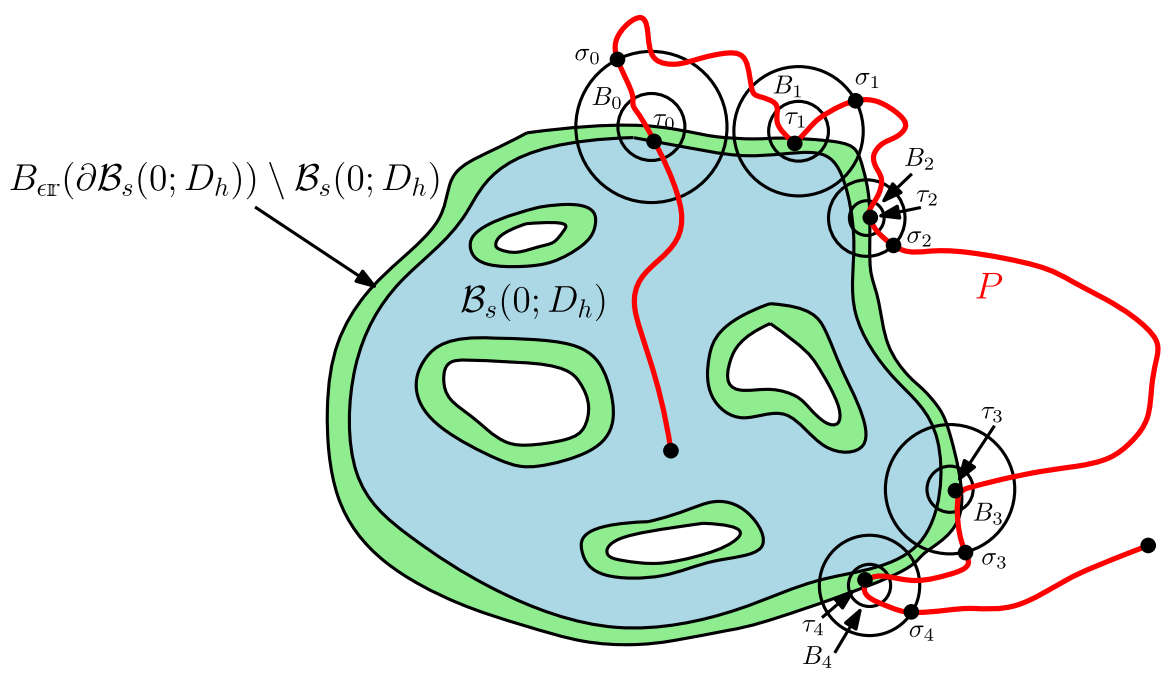

Fig. 5 Illustration of the proof of Proposition 4.3. By considering successive times at which $P$ enters $B_{\varepsilon \mathrm{r}}\left(\mathcal{B}_{S}\left(0 ; D_{h}\right)\right)$, we can find $K \in \mathbb{N}$ and a collection of $K C$-good Euclidean balls $B_{0}, \ldots, B_{K}$ with radii in $\left[2 \varepsilon \mathrm{r}, \varepsilon^{1-\zeta}\right.$ r] with the following properties: (a) each $B_{k}$ intersects $\partial \mathcal{B}_{S}\left(0 ; D_{h}\right)$; (b) the $D_{h}$-geodesic $P$ crosses the annuli $\left(2 B_{k}\right) \backslash B_{k}$ for $k \in[0, K-1]_{\mathbb{Z}}$ in numerical order; and (c) the balls of radii $4 \varepsilon^{1-\zeta_{\mathrm{r}}}$ with the same centers as the $B_{k}$ 's cover $P \cap B_{\varepsilon \mathrm{r}}\left(\mathcal{B}_{S}\left(0 ; D_{h}\right)\right)$. This last property implies that area $\left(B_{\varepsilon \mathrm{r}}(P) \cap B_{\varepsilon \mathrm{r}}\left(\partial \mathcal{B}_{S}\left(0 ; D_{h}\right)\right) \leq\right.$ const $\times \varepsilon^{2-2 \zeta} \mathrm{r}^{2} K$, so we are left to bound $K$. To this end, we show using the definition (4.15) of a $C$-good ball and the fact that $P$ is a $D_{h}$-geodesic that $D_{h}\left(\partial B_{k}, \partial\left(2 B_{k}\right)\right)$ increases exponentially in $k$. Due to Lemma 4.5, this implies that $K \leq \varepsilon^{-1 /(2 M)}$

To prove Proposition 4.3, we will consider $C$-good balls which intersect $\partial \mathcal{B}_{s}\left(0 ; D_{h}\right)$ and which are hit by a given $D_{h}$-geodesic started from 0 . See Fig. 5 for an illustration and outline of the proof.

Lemma 4.4 For each $\zeta \in(0,1)$ and each $M>0$, there exists $C=C(\zeta, M)>1$ such that for each $\mathrm{r}>0$, it holds with probability at least $1-O_{\varepsilon}\left(\varepsilon^{M}\right)$, at a rate which is uniform in $\mathbb{r}$, that the Euclidean ball $B_{\varepsilon^{-M_{\mathbb{r}}}}(0)$ can be covered by $C$-good balls with radii in $\left[2 \varepsilon \mathbb{r}, \varepsilon^{1-\zeta} \mathbb{r}\right]$.

Proof This is an immediate consequence of Lemma 3.2 applied with $\varepsilon^{1-\zeta}$ in place of $\varepsilon$ and any choice of $v \in\left(0, \frac{1}{1-\zeta}-1\right)$.

We will also need the following easy consequence of the distance bounds from Sect. 3 .

Lemma 4.5 For each $M>0$, there exists $A=A(M)>0$ such that for each $\mathbb{r}>0$, the following holds with probability $1-O_{\varepsilon}\left(\varepsilon^{M}\right)$ as $\varepsilon \rightarrow 0$, at a rate which is uniform in $\mathbb{r}$. For each $z, w \in B_{\varepsilon^{-} M_{\mathbb{r}}}(0)$ with $|z-w| \geq \varepsilon \mathbb{r}$,

$$
D_{h}(z, w) \geq \varepsilon^{A} \sup _{u, v \in B_{\varepsilon}-M_{\mathbb{R}}(0)} D_{h}(u, v) .
$$


Proof We will prove a lower bound for the left side of (4.16) [see (4.20)] and an upper bound for the right side of (4.16) [see (4.22)], then compare them.

By Proposition 3.1 and a union bound, it holds with superpolynomially high probability as $\varepsilon \rightarrow 0$ that

$$
D_{h}\left(\partial B_{\varepsilon \mathbb{r} / 4}(x), \partial B_{\varepsilon \mathbb{r} / 2}(x)\right) \geq \varepsilon \mathfrak{c}_{\varepsilon \mathbb{r}} e^{\xi h_{\varepsilon \mathbb{r}}(x)}, \quad \forall x \in B_{\varepsilon^{-M_{\mathbb{r}}}}(0) \cap\left(\frac{\varepsilon \mathbb{r}}{8} \mathbb{Z}^{2}\right)
$$

The circle averages $h_{\varepsilon \mathrm{r}}(x)-h_{\mathrm{r}}(0)$ for $x \in B_{\varepsilon^{-M_{\mathrm{r}}}}(0)$ are Gaussian with variance at most $(M+1) \log \varepsilon^{-1}$. By the Gaussian tail bound and a union bound, if we choose $A_{0}=A_{0}(M)$ to be sufficiently large, then it holds with probability $1-O_{\varepsilon}\left(\varepsilon^{M}\right)$ that

$$
\left|h_{\varepsilon \mathbb{r}}(x)-h_{\mathbb{r}}(0)\right| \leq A_{0} \log \varepsilon^{-1} \forall x \in B_{\varepsilon^{-M_{\mathbb{r}}}}(0) \cap\left(\frac{\varepsilon \mathbb{r}}{8} \mathbb{Z}^{2}\right)
$$

By Theorem 1.5,

$$
\mathfrak{c}_{\varepsilon \mathrm{r}}=\varepsilon^{\xi Q+o_{\varepsilon}(1)} \mathfrak{c}_{\mathbb{r}}
$$

If $z, w \in B_{\varepsilon^{-M_{\mathrm{r}}}}(0)$ with $|z-w| \geq \varepsilon \mathbb{r}$, then any path from $z$ to $w$ must cross between the inner and outer boundaries of an annulus of the form $B_{\varepsilon \mathrm{r} / 2}(x) \backslash B_{\varepsilon \mathrm{r} / 4}(x)$ for some $x \in B_{\varepsilon^{-M_{\mathbb{r}}}}(0) \cap\left(\frac{\varepsilon \mathbb{r}}{8} \mathbb{Z}^{2}\right)$. Combining this last observation with (4.17) shows that with superpolynomially high probability as $\varepsilon \rightarrow 0, D_{h}(z, w)$ is at least the right side of (4.17) for each such $z, w$. We then apply (4.18) and (4.19) to lower-bound the right side of (4.17). This shows that with probability $1-O_{\varepsilon}\left(\varepsilon^{M}\right)$,

$$
D_{h}(z, w) \geq \varepsilon^{\xi A_{0}+\xi Q+1+o_{\varepsilon}(1)} \mathfrak{c}_{\mathbb{r}} e^{\xi h_{\mathbb{r}}(0)}, \quad \forall z, w \in B_{\varepsilon^{-M_{\mathbb{T}}}}(0) \text { with }|z-w| \geq \varepsilon \mathbb{r} .
$$

By Proposition 3.9,

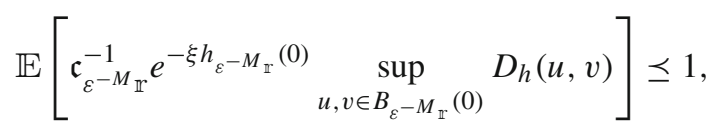

with the implicit constant uniform over all $\mathrm{r}>0$ and $\varepsilon \in(0,1)$. By Theorem 1.5, $\mathfrak{c}_{\varepsilon^{-M_{\mathrm{r}}}}=\varepsilon^{-\xi Q M+o_{\varepsilon}(1)} \mathfrak{c}_{\mathrm{r}}$. By the Gaussian tail bound, we can find $A_{1}=A_{1}(M)>0$ such that with probability $1-O_{\varepsilon}\left(\varepsilon^{M}\right)$, we have $\left|h_{\varepsilon^{-M_{\mathbb{T}}}}(0)-h_{\mathbb{r}}(0)\right| \leq A_{0} \log \varepsilon^{-1}$. Combining these estimates with (4.21) and Markov's inequality shows that with probability $1-O_{\varepsilon}\left(\varepsilon^{M}\right)$,

$$
\sup _{u, v \in B_{\varepsilon}-M_{\mathbb{T}}(0)} D_{h}(u, v) \leq \varepsilon^{-\xi A_{1}-\xi Q M-M+o_{\varepsilon}(1)} \mathfrak{c}_{\mathbb{r}} e^{\xi h_{\mathbb{I}}(0)} .
$$

Combining (4.20) and (4.22) gives (4.16) for any choice of $A>\xi A_{1}+\xi Q M+M+$ $\xi A_{0}+\xi Q+1$. 
Proof of Proposition 4.3 Step 1: Defining a regularity event. For $\tilde{M}>0, \zeta \in(0,1)$, $C>1$, and $A>1$, let $G_{\mathrm{r}}^{\varepsilon}=G_{\mathrm{r}}^{\varepsilon}(\tilde{M}, \zeta, C, A)$ be the event that the following is true.

1. The ball $B_{\varepsilon^{-} \widetilde{M}_{\mathbb{r}}}(0)$ can be covered by $C$-good Euclidean balls with radii in $\left[2 \varepsilon \mathbb{r}, \varepsilon^{1-\zeta} \mathbb{r}\right]$.

2. For each $z, w \in B_{\varepsilon^{-} \tilde{M}_{\mathbb{r}}}(0)$ with $|z-w| \geq \varepsilon \mathbb{r}$,

$$
D_{h}(z, w) \geq \varepsilon^{A} \sup _{u, v \in B_{\varepsilon^{-}-\widetilde{M}_{\mathbb{T}}}(0)} D_{h}(u, v) .
$$

By Lemmas 4.4 and 4.5 , for any $\widetilde{M}>0$ and $\zeta \in(0,1)$ we can find $C, A>1$ for which

$$
\mathbb{P}\left[G_{\mathbb{r}}^{\varepsilon}\right] \geq 1-O_{\varepsilon}\left(\varepsilon^{\tilde{M}}\right), \quad \text { uniformly over all } \mathbb{r}>0
$$

Henceforth assume that $G_{\mathrm{r}}^{\varepsilon}$ occurs for such a choice of $C, A$ and that $\tilde{M}>M$.

Step 2: Reducing to a bound for the number of excursions of a geodesic. Let $s>0$ such that $\mathcal{B}_{S}\left(0 ; D_{h}\right) \subset B_{\varepsilon^{-M_{\mathrm{r}}}}(0)$ and let $P$ be a $D_{h}$-geodesic from 0 to a point outside of $\mathcal{B}_{s}\left(0 ; D_{h}\right)$. Let $\tau_{0}=s$ and inductively for $k \in \mathbb{N}$ let $\tau_{k}$ be the first time $t$ after the exit time of $P$ from $B_{4 \varepsilon^{1-\zeta_{\mathrm{r}}}}\left(P\left(\tau_{k-1}\right)\right)$ for which $P(t) \in B_{\varepsilon \mathrm{r}}\left(\partial \mathcal{B}_{s}\right)$, or $\tau_{k}=\infty$ if no such time exists. Let $K$ be the smallest $k \in \mathbb{N}$ for which $\tau_{k}=\infty$.

We claim that there exists a constant $c>0$ depending on $C, A$ such that $K \leq c \log \varepsilon^{-1}$ on $G_{\mathrm{r}}^{\varepsilon}$. If this is the case, then $P \cap B_{\varepsilon \mathrm{r}}\left(\partial \mathcal{B}_{S}\right)$ can be covered by at most $c \log \varepsilon^{-1}$ Euclidean balls of radius $4 \varepsilon^{1-\zeta_{r}}$. This means that area $\left(B_{\varepsilon \mathrm{r}}(P) \cap B_{\varepsilon \mathrm{r}}\left(\partial \mathcal{B}_{S}\left(0 ; D_{h}\right)\right) \leq 4 \pi \varepsilon^{2-2 \zeta+o_{\varepsilon}(1)} \mathbb{r}^{2}\right.$. Choosing $\zeta<1 /(2 M)$ and sending $\widetilde{M} \rightarrow \infty$ then concludes the proof. Hence we only need to prove a logarithmic upper bound for $K$ assuming that $G_{\mathrm{r}}^{\varepsilon}$ occurs.

Step 3: bounding excursions using $C$-good balls. For $k \in[0, K-1]_{\mathbb{Z}}$, we can find a $C$-good Euclidean ball $B_{k}$ with radius in $\left[\varepsilon \mathbb{r}, \varepsilon^{1-\zeta} \mathbb{r}\right]$ which contains $P\left(\tau_{k}\right)$. Write $2 B_{k}$ for the Euclidean ball with the same center as $B_{k}$ and twice the radius of $B_{k}$. Let $\sigma_{k}$ be the first time after $\tau_{k}$ at which $P$ exits $2 B_{k}$. The time $\sigma_{k}$ is smaller than the exit time of $P$ from $B_{4 \varepsilon^{1-\zeta}}\left(P\left(\tau_{k}\right)\right)$. Consequently, the definition of the $\tau_{k}$ 's shows that $\sigma_{k} \in\left[\tau_{k}, \tau_{k+1}\right]$ for each $k \in[0, K]_{\mathbb{Z}}$.

Since $P$ is a $D_{h}$-geodesic and $P$ crosses the annulus $\left(2 B_{k}\right) \backslash B_{k}$ between times $\tau_{k}$ and $\sigma_{k}$,

$$
\sigma_{k}-\tau_{k} \geq D_{h}\left(\partial B_{k}, \partial\left(2 B_{k}\right)\right)
$$

We now argue that

$$
\tau_{k} \leq s+C D_{h}\left(\partial B_{k}, \partial\left(2 B_{k}\right)\right)
$$

Indeed, since $B_{k}$ intersects $B_{\varepsilon \mathrm{r}}\left(\partial \mathcal{B}_{s}\left(0 ; D_{h}\right)\right)$ and has radius at least $2 \varepsilon \mathrm{r}$, it follows that $B_{k}$ intersects $\partial \mathcal{B}_{s}\left(0 ; D_{h}\right)$. Let $z \in \partial \mathcal{B}_{s}\left(0 ; D_{h}\right)$ and let $t \in\left[\tau_{k}, \sigma_{k}\right]$ such that $P(t) \in \partial B_{k}$ (such a $t$ exists by the definition of $\sigma_{k}$ ). By the definition of a $C$-good 
ball, the $D_{h}$-diameter of $\partial B_{k}$ is at most $C D_{h}\left(\partial B_{k}, \partial\left(2 B_{k}\right)\right)$. Hence

$$
\tau_{k} \leq t \leq D_{h}(0, z)+D_{h}(z, P(t)) \leq s+C D_{h}\left(\partial B_{k}, \partial\left(2 B_{k}\right)\right)
$$

which is (4.26).

By (4.25) and (4.26) and the fact that the intervals $\left[\tau_{k}, \sigma_{k}\right] \subset[s, \infty)$ are disjoint, we get

$$
\sum_{j=0}^{k-1}\left(\sigma_{j}-\tau_{j}\right) \leq \tau_{k}-s \leq C\left(\sigma_{k}-\tau_{k}\right)
$$

This holds for each $k \in[0, K-1]_{\mathbb{Z}}$, from which we infer that

$$
\sigma_{K-1}-\tau_{K-1} \geq C^{-1}\left(1+C^{-1}\right)^{K}\left(\sigma_{0}-\tau_{0}\right)
$$

By the definition of $\sigma_{0}$, we have $\left|P\left(\sigma_{0}\right)-P\left(\tau_{0}\right)\right|=\varepsilon \mathrm{r}$. Moreover, since $P\left(\tau_{K-1}\right) \in$ $B_{\varepsilon \mathrm{r}}\left(B_{S}\left(0 ; D_{h}\right)\right), B_{S}\left(0 ; D_{h}\right) \subset B_{\varepsilon^{-M_{\mathrm{I}}}}(0)$, and $\widetilde{M}>M$, we have $P\left(\sigma_{K-1}\right), P\left(\tau_{K-1}\right) \in$ $B_{\varepsilon}-\widetilde{M}_{\mathbb{r}}(0)$. By (4.23) in the definition of $G_{\mathbb{r}}^{\varepsilon}$, it follows that

$$
\sigma_{0}-\tau_{0} \geq \varepsilon^{A}\left(\sigma_{K-1}-\tau_{K-1}\right) \text {. }
$$

Combining this with (4.27) shows that $C^{-1}\left(1+C^{-1}\right)^{K} \leq \varepsilon^{-A}$, which means that $K \leq \frac{A}{\log \left(1+C^{-1}\right)} \log \varepsilon^{-1}+O_{\varepsilon}(1)$, as required.

Acknowledgements We thank an anonymous referee for helpful comments on an earlier version of the manuscript. We thank Jian Ding, Alex Dunlap, Jason Miller, Scott Sheffield, as well as Vincent Tassion, Wendelin Werner, and their research group at ETH Zürich for helpful discussions. Part of the project was carried out during E. Gwynne and X. Sun's visit to MIT and E. Gwynne and J. Pfeffer's visit to Columbia University in Fall 2018. We thank the two institutions for their hospitality. J. Dubédat was partially supported by NSF Grant DMS-1512853. E. Gwynne was supported by a Herchel Smith fellowship and a Trinity College junior research fellowship. J. Pfeffer was partially supported by the National Science Foundation Graduate Research Fellowship under Grant No. 1122374. X. Sun was supported by the Simons Foundation as a Junior Fellow at Simons Society of Fellows, by NSF Grant DMS-1811092, and by the Minerva fund at the Department of Mathematics at Columbia University.

Open Access This article is licensed under a Creative Commons Attribution 4.0 International License, which permits use, sharing, adaptation, distribution and reproduction in any medium or format, as long as you give appropriate credit to the original author(s) and the source, provide a link to the Creative Commons licence, and indicate if changes were made. The images or other third party material in this article are included in the article's Creative Commons licence, unless indicated otherwise in a credit line to the material. If material is not included in the article's Creative Commons licence and your intended use is not permitted by statutory regulation or exceeds the permitted use, you will need to obtain permission directly from the copyright holder. To view a copy of this licence, visit http://creativecommons.org/licenses/by/4.0/.

\section{References}

1. Angel, O.: Growth and percolation on the uniform infinite planar triangulation. Geom. Funct. Anal. 13(5), 935-974 (2003). arXiv:math/0208123 
2. Ang, M.: Comparison of discrete and continuum Liouville first passage percolation (2019). ArXiv e-prints, arXiv:1904.09285

3. Ang, M., Park, M., Pfeffer, J., Sheffield, S.: Brownian loops and the central charge of a Liouville random surface (2020). ArXiv e-prints, arXiv:2005.11845

4. Adler, R.J., Taylor, J.E.: Random Fields and Geometry. Springer Monographs in Mathematics. Springer, New York (2007)

5. Burago, D., Burago, Y., Ivanov, S.: A Course in Metric Geometry. Graduate Studies in Mathematics, vol. 33. American Mathematical Society, Providence (2001)

6. Berestycki, N.: An elementary approach to Gaussian multiplicative chaos. Electron. Commun. Probab. 22, Paper No. 27, 12 (2017). arXiv:1506.09113

7. Bettinelli, J., Miermont, G.: Compact Brownian surfaces I: Brownian disks. Probab. Theory Relat. Fields 167(3-4), 555-614 (2017). arXiv:1507.08776

8. Borell, C.: The Brunn-Minkowski inequality in Gauss space. Invent. Math. 30(2), 207-216 (1975)

9. Ding, J., Dunlap, A.: Subsequential scaling limits for Liouville graph distance (2018). ArXiv e-prints, arXiv: 1812.06921

10. Ding, J., Dubédat, J., Dunlap, A., Falconet, H.: Tightness of Liouville first passage percolation for $\gamma \in(0,2)$ (2019). ArXiv e-prints, arXiv:1904.08021

11. Dubédat, J., Falconet, H.: Liouville metric of star-scale invariant fields: tails and Weyl scaling. Probab. Theory Relat. Fields (to appear) (2018). arXiv:1809.02607

12. Ding, J., Gwynne, E.: The fractal dimension of Liouville quantum gravity: universality, monotonicity, and bounds. Commun. Math. Phys. 374, 1877-1934 (2018). arXiv:1807.01072

13. Ding, J., Gwynne, E.: Tightness of supercritical Liouville first passage percolation (2020). ArXiv e-prints, arXiv:2005.13576

14. Ding, J., Gwynne, E., Sepúlveda, A.: The distance exponent for Liouville first passage percolation is positive (2020). ArXiv e-prints, arXiv:2005.13570

15. Duplantier, B., Miller, J., Sheffield, S.: Liouville quantum gravity as a mating of trees (2014). ArXiv e-prints, arXiv: 1409.7055

16. Duplantier, B., Sheffield, S.: Liouville quantum gravity and KPZ. Invent. Math. 185(2), 333-393 (2011). arXiv:1206.0212

17. Dufresne, D.: The distribution of a perpetuity, with applications to risk theory and pension funding. Scand. Actuar. J. 1-2, 39-79 (1990)

18. Ding, J., Zeitouni, O., Zhang, F.: Heat kernel for Liouville Brownian motion and Liouville graph distance. Commun. Math. Phys. (to appear) (2018). arXiv:1807.00422

19. Gwynne, E., Holden, N., Pfeffer, J., Remy, G.: Liouville quantum gravity with matter central charge in (1, 25): a probabilistic approach. Commun. Math. Phys. (to appear) (2019). arXiv:1903.09111

20. Gwynne, E., Holden, N., Sun, X.: A mating-of-trees approach for graph distances in random planar maps. Probab. Theory Relat. Fields (to appear) (2017). arXiv:1711.00723

21. Gwynne, E., Holden, N., Sun, X.: A distance exponent for Liouville quantum gravity. Probab. Theory Relat. Fields 173(3), 931-997 (2019). arXiv:1606.01214

22. Gwynne, E., Holden, N., Sun, X.: Mating of trees for random planar maps and Liouville quantum gravity: a survey (2019). ArXiv e-prints, arXiv:1910.04713

23. Gwynne, E., Miller, J.: Confluence of geodesics in Liouville quantum gravity for $\gamma \in(0,2)$. Ann. Probab. (to appear) (2019). arXiv:1905.00381

24. Gwynne, E., Miller, J.: Conformal covariance of the Liouville quantum gravity metric for $\gamma \in(0,2)$ (2019). ArXiv e-prints, arXiv:1905.00384

25. Gwynne, E., Miller, J.: Existence and uniqueness of the Liouville quantum gravity metric for $\gamma \in(0,2)$ (2019). ArXiv e-prints, arXiv: 1905.00383

26. Gwynne, E., Miller, J.: Local metrics of the Gaussian free field. Annales de l'Institut Fourier (to appear) (2019). arXiv:1905.00379

27. Gwynne, E., Miller, J., Sheffield, S.: The Tutte embedding of the mated-CRT map converges to Liouville quantum gravity (2017). ArXiv e-prints, arXiv:1705.11161

28. Gwynne, E., Miller, J., Sheffield, S.: Harmonic functions on mated-CRT maps. Electron. J. Probab. 24(58), 55 (2019). arXiv:1807.07511

29. Gwynne, E., Miller, J., Sheffield, S.: The Tutte embedding of the Poisson-Voronoi tessellation of the Brownian disk converges to $\sqrt{8 / 3}$-Liouville quantum gravity. Commun. Math. Phys. 374(2), 735-784 (2020). arXiv:1809.02091 
30. Gwynne, E., Pfeffer, J.: Bounds for distances and geodesic dimension in Liouville first passage percolation. Electron. Commun. Probab. 24(56), 12 (2019). arXiv:1903.09561

31. Gwynne, E., Pfeffer, J.: KPZ formulas for the Liouville quantum gravity metric. Trans. Am. Math. Soc. (to appear) (2019)

32. Hu, X., Miller, J., Peres, Y.: Thick points of the Gaussian free field. Ann. Probab. 38(2), 896-926 (2010). arXiv:0902.3842

33. Kahane, J.-P.: Sur le chaos multiplicatif. Ann. Sci. Math. Québec 9(2), 105-150 (1985)

34. Knizhnik, V., Polyakov, A., Zamolodchikov, A.: Fractal structure of 2D-quantum gravity. Mod. Phys. Lett. A 3(8), 819-826 (1988)

35. Le Gall, J.-F.: Uniqueness and universality of the Brownian map. Ann. Probab. 41(4), 2880-2960 (2013). arXiv:1105.4842

36. Miermont, G.: The Brownian map is the scaling limit of uniform random plane quadrangulations. Acta Math. 210(2), 319-401 (2013). arXiv:1104.1606

37. Miller, J., Qian, W.: The geodesics in Liouville quantum gravity are not Schramm-Loewner evolutions (2018). ArXiv e-prints, arXiv:1812.03913

38. Miller, J., Sheffield, S.: Liouville quantum gravity and the Brownian map II: geodesics and continuity of the embedding (2016). ArXiv e-prints, arXiv:1605.03563

39. Miller, J., Sheffield, S.: Liouville quantum gravity and the Brownian map III: the conformal structure is determined (2016). ArXiv e-prints, arXiv:1608.05391

40. Miller, J., Sheffield, S.: Imaginary geometry I: interacting SLEs. Probab. Theory Relat. Fields 164(3-4), 553-705 (2016)

41. Miller, J., Sheffield, S.: Quantum Loewner evolution. Duke Math. J. 165(17), 3241-3378 (2016). arXiv: 1312.5745

42. Miller, J., Sheffield, S.: Imaginary geometry IV: interior rays, whole-plane reversibility, and spacefilling trees. Probab. Theory Relat. Fields 169(3-4), 729-869 (2017). arXiv:1302.4738

43. Miller, J., Sheffield, S.: Liouville quantum gravity and the Brownian map I: the QLE $(8 / 3,0)$ metric. Invent. Math. 219(1), 75-152 (2020). arXiv:1507.00719

44. Rhodes, R., Vargas, V.: Gaussian multiplicative chaos and applications: a review. Probab. Surv. 11, 315-392 (2014). arXiv:1305.6221

45. Sudakov, V.N., Cirel' son, B.S.: Extremal properties of half-spaces for spherically invariant measures. Zap. Naučn. Sem. Leningrad. Otdel. Mat. Inst. Steklov. (LOMI), 41:14-24, 165, 1974. Problems in the theory of probability distributions, II

46. Sheffield, S.: Gaussian free fields for mathematicians. Probab. Theory Relat. Fields 139(3-4), 521-541 (2007). arXiv:math/0312099

47. Schramm, O., Sheffield, S.: A contour line of the continuum Gaussian free field. Probab. Theory Relat. Fields 157(1-2), 47-80 (2013). arXiv:math/0605337

48. Urbanik, K.: Functionals on transient stochastic processes with independent increments. Studia Math. 103(3), 299-315 (1992)

Publisher's Note Springer Nature remains neutral with regard to jurisdictional claims in published maps and institutional affiliations.

\section{Affiliations}

\section{Julien Dubédat ${ }^{1}$. Hugo Falconet ${ }^{1}$. Ewain Gwynne ${ }^{2}$ (D) - Joshua Pfeffer ${ }^{3}$. Xin Sun ${ }^{1}$}

Julien Dubédat

dubedat@math.columbia.edu

Hugo Falconet

hugo.falconet@columbia.edu

Joshua Pfeffer

pfeffer@mit.edu 
Xin Sun

xinsun@math.columbia.edu

1 Columbia University, New York, USA

2 University of Cambridge, Cambridge, UK

3 Massachusetts Institute of Technology, Cambridge, USA 